\title{
I-60442-0001
}

\author{
Calculation Package \\ for the \\ Analysis of Performance of Cells 1-6, with Underdrain, of the \\ Environmental Management Waste Management Facility \\ Oak Ridge, Tennessee
}

Date lssued: March 30, 2010

This docunent is approved for public release per review by: Arthu F. McBride $\quad$ Signature on File
$\begin{aligned} & \text { BJC ETTP CIassification \& } \\ & \text { Jnformation Office }\end{aligned}$ 


\section{Calculation Package \\ for the \\ Analysis of Performance of Cells 1-6, with Underdrain, of the Environmental Management Waste Management Facility, Oak Ridge, Tennessee}

Date Issued: March 30, 2010

Revision 0

Prepared by

Jacobs Enginetring Group Inc.

Oak Ridge, Tennessee

and

Portage, Inc.

Salt Lake City, Utah

Prepared for

the

U.S. Department of Energy

Office of Envionmental Management

BECHTEL JACOBS COMPANY LLC

managing the

Environmental Management Activities at the

East Tennessee Techrology Park

Y-12 National Security Complex Oak Ridge National Laboratory

under contract DE-AC05-980R22700

for the

U.S. DEPARTMENT OF ENERGY 


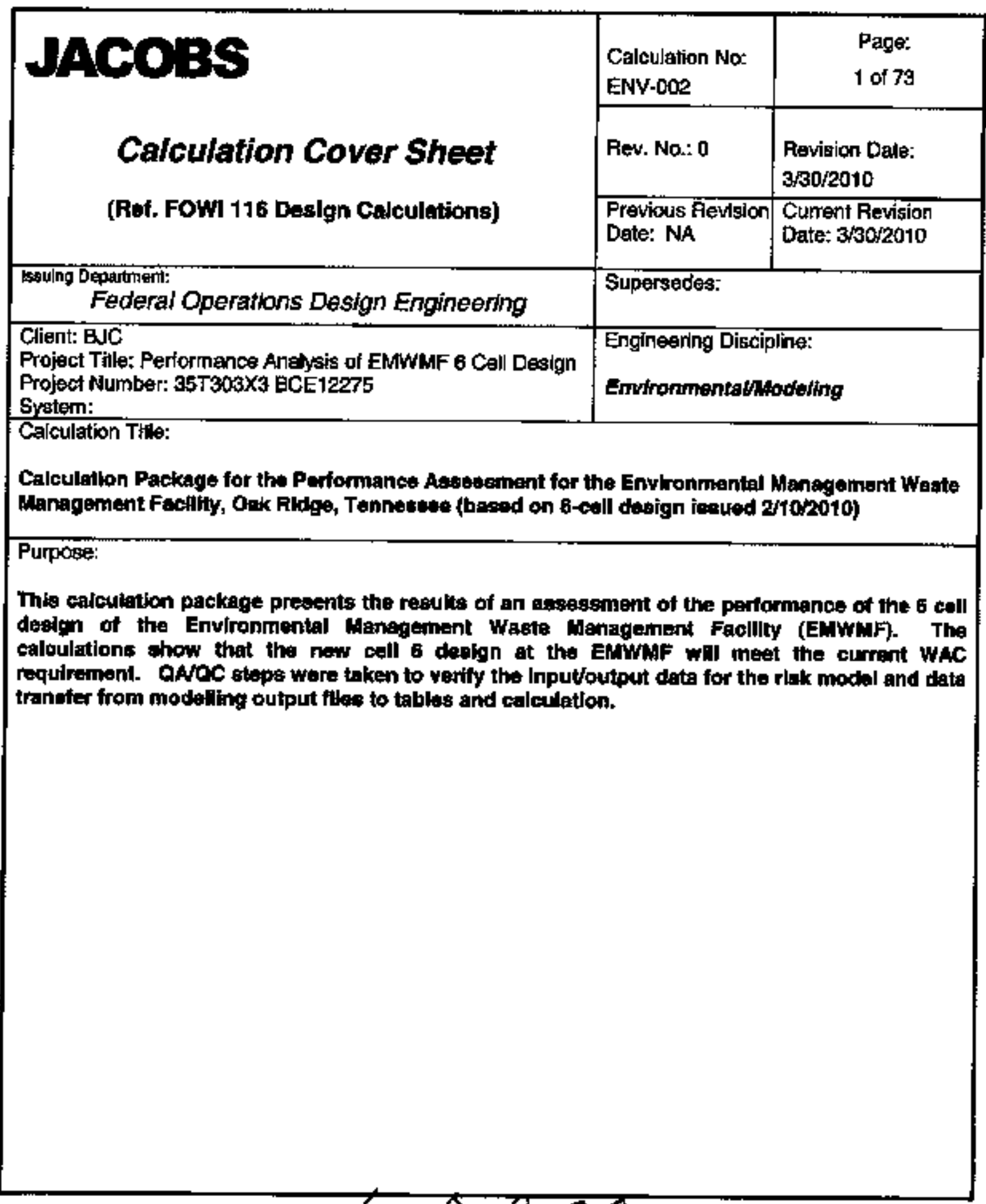

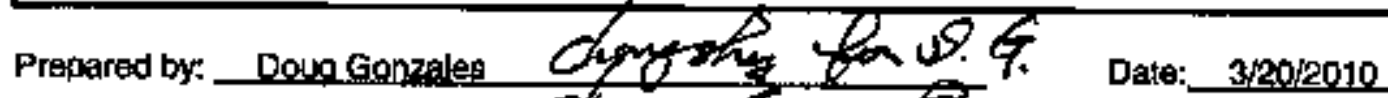

Checked by. Changsteng w Chine Date: 33012010

Engineering Manager's Approwat. Mark vohirso

Dale:_3/30/2010 


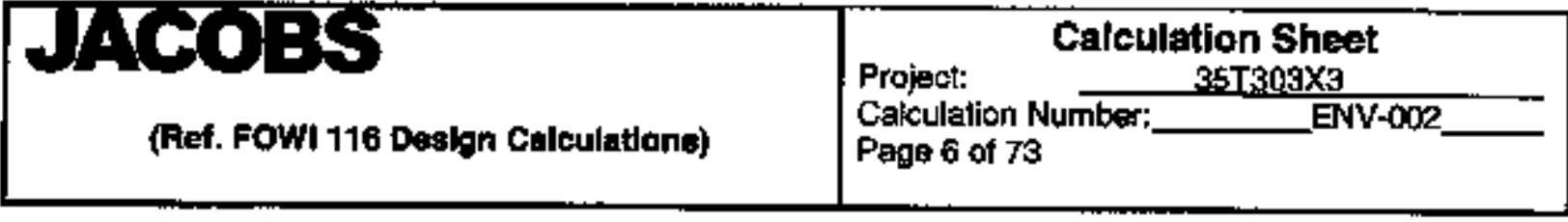

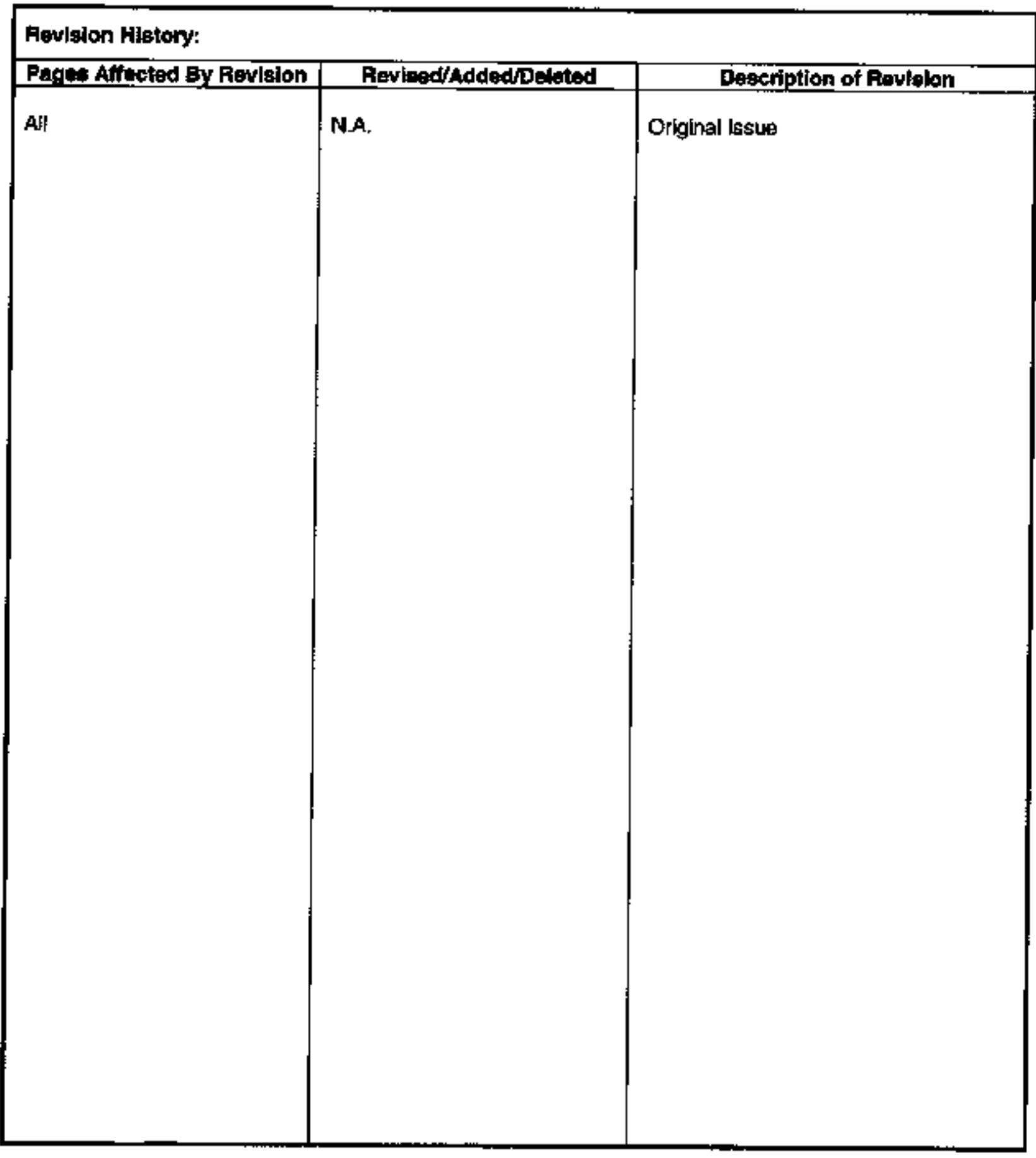




\begin{tabular}{|c|l|}
\hline $17.50: 53$ & $\begin{array}{l}\text { Calculation Sheet } \\
\text { Profect: } \\
\text { Calculation Number_35T303X3 } \\
\text { Page } 6 \text { of } 73\end{array}$ \\
\hline
\end{tabular}

Dencription of Caloulation:

Risk and dose calculation of the Cell 6 des gn at EMWMF and comparison to the current WAC.

\section{Aasumptions:}

Assumptions are as stated in the attachment. 
JACOBS

(Fef. FOW] 116 Design Calculations)

\section{Calculation Sheet}

Project: $\quad 355300 \times 3$

Calculation Number:__ ENvi-002

Page 6 of 73

Des:gn Inputs:

Dealgn Inpute are as noted In the Attachinent.

\begin{tabular}{|c|c|c|c|}
\hline Software: & & & \\
\hline Titlo & Devoloper & Versions & Revision Level \\
\hline $\begin{array}{c}\text { PATHRAE-RAD and } \\
\text { PATHRAE-HAZ }\end{array}$ & $\begin{array}{c}\text { Rogers and Associates } \\
\text { Engineering }\end{array}$ & 0 & 0 \\
\hline MODFLOW & McDonald and Harbaugh & 0 & 0 \\
\hline MODPATH & Pollock & 0 & 0 \\
\hline$M T 3 D$ & Žheng & 0 & 0 \\
\hline
\end{tabular}




\begin{tabular}{|c|c|}
\hline 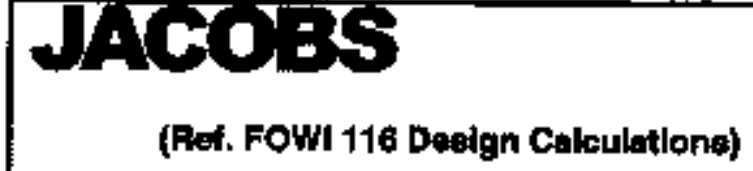 & $\begin{array}{l}\text { Calculation Sheet } \\
\text { Project: } \\
\text { Calculation Numbar: } \\
\text { Page } 6 \text { of } 73\end{array}$ \\
\hline
\end{tabular}

Coiculation Sentlon:

Ses Attachment. 


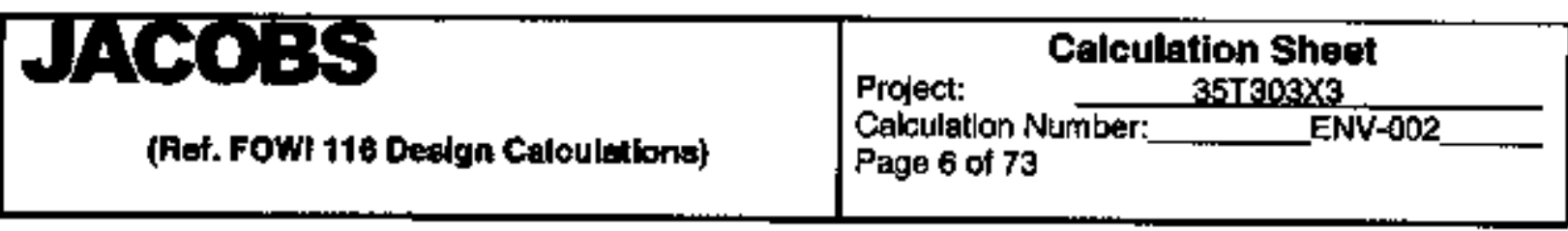

Conchutionthecommende|lons:

So: Attachment

fieference;

Sas Attachment 


\section{INTRODUCTION}

This document presents the results of an assessment of the performance of a build-out of the Environmental Management Waste Management Facility (EMWMF). The EMWMF configuration that was assessed includes the as-constructed Cells 1 through 4 , with a groundwater underdrain that was installed beneath Cell 3 during the winter of 2003-2004, and a Cell 5 that is presently under construction, and Cell 6, whose proposed design is addressed in an Addendum to Remedial Design Report for the Disposal of Oak Ridge Reservation Comprehensive Environmental Response, Compensation, and Liability Act of 1980 Waste, Oak Ridge, Tennessee, DOE/OR/O1-1873/VI\&D2/A6 (DOE 2010).

The total capacity of the EMWMF with six cells is about 2.2 million cubic yards. This assessment was conducted to determine the conditions under which the approved Waste Acceptance Criteria (WAC) for the EMWMF found in the Attainment Plan for Risk/ToxicityBased Waste Acceptance Criteria at the Oak Ridge Reservation, Oak Ridge, Tennessee (DOE 2001a), as revised for constituents added up to October 10, 2008 (Revised Table A.1: Analytic WAC Limits, http://bechteljacobs.org/webindex.h(ml\#3), would remain protective of public health and safety for the six-cell disposal facility under consideration. For consistency, the methods of analyses and the exposure scenario used to predict the performance of a six-cell disposal facility were identical to those used in the Remedjal Investigation and Feasibility Study (RL/FS) and its Addendum (DOE 1998a, DOE 1998b) to develop the approved WAC. To take advantage of new information and design changes departing from the conceptual design, the modeling domain and model calibration were updated from those used in the RL/FS and its Addendum. It should be noted that this analysis is not intended to justify or propose a change in the approved WAC.

\section{EXPOSURE SCENARIO}

The exposure scenario used in the assessment consists of a resident farmer located near the confluence of Bear Creek and Northen Tributary-5 (NT-5). Bear Cretk surface water is used for watering livestock and irrigating crops, and drinking water is obtained from a well located near NT-5, between the EMWMF and Bear Creek. This exposure scenario is identical to the exposure scenario used to develop the approved WAC. Figure 1 shows the relative locations of the well, six-cell EMWMF, and Bear Creek and its tributaries used in the RI/FS and Addendum analyses.

\section{REVISION OF THE MODEL DOMAIN}

The RL/FS performance modeling domain and the larger modeling domain used in this analysis are also shown in Figure 1. Because the EMWMF is being constructed further to the north and east of the conceptual location of the EMWMF, the modeling domain was revised to include the full extent of the EMWMF in its present Iocation. Detailed descriptions of the groundwater modeling domain and associated specific features are given in Appendix B of the Engineering Feasibility Plan for Groundwater Suppression at the Environmental Management Waste Management Facility, Oak Ridge, Tennessee [Bechtel Jacobs Company LLC (BJC) 2003] and 
the subsequent report [Jacobs Engineering Group, Inc. (JEG) 2010]. Essential features of the revised modeling domain are:

- The refined model grid is extended north to the top of Pine Ridge (a zero flow groundwater modeling boundary);

- The western groundwater hydraulic head boundary is moved from its previous location in proximity to NT- 5 to the west, past NT- 6 .

Additional changes in the modeling that could influence the projected performance of the EMWMF include:

- A change in the cover design that reduced the rate of infiltration through the waste (DOE 2001b);

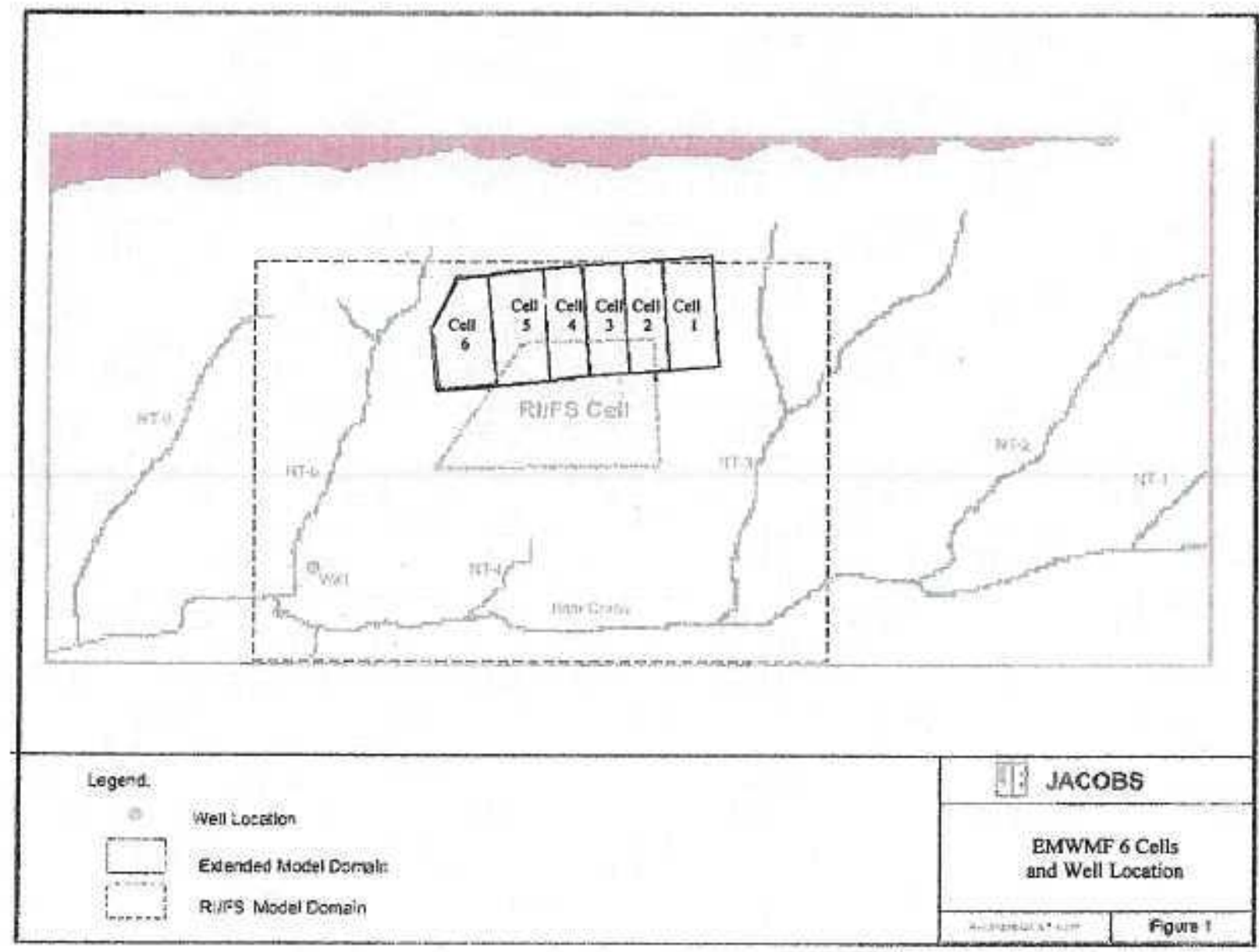

Figure 1. Original modeling domain and the modeling domain used in this analysis, and locations of RI/FS and Addendum disposal facility, EMWMF Cells 1 through 6, and receptor well. 
- The addition of an underdrain beneath Cell 3 that was installed in late 2003 and early 2004;

- An increase in the total plan area of the EMWMF;

- All the engineering and design features that are in place and proposed: and

- Calibration of the groundwater model to newly acquired groundwater and geological data up to date.

\section{MODELING CONTAMINANT MIGRATION}

The following analyses were performed under the assumption that Cells 1 through 6 have been closed and capped:

- The groundwater flow fields of the revised modeling domain, adjusted for the proposed build-out of the EMWMF to sis cells, were assessed using the MODFLOW groundwater model (McDonald and Harbaugh 1988, Jacobs 2010).

- Groundwater flow paths and particle travel times from cells to surface discharge locations were determined using the MODPATH model (Pollock 1989).

- Solute transport calculations were made for movement of contaminants from the disposal facility to the residential well using MT3D (Zheng 1990).

- Dilution factors (DFs) for Bear Creek and the residential well were calculated using the revised modeling domain for the EMWMF. .The DFs are defined as the ratios of the peak steady-state contaminant concentrations in the Bear Creek water or water continuously pumped (250 gallons per day) from the well to unit contaminant concentrations entering the groundwater beneath the disposal facility.

\section{METHOD OF RISK AND DOSE ASSESSMENT}

The projected peak risks and doses from radioactive or hazardous constituents in the first 100,000 years after closure were calculated for consumption of drinking water from a well and using Bear Creek surface water for agricultural purposes with a concentration of contaminants in the waste (source term) of $1 \mathrm{Ci} / \mathrm{m}^{3}$ (curie per cubic meter) for radioactive constituents and 1 $\mathrm{kg} / \mathrm{m}^{3}$ (kilogram per cubic meter) for toxicological constituents, respectively. Those risks and doses were scaled according to the approved WAC concentrations to determine the risks and doses that could occur if each constituent was present alone in the EMWMF at the WAC concentration. Those risks and doses were then compared to the risk and dose criteria upon which the WAC are based. 
The calculations were performed in the following steps:

1. PATHRAE [Rogers and Associates Engineering (RAE) 1995a and 1995b] calculations were performed to determine the equivalent annual water consumption per year for Bear Creck [defined as the Equivalent Uptake (EU)]. This equivalent water consumption corresponds to scaling the use of Bear Creek water for drinking and agricultural purposes to an equivalent annual drinking water ingestion that would give the same annual constituent uptake as calculated to come from all pathways. Since drinking water in the resident farmer exposure scenario will be supplied by a well rather than Bear Creek, the annual drinking water volume of $730 \mathrm{l} / \mathrm{yr}$ to be supplied by the well is subtracted from the Bear Creek EU to estimate the effective drinking water ingestion that can be associated with agricultural uses for Bear Creek surface water. The PATHRAE calculations also provide peak concentrations of contaminants in Bear Creek water corresponding to a unit source term, the corresponding peak doses or risks associated with those concentrations, and the times of occurrence of the peak concentrations.

2. The calculated DFs for Bear Creek and the residential well were used for scaling the constituent concentrations in Bear Creek to corresponding well concentrations. Using the 6cell EMWMF configuration and design, the ratio of the constituent concentrations in the well to those in Bear Creek is $\mathrm{DF}$ Well$_{/} / \mathrm{DF}_{\text {Cnek }}=0.00060 / 0.0045=0.13$.

3. The peak effective risk or dose was calculaled as the risk or dose due to ingestion of $730 \mathrm{l} / \mathrm{yr}$ per year of water drawn from the well, plus the consumption of agricultural products and livestock irrigated or watered with Bear Creek surface water. The latter is calculated by subtracting $730 \mathrm{~V} / \mathrm{yr}$ of water from the EU for Bear Creek water that is calculated by the PATHRAE computer code. Thus:

Peak Effective Risk or Dose = Peak Bear Creck Risk or Dase $\mathrm{x}$

$$
\left[\mathrm{EU}-730+\left(\mathrm{DF}_{W_{\mathrm{e}} / \mathrm{DF}} / \mathrm{F}_{\mathrm{Crek}}\right) \times 730\right] / \mathrm{EU}
$$

where $D_{\text {well }}$ and $\mathrm{DF}_{\text {Creck }}$ are the dilution factors calculated for the well and the creek. respectively, and the peak Bear Creek risk or dose correspond to ingestion of Bear Creek water at the annutal $\mathrm{EU}$ rate.

Radioactive Constituents - Risk

The Peak Bear Creek Risk for radioactive constituents is,

Peak Bear Creek Risk $=$ Peak Bear Creek concentration $x$ EU $x$

Slope Facior $\mathrm{x} 30$ years exposure duration. 
whcre the peak Bear Creek risk is calculated directly by the PATHRAE-RAD computer code using slope factors ['ncremental Lifetime Cancer Risk (ILCR) /pCi] obtained from the EPA's Health Effects Assessment Summary Tables.

Hazardous Constituents - Risk and Dose

Peak Bear Creek Risk $=$ Peak Bear Creck Lifetime Intake of Carcinogens $x$

Slope Factor.

Peak Bear Creek Lifetime Intake for Carcinogens =

Peak Bear Creek Concentration $x$

EU $\times 30$ years exposure duration /

[70 kg body weight $x 365$ days per year $x 70$ year life]

and,

Peak Bear Creek Daily Intake (Dose) for Non-Carcinogens =

Peak Bear Creek Concentration x EU /

[70 $\mathrm{kg}$ body weight $x 365$ days per year],

where the peak Bear Creek daily intake for carcinogens is calculated using PATHRAE-HAZ generated data and the formula immediately above.

The peak risks and doses resulting from constituent concentrations in the waste equal to the WAC concentrations were then calculated as follows:

For radioactive constituents:

Peak Effective Risk for constituents in the waste at the WAC level =

[Peak Effective Risk from a $1 \mathrm{Ci} / \mathrm{m}^{3}$ source $\mathrm{x}$ WAC] $/ 6.25 \times 10^{5}$

where the WAC are expressed in picuries per gram (pCi/g) and the factor $6.25 \times 10^{5}$ results from unit conversions.

For hazardous constituents:

Peak Effective Risk or Dose for constituents in the waste at the WAC level = 


\section{[Peak Effective Risk or Dose from a $1 \mathrm{~kg} / \mathrm{m}^{3}$ source x WACl $/ 625$}

where the WAC are expressed in milligrams per kilogram ( $\mathrm{mg} / \mathrm{kg}$ ) and the factor of 625 comes from unit conversions.

\section{MODELING RESULTS FOR THE EMWMF CELLS I THROUGH 6 WITH UNDERDRAIN}

Peak risk and dose calculations for the EMWMF Cells 1 through 6 using the PATHRAE-RAD and PATHRAE-HAZ environinental pathway analysis computer codes are based on the following input parameters and data, some of which were generated using MODFLOW and MT3D for the extended modeling domain.

Volume-weighted average waste height $50.6 \mathrm{ft}=15.4 \mathrm{~m}$

Effective Cells $1-6$ horizontal dimensions $=2,584 \mathrm{ft} \times 451 \mathrm{ft}=788 \mathrm{~m} \times 137 \mathrm{~m}$

Waste footprint area $=1,190,401 \mathrm{ft}^{2}=1 \mathrm{t} 0,592 \mathrm{~m}^{2}$

Waste volume $=5.90 \times 10^{7} \mathrm{ft}^{3}=2.18 \times 10^{6} \mathrm{cy}=1.67 \times 10^{6} \mathrm{~m}^{3}$

Infiltration rate $=0.36$ inches $/$ year $=0.91 \mathrm{~cm} /$ year

Volume-weighted average distance to Bear Creck or to a drainage feature that gives quick access to Bear Creek $=331 \mathrm{ft}=101 \mathrm{~m}$

Creek dilution factor $\left(\mathrm{DF}_{(\mathrm{Crex})}\right)=4.5 \times 10^{-3}$

Well dilution factor $\left(\mathrm{DF}_{\left.\mathrm{Wel}_{\mathrm{e}}\right)}\right)=6.0 \times 10^{-4}$

The peak risks and doses calculated using the PATHRAE code for Cells 1 through 6 , based on unit source terms, ane given in Tables 1 and 2 for the radioactive and hazardous constituents, respectively. The PATHRAE input parameters used in these calculations and summaries of output results are given in Attachment 1. Note that the peak doses in Bear Creek in Table 2, as given by PATHRAE, are always higher than their corresponding peak effective doses because the former are calculated based on the assumption that Beak Creek water is used for all purposes. The constituent concentrations in the well are lower than in Bear Creek and, since most of the effective uplake for most constituents is from drinking water (i.e., water from the well), the effective dose is usually considerably lower.

The projected peak risks and doses from the proposed 6-cell EMWMF, using the approved WAC as the source terms, are given in Tables 3 and 4 . Table 3 and 4 also list the time to the projected concentration peak as well as the risk and dose criterion for each contaminant. These are the same criteria as were used to develop the approved WAC. 


\section{CONCLUSION}

If the approved list of WAC constituents that have finite WAC values (given in Revised Table A.1: Analytic WAC Linits, http://bechteljacobs.org/webindex.html\#3) are assumed to be separately in the EMWMF at the maxinum allowable concentrations prescribed by their respective WAC, the resultant risks and doses to the receptor are summarized in Tables 3 and 4. It can be seen that those risks and doses will not exceed the current WAC criteria for any of the constituents.

The aralyses that led to the development of the projected WAC in Table A.l showed that, for the original 4-cell configuration of the EMWMF, the concentrations of constituents in the creek water and the well water would be approximately the same. Those analyses also demonstrated that, for the vast majority of the constituents, most of the risks and doses to the receptor comes from drinking well water. Any major reduction in constituent concentrations in the groundwater at the well will greatly reduce projected risks and doses. Groundwater modeling shows that the addition of an underdrain beneath Cell 3 that diverts much of the leachate directly to Bear Creek via the remnant of NT-4, as well as the extension of the EMWMF westward, significantly reduces constituent concentrations in the well water.

The addition of Cells 5 and 6 , with the presence of an underdrain under Cell 3, will result in lower risks and doses than were expected when the WAC in Table A.I were developed. Therefore, the existing WAC are conservatively low for the proposed 6-cell configuration. 
Table 1. Peak Effective Risks for the EMWMF for Radioactive Constituents (rlsks based on a $1 \mathrm{C} / \mathrm{m}^{3}$ concentration in the waste)

\begin{tabular}{|c|c|c|c|c|c|}
\hline Nuclide & $\begin{array}{c}\text { Peak } \\
\text { Concentration in } \\
\text { Bear Creek } \\
(\mathrm{pCl} / \mathrm{L})\end{array}$ & $\begin{array}{l}\text { Ingestion } \\
\text { Slope Factor } \\
\left(1 / \mathrm{pC}_{1}\right)\end{array}$ & $\begin{array}{l}\text { Equivalent } \\
\text { Uptake } \\
\text { (L/yr) }\end{array}$ & $\begin{array}{cc}\text { Peak } \\
\vdots \\
\text { Effective } \\
\text { Risk } \\
\text { (ILCR) }\end{array}$ & $\begin{array}{c}\text { Time of } \\
\text { Projected } \\
\text { Peak } \\
(y r)\end{array}$ \\
\hline $\mathrm{H}-3$ & $4.24 \mathrm{E}-02$ & $715 \mathrm{E}-14$ & $1.17 \mathrm{E}+03$ & $4.85 \mathrm{E}-11$ & 330 \\
\hline $\mathrm{C}-14$ & $2.12 \mathrm{E}+06$ & $103 \mathrm{E}-12$ & $956 \mathrm{E}+02$ & $2.12 \mathrm{E}-02$ & 380 \\
\hline Tc-99 & $1.9 \mid \mathrm{E}+06$ & $1.40 \mathrm{E}-12$ & $7.40 \mathrm{E}+02$ & $8.59 \mathrm{E}-03$ & 410 \\
\hline I-129 & $984 \mathrm{E}+05$ & $1.84 \mathrm{E}-10$ & $8.38 \mathrm{E}+02$ & $1.1 / \mathrm{E}+00$ & 900 \\
\hline $\mathrm{U}-233$ & $5.66 \mathrm{E}+04$ & $4.48 \mathrm{E}-11$ & $38 \mathrm{E}+02$ & $799 \mathrm{E}-03$ & 45,000 \\
\hline $\mathrm{U}-234$ & $606 \mathrm{E}+04$ & 4.44E-11 & $738 E+02$ & $8.48 E-03$ & 45,000 \\
\hline $\mathrm{U}-235$ & $6.89 \mathrm{E}+04$ & $470 \mathrm{E}-1 \mathrm{I}$ & $7.38 \mathrm{BE}+02$ & $1.02 \mathrm{E}-02$ & 53,000 \\
\hline$U-236$ & $6.88 \mathrm{E}+04$ & $4.21 \mathrm{~L}-11$ & $7.38 E+02$ & $9.13 E-0,3$ & 46,000 \\
\hline $\mathrm{U}-238$ & $6.89 \mathrm{E}+04$ & $620 \mathrm{E}-11]$ & $7.38 \mathrm{E}+02$ & $1.35 \mathrm{E}-02$ & 53,000 \\
\hline $\mathrm{Np}-2,37$ & $4.80 E+04$ & $2,95 \mathrm{E} / 10$ & $734 \mathrm{E}+02$ & $4.29 \mathrm{E}-02$ & 100,000 \\
\hline $\mathrm{Pu}-239$ & $299 \mathrm{E}+03$ & $3, J 6 \mathrm{E}-10$ & $733 \mathrm{E}+02$ & $284 \mathrm{E}-03$ & 95,000 \\
\hline $\mathrm{Pu}-240$ & $2.19 \mathrm{E}+00$ & $3.15 \mathrm{E}-10$ & $733 \mathrm{E}+02$ & $2,07 \mathrm{E}-06$ & 92,000 \\
\hline$A m-241$ & $\mathbf{a}$ & $3.28 \mathrm{E}-10$ & سيد & س. & a \\
\hline
\end{tabular}

Note The ratio of the dilution factors $D F_{\text {uell }}$ to the $\mathrm{DF}_{\text {trefk }}$ is 013

$a=$ The PATHRAE code did not indicate a non-zero value for the concentration of Am-241 at any time before 100,000 years. The $A m-241$ nuclide has the same $K_{d ;}$ as the Pu-241 nuclide so it should arrive at key locations at the same time. However, since Am241 has a half - life of 432 years, less than one tenth of that for Pu-240, much more of it will decay away before about 84,000 years, the time at which Pu- 240 begins to show up in a discernable concentration. Therefore it is concluded that the WAC for Am-24l should be set at the specific activity of that nuclide. 
Table 2. Peak Effective Risks and Doses for the EMWMF for Hazardous Constiluents (based on a $1 \mathrm{~kg} / \mathrm{m}^{3}$ concentration in the waste)

\begin{tabular}{|c|c|c|c|c|c|c|c|}
\hline Constituent & $\begin{array}{c}\text { Peak } \\
\text { Dose } \\
\text { in Bear } \\
\text { Creek } \\
\text { (mg/kg- } \\
\text { day) } \\
\end{array}$ & $\begin{array}{c}\text { Peak } \\
\text { Concentration } \\
\text { in Bear Creek } \\
(\mathrm{mg} / \mathrm{L}) \\
\end{array}$ & $\begin{array}{c}\text { Reference } \\
\text { Dese } \\
\text { (mg/kg-day) }\end{array}$ & $\begin{array}{c}\text { Equivalent } \\
\text { Uptake } \\
\text { (L/yr) }\end{array}$ & $\begin{array}{c}\text { Slope } \\
\text { Factor } \\
(1 / \mathrm{mg} / \mathrm{kg}-\mathrm{d})\end{array}$ & $\begin{array}{c}\text { Peak } \\
\text { Effective } \\
\text { Risk } \\
\text { (JLCR) }\end{array}$ & $\begin{array}{c}\text { Peak } \\
\text { Effective } \\
\text { Dase } \\
\text { (mg/kg- } \\
\text { day) }\end{array}$ \\
\hline Acenaphthene & * & $*$ & * & $*$ & * & * & $*$ \\
\hline Acenaphythylene & $2.04 \mathrm{E}-03$ & $7.09 \mathrm{E}-02$ & $6.00 \mathrm{E}-02$ & $7.34 \mathbf{E}+02$ & & & $2.75 \mathrm{E}-04$ \\
\hline Acetone & $3.96 \mathrm{E}-01$ & $1.38 \mathrm{E}+01$ & $1.00 \mathrm{E}-01$ & $7 \overline{33 \mathrm{E}+02}$ & & & $5.92 \mathrm{E}-02$ \\
\hline Aldrin & $*$ & 木 & $*$ & $*$ & * & * & $*$ \\
\hline Antimony & $4.14 \mathrm{E}-03$ & $1.44 \mathrm{E}-0]$ & $4.00 \mathrm{E}-04$ & $7.33 \mathrm{E}+02$ & & & $5.53 \mathrm{E}-04$ \\
\hline Aruclor 1221 & * & $*$ & $*$ & $*$ & * & * & $*$ \\
\hline Aroclor 1232 & $6.10 \mathrm{E}-04$ & $2.1 \mathrm{E}-02$ & * & $7.33 \mathrm{E}+02$ & $4.00 \mathrm{E}+01$ & $1.38 \mathrm{E}-05$ & \\
\hline Bariın & $*$ & $*$ & * & $*$ & $*$ & $*$ & \# \\
\hline Boron & $2.03 \mathrm{E}-02$ & $6.9 \overline{5 \mathrm{E}-04}$ & $2.00 \mathrm{E}-01$ & $7.48 \mathrm{E}+02$ & & & $3.14 \mathrm{E}+03$ \\
\hline Alpha-BHC & $1.01 \mathrm{E}-03$ & $3.52 \mathrm{E}-02$ & & $7.34 \mathrm{E}+02$ & $6.30 \mathrm{E}+00$ & $3.86 \mathrm{E}-04$ & \\
\hline Beta-BHC & $1.01 \mathrm{E}-03$ & $3.52 \mathrm{E}-02$ & & $7.33 \mathrm{E}+02$ & $1.80 \mathrm{E}+00$ & $1.06 \mathrm{E}-04$ & \\
\hline Delta-BHC & $1.01 \mathrm{E}-03$ & $3 . \overline{2 \mathrm{E}-02}$ & & $7.35 \mathrm{E}+02$ & $1.80 \mathrm{E}+00$ & $1.04 \mathrm{E}-04$ & \\
\hline Benzene & $4.27 \mathrm{E}-02$ & $1.49 \mathrm{E}+00$ & & $7,33 \mathrm{E}+02$ & $2.90 \mathrm{E}-02$ & $1.85 \mathrm{E}-01$ & \\
\hline Benzoic Acid & $4.30 \mathrm{E}-01$ & $1.50 \mathrm{E}+0 \mathrm{l}$ & $4.00 \mathrm{E}+00$ & $7.33 \mathrm{E}+02$ & & & $5.65 \overline{\mathrm{E}}-02$ \\
\hline Benzyl Alcohol & $4.23 \mathrm{E}-01$ & $1.47 \mathrm{E}+01$ & $3.00 \mathrm{E}-01$ & $7.33 \mathrm{E}+02$ & & & $5.74 \mathrm{E}-02$ \\
\hline Carbazole & $2.28 \bar{E}-04$ & $7.93 \mathrm{E}-03$ & & $7.34 \mathrm{E}+02$ & $2.00 \mathrm{E}-02$ & $4.04 \mathrm{E}-02$ & \\
\hline Carbon Tetrachloride & $3.37 \mathrm{E}-02$ & $1.17 \mathrm{E}+00$ & $7.00 \mathrm{E}-04$ & $7.33 \mathrm{E}+02$ & $1.30 \mathrm{E}-01$ & $3.06 \mathrm{E}-02$ & $3.78 \mathrm{E}-04$ \\
\hline Chlorobenzene & $6.29 \mathrm{E}-02$ & $2.19 \mathrm{E}+00$ & $2.00 \mathrm{E}-02$ & $7.33 \mathrm{E}+02$ & & & $8.42 \mathrm{E}-03$ \\
\hline Chlordane & $*$ & $*$ & $*$ & $*$ & * & * & $*$ \\
\hline Chlotoform & $1.02 \mathrm{E}-01$ & $3.56 \mathrm{E}+0$ & $1.00 \mathrm{E}-02$ & $7.33 \mathrm{E}+02$ & $6.10 \mathrm{E}-03$ & $3.06 \mathrm{E}-2$ & $3.57 \mathrm{E}-05$ \\
\hline Chromium III & $8.30 \mathrm{E}-03$ & $2.72 \mathrm{E}-01$ & $1.00 \mathrm{E}+00$ & $7.79 \mathrm{E}+02$ & & & $1.53 \mathrm{E}-03$ \\
\hline m-Cresol & $3.15 \mathrm{E}-01$ & $1.10 \mathrm{E}-0 \mathrm{l}$ & $5.00 \mathrm{E}-02$ & $7.33 \mathrm{E}+02$ & & & $4.21 \mathrm{E}-02$ \\
\hline o-Cresol & $2.35 \mathrm{E}-01$ & $8.18 \mathrm{E}+00$ & $5.00 \mathrm{E}-02$ & $7.33 \mathrm{E}+02$ & & & $3.14 \mathrm{E}-02$ \\
\hline
\end{tabular}


Table 2. Peak Effective Risks and Doses for the EMWMF for Hazardous Constituents (based on a $1 \mathrm{~kg} / \mathrm{m}^{3}$ concentration in the waste)

\begin{tabular}{|c|c|c|c|c|c|c|c|}
\hline Constituent & $\begin{array}{c}\text { Peak } \\
\text { Dose } \\
\text { in Bear } \\
\text { Creek } \\
\text { (mg/kg- } \\
\text { day) }\end{array}$ & $\begin{array}{c}\text { Peak } \\
\text { Concentration } \\
\text { in Bear Creek } \\
(\mathrm{mg} / \mathrm{L})\end{array}$ & $\begin{array}{c}\text { Reference } \\
\text { Dose } \\
\text { (mg/kg-day) }\end{array}$ & $\begin{array}{c}\text { Equivalent } \\
\text { Uptake } \\
\text { (L/yr) }\end{array}$ & $\begin{array}{c}\text { Slope } \\
\text { Factor } \\
(1 / \mathrm{mg} / \mathrm{kg}-\mathrm{d})\end{array}$ & $\begin{array}{c}\text { Peak } \\
\text { Effective } \\
\text { Risk } \\
\text { (ILCR) }\end{array}$ & $\begin{array}{c}\text { Peak } \\
\text { Effective } \\
\text { Dose } \\
\text { (mg/kg- } \\
\text { day) }\end{array}$ \\
\hline p-Cresol & $3.19 \mathrm{E}-01$ & $1,11 \mathrm{E}+01$ & $5,00 \mathrm{E}-02$ & $7.33 \mathrm{E}+02$ & & & $4.26 \mathrm{E}-02$ \\
\hline Cyanide & $7.89 \mathrm{E}-03$ & $2.75 \mathrm{E}-0 \mathrm{I}$ & $2.00 \mathrm{E}-02$ & $7.33 \mathrm{E}+02$ & & & $1.05 \mathrm{E}-03$ \\
\hline Dibenz $[a, h]$ antracene & $*$ & $*$ & $*$ & $*$ & 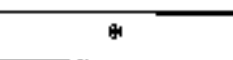 & * & $*$ \\
\hline 1-2-Dichlorobenzene & $1.01 \mathrm{E}-02$ & $3.52 \mathrm{E}-01$ & $9,00 \mathrm{E}-02$ & $7.33 E+02$ & & & $1.35 \mathrm{E}-03$ \\
\hline 1-3-Dichlorobenzene & $4.90 \mathrm{E}-03$ & $1.71 \mathrm{E}-0 \mathrm{l}$ & $8.90 \mathrm{E}-02$ & $7.34 \mathrm{E}+02$ & & & $6.60 \mathrm{E}-04$ \\
\hline 1-4-Dichlorobenzene & $1.03 \mathrm{E}-02$ & $3.58 \mathrm{E}-0 \mathrm{I}$ & $2.30 \mathrm{E}-01$ & $7.33 \mathrm{E}+02$ & $2.40 \mathrm{E}-02$ & $1.41 \mathrm{E}-05$ & $1.38 \mathrm{E}-03$ \\
\hline Diethyphthalate & $1.36 \mathrm{E}-01$ & $4.76 \mathrm{E}+00$ & 8.00E-01 & $7.33 \mathrm{E}+02$ & & & $1.82 \mathrm{E}-02$ \\
\hline Dimethylphthalate & $3.44 \mathrm{E}-01$ & $1.20 \mathrm{E}+01$ & $1.00 \mathrm{E}+01$ & $7.33 \mathrm{E}-+02$ & & & $4.59 \mathrm{E}-02$ \\
\hline Di-n-butylphthalate & $5.58 \mathrm{E}-01$ & $1.77 \mathrm{E}+01$ & $1.00 \mathrm{E}-01$ & $8.06 \mathrm{E}+02$ & & & $2.87 \mathrm{E}-02$ \\
\hline 2.4-Dinitrotoluene & $3.41 \mathrm{E}-02$ & $1.19 \mathrm{E}+00$ & $2.00 \mathrm{E}-03$ & $7.33 \mathrm{E}+02$ & $6.8 \bar{E}-04$ & $1.37 \mathrm{E}-03$ & $4.55 \mathrm{E}-03$ \\
\hline 2,6-Dinitrotoluene & $4.45 \mathrm{E}-02$ & $1.55 E+00$ & $1.00 \mathrm{E}-03$ & $7.33 \mathrm{E}+02$ & $6.80 \mathrm{E}-01$ & $1.73 \mathrm{E}-03$ & $5.94 \mathbf{E}-03$ \\
\hline Dieldrín & $2.63 \mathrm{E}-03$ & $8.10 \mathrm{E}-02$ & $5,00 \mathrm{E}-05$ & $8.29 \mathrm{E}+02$ & $1.30 \mathrm{E}+01$ & & $6.15 \mathrm{E}-04$ \\
\hline 2,4-D & $8.62 E-02$ & $3.00 \mathrm{E}+0$ & $1.00 \mathrm{E}-02$ & $7.33 \mathrm{E}+02$ & & & $1.15 \mathrm{E}-02$ \\
\hline DDD & $*$ & $*$ & $*$ & k & $*$ & & \\
\hline DDE & $5.70 \mathrm{E}-05$ & $1 . \overline{76 \mathrm{E}-02}$ & & $8.27 \mathrm{E}+02$ & $3.40 \mathrm{E}-0 \mathrm{I}$ & $1.93 \mathrm{E}-07$ & \\
\hline Endosulfan I & $5.69 \mathrm{E}-05$ & $1.98 \mathrm{E}-03$ & $6.00 \mathrm{E}-03$ & $7.34 \mathrm{E}+02$ & & & $7.67 \mathrm{E}-06$ \\
\hline Endosulfan II & 5.69E-05 & $1.98 \mathrm{E}-03$ & $6.00 \mathrm{E}-03$ & $7.34 \mathrm{E}+02$ & & & $7.67 \mathrm{E}-06$ \\
\hline Endrin & $3.19 \mathrm{E}-05$ & 1. L0E-03 & $3.00 \mathrm{E}-04$ & $7.40 \mathrm{E}+02$ & & & $4.52 \mathrm{E}-06$ \\
\hline Endrin Aldehyde & $3.19 \mathrm{E}-03$ & 1.10E-03 & $3,00 \mathrm{E}-04$ & $7.40 \mathrm{E}+02$ & & & $4.52 \mathrm{E}-06$ \\
\hline Endrin Ketone & $3.19 \mathrm{E}-03$ & ].10E-03 & $3.00 \mathrm{E}-04$ & $7.40 \mathrm{E}+02$ & & & $4.52 \mathrm{E}-06$ \\
\hline Hexachlorobenzene & $*$ & $*$ & $*$ & $*$ & $*$ & $*$ & $*$ \\
\hline Hexachloroethane & $6.33 \mathrm{E}-03$ & $2.20 \mathrm{E}-01$ & $1.00 \mathrm{E}-03$ & $7.34 \mathrm{E}+02$ & $1.40 \mathrm{E}-02$ & $5.14 \mathrm{E}-06$ & $8.53 \mathrm{E}-04$ \\
\hline Heplachlor & $4.10 \mathrm{E}-21$ & $1.40 \mathrm{E}-19$ & $5.00 \mathrm{E}-04$ & $7.37 \mathrm{E}+02$ & $4.50 \mathrm{E}+00$ & $6.16 \mathrm{E}-06$ & $3.17 \mathrm{E}-06$ \\
\hline
\end{tabular}


Table 2. Peak Effective Risks and Doses for the EMWMF for Hazardous Constituents (based on a $1 \mathrm{~kg} / \mathrm{m}^{3}$ concentration in the waste)

\begin{tabular}{|c|c|c|c|c|c|c|c|}
\hline Constituent & $\begin{array}{c}\text { Peak } \\
\text { Dose } \\
\text { in Bear } \\
\text { Creek } \\
\text { (mg/kg- } \\
\text { day) }\end{array}$ & $\begin{array}{c}\text { Peak } \\
\text { Concentration } \\
\text { in Bear Creek } \\
(\mathrm{mg} / \mathrm{L})\end{array}$ & $\begin{array}{c}\text { Reference } \\
\text { Dose } \\
\text { (mg/kg-day) }\end{array}$ & $\begin{array}{l}\text { Equivalent } \\
\text { Uptake } \\
\text { (L/yr) }\end{array}$ & $\begin{array}{c}\text { Slope } \\
\text { Factor } \\
(1 / \mathrm{mg} / \mathrm{kg}-\mathrm{d})\end{array}$ & $\begin{array}{c}\text { Peak } \\
\text { Effective } \\
\text { Risk } \\
\text { (ILCR) }\end{array}$ & $\begin{array}{c}\text { Peak } \\
\text { Effective } \\
\text { Dose } \\
\text { (mg/kg- } \\
\text { day) }\end{array}$ \\
\hline Hepiachlor Epoxide & $2.68 \mathrm{E}-05$ & $8.81 \mathrm{E}-04$ & $1.30 \mathrm{E}-05$ & $7.79 \mathrm{E}+02$ & $9.10 \mathrm{E}+00$ & $2.07 \mathrm{E}-05$ & $4.97 \mathrm{E}-06$ \\
\hline Isophorone & $4.27 \mathrm{E}-02$ & $1 . \overline{49 \mathrm{E}+00}$ & $2+00 \mathrm{E}-[0]$ & $7.33 \mathrm{E}+02$ & $9.50 \mathrm{E}-04$ & $2.40 \mathrm{E}-06$ & $5.88 \mathrm{E}-03$ \\
\hline Lead & $*$ & $\neq$ & $*$ & $*$ & $*$ & $*$ & * \\
\hline Lindane & $1.00 \mathrm{E}-03$ & $3.50 \mathrm{E}-02$ & $3.00 \mathrm{E}-04$ & $7 . \overline{35 E+02}$ & $1.30 \mathrm{E}+00$ & $7.68 \mathrm{E}-05$ & $1.7 \mathbf{E}-04$ \\
\hline Manganese & $*$ & $*$ & $*$ & $*$ & $*$ & $*$ & $*$ \\
\hline Molybdenum & $4.03 \mathrm{E}-03$ & $1.37 \mathrm{E}-01$ & $5.00 \mathrm{E}-03$ & $7.50 \mathrm{E}+02$ & & & $5.48 \mathrm{E}-04$ \\
\hline Methylene Chloride & $6.01 \overline{\mathrm{E}}-0 \mathrm{I}$ & $2.10 \mathrm{E}-01$ & $6.00 \mathrm{E}-02$ & $7.33 \mathrm{E}+02$ & $7.50 \mathrm{E}-03$ & $2.24 \mathrm{E}-05$ & $8.03 \overline{\mathrm{E}-02}$ \\
\hline Methylcyclohexane & $1.77 \mathrm{E}-03$ & $6.17 \mathrm{E}-02$ & $6.00 \mathrm{E}-02$ & $7.33 \mathrm{E}+02$ & & & $2.36 \mathrm{E}-04$ \\
\hline 2-Methylnapthalene & $3.11 \mathrm{E}-03$ & $1.08 \mathrm{E}-01$ & $4.00 \mathrm{E}-03$ & $7.33 \mathrm{E}+02$ & & & 4.19E-04 \\
\hline Nitrobenzene & $2.64 \mathrm{E}-01$ & $9.21 \mathrm{E}+00$ & $5.00 \mathrm{E}-04$ & $7.33 \mathrm{E}+02$ & & & $3.54 \mathrm{E}-02$ \\
\hline 4-Nitrobenzamine & $1.35 \overline{\mathrm{E}-09}$ & $4.71 \mathrm{E}-08$ & $3.00 \mathrm{E}-03$ & $7.33 \mathrm{E}+02$ & $2.10 \mathrm{E}-02$ & $1,62 \mathrm{E}-12$ & $1.80 \mathrm{E}-10$ \\
\hline $\begin{array}{l}\text { N-nitroso-di-n- } \\
\text { propolyamine }\end{array}$ & $1.74 \mathrm{E}-01$ & $6.05 \mathrm{E}+00$ & & $7.33 \mathrm{E}+02$ & $7.00 \mathrm{E}+00$ & $7.18 \mathrm{E}-02$ & \\
\hline N-Nitrosodiphenylamine & $4.42 \mathrm{E}-03$ & $1.54 \mathrm{E}-01$ & $2.00 \mathrm{E}-02$ & $7.33 \mathrm{E}+02$ & $4.90 \mathrm{E}-03$ & $1.24 \mathrm{E}-06$ & $5.90 \mathrm{E}-04$ \\
\hline Napthalcne & $3.92 \mathrm{E}-03$ & $1.37 \mathrm{E}-01$ & $3.60 \mathrm{E}-02$ & $7.33 \mathrm{E}+02$ & & & $5.24 \mathrm{E}-04$ \\
\hline 2-Nitrophenol & $9.16 \mathrm{E}-02$ & $3.19 \overline{\mathbf{E}}+00$ & $6.20 \mathrm{E}-02$ & $7,33 \mathrm{E}+02$ & & & $3.75 \mathrm{E}-04$ \\
\hline 4-Nitrophenol & $7.70 \mathrm{E}-02$. & $2.68 \mathrm{E}+00$ & $6.20 \mathrm{E}-02$ & $7.33 \mathrm{E}+02$ & & & $1.03 \mathrm{E}-02$ \\
\hline Phenol & $1.82 \mathrm{E}-01$ & $6.33 \mathbf{E}+00$ & $6.00 \mathrm{E}-01$ & $7.33 \mathbf{E}+02$ & & & $6.48 \mathrm{E}-02$ \\
\hline Pyridine & $4.66 \mathrm{E}-01$ & $1.63 \mathrm{E}+01$ & $1.00 \mathrm{E}-03$ & $7.33 \mathrm{E}+02$ & & & $6.22 \mathrm{E}-02$ \\
\hline Selenium & $9.16 \mathrm{E}-03$ & $2,03 \mathrm{E}-01$ & $5.00 \mathrm{E}-03$ & $1.31 \mathrm{E}+03$ & & & $4.02 \mathrm{E}-03$ \\
\hline Strontium & $6.29 \mathrm{E}-03$ & $1.78 \mathrm{E}-01$ & $6.00 \mathrm{E}-01$ & $7.94 E+02$ & & & L. $18 \mathrm{E}-03$ \\
\hline Tin & $3.17 \mathrm{E}-02$ & $2.03 \mathrm{E}-01$ & $6.00 \mathrm{E}-01$ & $7.91 \mathbf{E}+02$ & & & $5.99 \mathrm{E}-03$ \\
\hline Tetrachloroethene & $1.08 \mathrm{E}-02$ & $1.02 \mathrm{E}+00$ & $1.00 \mathrm{E}-02$ & $7.33 E+02$ & $5.20 \mathrm{E}-02$ & $3.31 \mathrm{E}-05$ & $1.48 \mathrm{E}-03$ \\
\hline 2,3,4,6-Tetrachlorophenol & $*$ & $*$ & $*$ & * & $*$ & $*$ & $*$ \\
\hline
\end{tabular}


Table 2. Peak Effective Risks and Doses for the EMWMF for Hazardous Constituents (based on a $1 \mathrm{~kg} / \mathrm{m}^{3}$ concentration in the waste)

\begin{tabular}{|c|c|c|c|c|c|c|c|}
\hline Constituent & $\begin{array}{c}\text { Peak } \\
\text { Dose } \\
\text { in Bear } \\
\text { Creek } \\
\text { (mg/kg- } \\
\text { day) }\end{array}$ & $\begin{array}{c}\text { Peak } \\
\text { Concentration } \\
\text { in Bear Creek } \\
(\mathrm{mg} / \mathrm{L})\end{array}$ & $\begin{array}{c}\text { Reference } \\
\text { Dose } \\
\text { (mg/kg-day) }\end{array}$ & $\begin{array}{c}\text { Equivalent } \\
\text { Uptake } \\
\text { (L/yr) }\end{array}$ & $\begin{array}{c}\text { Slope } \\
\text { Factor } \\
(1 / \mathrm{mg} / \mathrm{kg}-\mathrm{d})\end{array}$ & $\begin{array}{c}\text { Peak } \\
\text { Effective } \\
\text { Risk } \\
\text { (JLCR) }\end{array}$ & $\begin{array}{c}\text { Peak } \\
\text { Effective } \\
\text { Dose } \\
\text { (mg/kg- } \\
\text { day) }\end{array}$ \\
\hline 1,2.4-Trichlorbenzene & $7.21 \mathrm{E}-03$ & $2.51 \mathrm{lE}-01$ & $5.00 \mathrm{E}-02$ & $7.35 \mathrm{E}+02$ & & & $9.63 \mathrm{E}-03$ \\
\hline Trichloroethene & $2.88 \mathrm{E}-02$ & $1.00 \mathrm{E}+00$ & & $7.33 \mathrm{E}+02$ & $1,10 \mathrm{E}-02$ & $1.87 \mathrm{E}-05$ & \\
\hline 2,4,6-Trichlorophenol & $1,00 \mathrm{E}-01$ & $3.49 \mathrm{E}+00$ & $1.00 \mathrm{E}-02$ & $7.34 E+02$ & $1.1 \mathrm{E}-02$ & $6.37 \mathrm{E}-05$ & $1.35 \mathrm{E}-02$ \\
\hline Toluene & $1.29 \mathrm{E}-02$ & $4 . \overline{49 \mathrm{E}-01}$ & $2.00 \mathrm{E}-01$ & $7.33 \mathrm{E}+02$ & & & $3.58 \mathrm{E}-03$ \\
\hline 2,4,5-TP(Silvex) & $2.53 \mathrm{E}-02$ & $8.81 \mathrm{E}-01$ & $8,00 \mathrm{E}-03$ & $7.34 \mathrm{E}+02$ & & & $3.41 \mathrm{E}-03$ \\
\hline $\mathrm{U}-233^{\mathrm{a}}$ & $1.47 \mathrm{E}-03$ & $5.11 \mathrm{E}-02$ & $3.00 \mathrm{E}-\overline{03}$ & $7.37 \mathrm{E}+02$ & See Table 3 & & $2.03 \mathrm{E}-04$ \\
\hline $\mathrm{U}-234^{\mathrm{a}}$ & $1.63 \mathrm{E}-03$ & $5.69 \mathrm{E}-02$ & $3,00 \mathrm{E}-03$ & $7.37 \mathrm{E}+02$ & See Table 3 & & $2.25 \mathrm{E}-04$ \\
\hline $\mathrm{U}-235^{\mathrm{a}}$ & $1.99 \mathrm{E}-03$ & $6.89 \mathrm{E}-02$ & $3.00 \mathrm{E}-03$ & $7.37 \mathrm{E}+02$ & See Table 3 & & $2.75 \mathrm{E}-04$ \\
\hline $\mathrm{U}-236^{\mathrm{a}}$ & $1.98 \mathrm{E}-03$ & $6.87 \mathrm{E}-02$ & $3,00 \mathrm{E}-03$ & $7.37 \mathrm{E}+02$ & See Table 3 & & $2.74 \mathrm{E}-04$ \\
\hline $\mathrm{U}-238^{\mathrm{a}}$ & $1.99 \mathrm{E}-03$ & $6.89 \mathrm{E}-02$ & $3.00 \mathrm{E}-03$ & $7.37 \mathrm{E}+02$ & See Table 3 & & $2.75 \mathrm{E}-04$ \\
\hline Vanadium & $*$ & $*$ & $*$ & $*$ & $*$ & $*$ & $*$ \\
\hline Vinyl Chloride & $1.50 \mathrm{E}-01$ & $5.24 \mathrm{E}+00$ & $3.00 \mathrm{E}-03$ & $7.33 \mathrm{E}+02$ & $1.40 \mathrm{E}+00$ & $1.21 \mathrm{E}-02$ & $2.00 \mathrm{E}-02$ \\
\hline Xylene(mixture) & $1.34 \mathrm{E}-02$ & 4.67E-01 & $2.00 \mathrm{E}-01$ & $7,33 \mathrm{E}+02$ & & & $1.79 \mathrm{E}-03$ \\
\hline Acetonitrile & $6.71 \mathbf{E}-01$ & $2.34 \mathrm{E}+01$ & $6.00 \mathrm{E}-03$ & $7.33 \mathrm{E}+02$ & & & $6.95 \mathrm{E}-02$ \\
\hline Acetophenone & $4.26 \mathrm{E}-01$ & $1.49 \mathrm{E}+01$ & $1,00 \mathrm{E}-01$ & $7.33 \mathrm{E}+02$ & & & $5.69 \mathrm{E}-02$ \\
\hline Acrolein & $6.66 \mathrm{E}-01$ & $2.32 \mathrm{E}+0 \mathrm{I}$ & $5,00 \mathrm{E}-04$ & $7.33 \mathrm{E}+02$ & & & $6.90 \mathrm{E}-02$ \\
\hline Acrylonitrile & $6.59 \mathrm{E}-01$ & $2,30 \mathrm{E}+01$ & $1.00 \mathrm{E}-03$ & $7,33 \mathrm{E}+02$ & $5.40 \mathrm{E}-01$ & $2.04 \mathrm{E}-02$ & $6.83 \mathrm{E}-02$ \\
\hline Butylbenzene & $7.75 \mathrm{E}-03$ & $2.70 \mathrm{E}-0 \mathrm{I}$ & $3.80 \mathrm{E}-02$ & $7.33 \mathrm{E}+02$ & & & $3.17 \mathrm{E}-05$ \\
\hline Ethylchloride & $5.20 \mathrm{E}-01$ & $1.81 \mathrm{E}+0 \mathrm{l}$ & $4.00 \mathrm{E}-01$ & $7.33 \mathrm{E}+02$ & $2.90 \mathrm{E}-03$ & $8.62 \mathrm{E}-\overline{05}$ & $6.95 \mathrm{E}-02$ \\
\hline 1-Hexanol & $4.35 \mathrm{E}-01$ & $1.52 \mathrm{E}+01$ & $4.00 \mathrm{E}-02$ & $7.33 \mathrm{E}+02$ & & & $5.81 \mathrm{E}-02$ \\
\hline 2-Hexanone & $4.3 \overline{\mathrm{E}-01}$ & $1.52 \mathrm{E}+0 \mathrm{I}$ & $4.00 \mathrm{E}-02$ & $7.33 \mathrm{E}+02$ & & & $5.81 \mathrm{E}-02$ \\
\hline $\begin{array}{l}\text { Trimethylbenzene } \\
\text { (mixcd isomers) }\end{array}$ & $5.82 \mathrm{E}-03$ & $2.03 \mathrm{E}-01$ & $5.00 \mathrm{E}-02$ & $7.34 \mathrm{E}+02$ & & & $1,02 \mathrm{E}-03$ \\
\hline Dibenzofuran & $1.70 \mathrm{E}-01$ & $5,94 \mathrm{E}+00$ & $4.00 \mathrm{E}-03$ & $7.33 \mathrm{E}+02$ & & & $2.93 \mathrm{E}-02$ \\
\hline
\end{tabular}


Table 2. Peak Effective Risks and Doses for the EMWMF for Hazardous Constituents (based on a $1 \mathrm{~kg} / \mathrm{m}^{3}$ concentration in the waste)

\begin{tabular}{|c|c|c|c|c|c|c|c|}
\hline Constituent & $\begin{array}{c}\text { Peak } \\
\text { Dose } \\
\text { in Bear } \\
\text { Creek } \\
(\mathrm{mg} / \mathrm{kg} \text { - } \\
\text { day) }\end{array}$ & $\begin{array}{c}\text { Peak } \\
\text { Concentration } \\
\text { in Bear Creek } \\
(\text { mg/L) }\end{array}$ & $\begin{array}{c}\text { Reference } \\
\text { Dose } \\
\text { (mg/kg-day) }\end{array}$ & $\begin{array}{c}\text { Equivalent } \\
\text { Uptake } \\
\text { (L/yr) }\end{array}$ & $\begin{array}{c}\text { Slope } \\
\text { Factor } \\
(1 / \mathrm{mg} / \mathrm{kg}-\mathrm{d})\end{array}$ & $\begin{array}{c}\text { Peak } \\
\text { Effective } \\
\text { Risk } \\
\text { (ILCR) }\end{array}$ & $\begin{array}{c}\text { Peak } \\
\text { Effective } \\
\text { Dose } \\
\text { (mg/kg- } \\
\text { day) }\end{array}$ \\
\hline 2,4-Dimethylphenol & $2.96 \mathrm{E}-02$ & $\mathrm{t} .03 \mathrm{E}+00$ & $2.00 \mathrm{E}-02$ & $7.33 \mathrm{E}+02$ & & & $3.95 \mathrm{E}-03$ \\
\hline Benzidine & $1.41 \mathrm{E}-02$ & $4.19 \mathrm{E}-01$ & $3.00 \mathrm{E}-03$ & $7,33 \mathrm{E}+02$ & $2.30 \mathrm{E}+02$ & $1.85 \mathrm{E}-01$ & $1.88 \mathrm{E}-03$ \\
\hline Methanol & $6.69 \overline{\mathrm{E}}-01$ & $2.33 \mathrm{E}+01$ & $5.00 \mathrm{E}-01$ & $7.33 \mathrm{E}+02$ & & & $6.94 \mathrm{E}-02$ \\
\hline Methyl Metacrylate & $4.50 \mathrm{E}-01$ & $1.57 \mathrm{E}+01$ & $\$ .4 \mathrm{E}+00$ & $7.33 \mathrm{E}+02$ & & & $6.02 \mathrm{E}-02$ \\
\hline $\begin{array}{l}\text { Cumene } \\
\text { (lsopropylbenzene) }\end{array}$ & $7.71 \mathrm{E}-03$ & $2.68 \mathrm{E}-01$ & $1.00 \mathrm{E}-01$ & $7.33 \mathrm{E}+02$ & & & $1.04 \mathrm{E}-03$ \\
\hline $\begin{array}{l}\text { (1-Methyl- } \\
\text { propyl)benzene }\end{array}$ & $7.71 \mathrm{E}-03$ & $2.6 \overline{\mathrm{SE}-01}$ & $3.70 \mathrm{E}-02$ & $7.33 \mathrm{E}+02$ & & & $1.04 \mathrm{E}-03$ \\
\hline 1,2-Dimethy]-benzene & $2.78 \mathrm{E}-02$ & $9.69 \mathrm{E}-01$ & $2.00 \mathrm{E}+00$ & $7.33 \mathrm{E}+02$ & & & $3.74 \mathrm{E}-04$ \\
\hline $\begin{array}{l}\text { 1-Methyl-4-(1- } \\
\text { ( methylethyl)benzene }\end{array}$ & $7.71 \mathrm{E}-03$ & $2 . \overline{68 E}-01$ & $3.70 \mathrm{E}-02$ & $7.33 \mathrm{E}+02$ & & & $1.04 \mathrm{E}-03$ \\
\hline Propylbenzene & $7.71 \mathrm{E}-03$ & $2.69 \mathrm{E}-01$ & $3.70 \mathrm{E}-02$ & $7.33 \mathrm{E}+02$ & & & $1.04 \mathrm{E}-03$ \\
\hline Bromodichloro-methane & 5.11E-0] & $1.78 \mathrm{E}+01$ & $2.00 \mathrm{E}-02$ & $7.33 \mathrm{E}+02$ & $6.20 \mathrm{E}-02$ & I.81E-03 & $5,36 \mathrm{E}-02$ \\
\hline Bromoform & $2.59 \mathrm{E}-0 \mathrm{I}$ & $9.05 e+00$ & $2.00 \mathrm{E}-02$ & $7.33 \mathrm{E}+02$ & $7.90 \mathrm{E}-03$ & $1.17 \mathrm{E}-04$ & 2.69E-02 \\
\hline Bromomethanc & $5.7 \overline{\mathrm{E}-01}$ & $2.00 \mathrm{E}+01$ & $1.40 \mathrm{E}-03$ & $7.33 \mathrm{E}+02$ & & & $6.82 \mathrm{E}-02$ \\
\hline Carbon Disulfide & $\mathrm{L} . \overline{99 \mathrm{E}-0 \mathrm{I}}$ & $6.94 \mathrm{E}+00$ & $1.00 \mathrm{E}-01$ & $7.33 \mathrm{E}+02$ & & & $2.66 \mathrm{E}-02$ \\
\hline Chloromelhane & $5.73 \mathrm{E}-01$ & $2.00 \mathrm{E}+01$ & & $7.33 E+02$ & $1.30 \mathrm{E}-02$ & $4.27 \bar{E}-04$ & \\
\hline o-Chlorotoluene & $6.31 \bar{E}-02$ & $2.20 \mathrm{E}+00$ & $2.00 \mathrm{E}-02$ & $7.33 \mathrm{E}+02$ & & & $2.44 \mathrm{E}-03$ \\
\hline Dibromochloromethane & $3.56 \mathrm{E}-01$ & $1,24 \mathrm{E}+01$ & $2.00 \mathrm{E}-02$ & $7.33 \mathrm{E}+02$ & $8.40 \mathrm{E}-02$ & $1.33 \mathrm{E}-03$ & $4.75 \mathrm{E}-02$ \\
\hline Dichlorodifluoromethane & $4.72 \mathrm{E}-02$ & $1.65 \mathrm{E}+00$ & $2.00 \mathrm{E}-0 \mathrm{I}$ & $7.33 \mathrm{E}+02$ & & & $6.30 \mathrm{E}-03$ \\
\hline 1,2,-cis-Dichloroethylene & $9.19 \mathrm{E}-02$ & $3.2 \mathrm{tE}+00$ & $1.00 \overline{\mathrm{E}-02}$ & $7.33 \mathrm{E}+02$ & & & $1.23 \mathrm{E}-03$ \\
\hline 1,2-trans-Dichloroethylene & $4.56 \mathrm{E}-01$ & $1.59 \mathrm{E}+0 \mathrm{I}$ & $2.00 \mathrm{E}-02$ & $7.33 \mathrm{E}+02$ & & & $6.09 \mathrm{E}-02$ \\
\hline 1,2-Dichloropropaje & $4.2 \overline{\mathrm{E}}-01$ & $1.49 \mathrm{E}+01$ & & $7.33 \mathrm{E}+02$ & $6.80 \mathrm{E}-02$ & $6.24 \mathrm{E}-06$ & \\
\hline
\end{tabular}


Tabte 2. Peak Effective Risks and Doses for the EMWMF for Hazardous Constituents (based on a $1 \mathrm{~kg} / \mathrm{m}^{3}$ concentration in the waste)

\begin{tabular}{|c|c|c|c|c|c|c|c|}
\hline Constituent & $\begin{array}{c}\text { Peak } \\
\text { Dose } \\
\text { in Bear } \\
\text { Creek } \\
(\mathrm{mg} / \mathrm{kg}- \\
\text { day) }\end{array}$ & $\begin{array}{c}\text { Peak } \\
\text { Concentration } \\
\text { in Bear Creek } \\
(\mathrm{mg} / \mathrm{L}) \\
\end{array}$ & $\begin{array}{c}\text { Reference } \\
\text { Dose } \\
\text { (mg/kg-day) }\end{array}$ & $\begin{array}{c}\text { Equivalent } \\
\text { Uptake } \\
\text { (U/yr) }\end{array}$ & $\begin{array}{c}\text { Slope } \\
\text { Factor } \\
(1 / \mathrm{mg} / \mathrm{kg}-\mathrm{d})\end{array}$ & $\begin{array}{c}\text { Peak } \\
\text { Effective } \\
\text { Risk } \\
\text { (ILCR) }\end{array}$ & $\begin{array}{c}\text { Peak } \\
\text { Effective } \\
\text { Dose } \\
\text { (mg/kg- } \\
\text { day) }\end{array}$ \\
\hline Ethylbenzene & $2.85 \mathrm{E}-02$ & $9.94 \mathrm{E}-01$ & $1.00 \mathrm{E}-01$ & $7.33 \mathrm{E}+02$ & & & $3.81 \mathrm{E}-03$ \\
\hline N-Hexine & $1.61 \mathrm{E}-03$ & $5.39 \mathrm{E}+01$ & $6.00 \mathrm{E}-02$ & $7.34 \mathrm{E}+02$ & & & $2.17 \mathrm{E}-04$ \\
\hline Methyl Isobutyl Ketone & $6.58 \mathrm{E}-01$ & $2.29 \mathrm{E}+01$ & $8.00 \mathrm{E}-02$ & $7.33 \mathrm{E}+02$ & & & $8.79 \mathrm{E}-02$ \\
\hline Propylene Glycol & $6.70 \mathrm{E}-01$ & $2.33 \mathbf{E}+01$ & $5.00 \mathrm{E}-0 \mathrm{l}$ & $7,33 \mathrm{E}+02$ & & & $8.99 \mathrm{E}-02$ \\
\hline Styrene & $5.23 \mathrm{E}-02$ & $1.82 \mathrm{E}+00$ & $2.00 \mathrm{E}-0 \mathrm{l}$ & $7.33 \mathbf{E}+02$ & & & $6.99 \mathrm{E}-03$ \\
\hline l,1,1,2-Tetrachloroethane & $1.8 \mathrm{IE}-01$ & $6.29 E+00$ & $3.00 \mathrm{E}-02$ & $7.33 \mathrm{E}+02$ & $2.60 \mathrm{E}-02$ & $2.76 \mathrm{E}-04$ & $1.89 \mathrm{E}-02$ \\
\hline 1,1,2,2-Tetrachloroethane & $1 . \overline{86 \mathrm{E}-01}$ & $1.18 E+01$ & $6.00 \mathrm{E}-02$ & $7.33 \mathrm{E}+02$ & $2,00 \mathrm{E}-01$ & $3.38 \mathrm{E}-03$ & $3.49 \mathrm{E}-03$ \\
\hline Trichlorofluoromethane & $1.86 \mathrm{E}-01$ & $6.47 \mathrm{E}+00$ & $3.00 \mathrm{E}-01$ & $7.33 \mathrm{E}+02$ & & & $2.48 \mathrm{E}-02$ \\
\hline 1,2,3-Trichtoropropane & $2.98 \mathrm{E}-01$ & $1.03 \mathrm{E}+01$ & $6.00 \mathrm{E}-03$ & $7.40 \mathrm{E}+02$ & $7.00 \mathrm{E}+00$ & [.27E-01 & $2,48 \mathrm{E}-02$ \\
\hline 1,2,4-Trimethylbenzene & $7.2 \mathrm{JE}-03$ & $2.51 \mathrm{E}-01$ & $5.00 \mathrm{E}-02$ & $7.33 \mathbf{E}+02$ & & & $9.71 \mathrm{E}-04$ \\
\hline 1,3,5-Trimethylbenzene & $8.14 \mathrm{E}-03$ & $2.84 \mathrm{E}-0 \mathrm{l}$ & $5.00 \mathrm{E}-02$ & $7.33 \mathrm{E}+02$ & & & $1.09 \mathrm{E}-03$ \\
\hline
\end{tabular}

Note: The ratio of the well dilution factor to the creck dilution factor is 0.13 .

* = Constituent not projected to appear within lov,000 ycars

lLCR $=$ incremental lifet ime cancer risk

${ }^{*}=$ The PATHRAE-Haz analysis dows not consider biodegradation or radivactive decay and, hence. is conservative. 
Table 3. Projected Peak Risks for EMWMF for Radioactive Constituents (risks based or contaminant concentrations in the waste equal to the current WAC)

\begin{tabular}{|c|c|c|c|}
\hline Nuclide & $\begin{array}{c}\text { Projected } \\
\text { Peak Risk } \\
\text { (ILCR) }\end{array}$ & $\begin{array}{l}\text { Risk } \\
\text { Criterion } \\
\text { (ILCR) }\end{array}$ & $\begin{array}{c}\text { Time of } \\
\text { Projected Peak Risk } \\
\text { (yr) }\end{array}$ \\
\hline $\mathrm{H}-3$ & I. $16 \mathrm{E}-1 \mid$ & $1.00 \mathrm{E}-05$ & 330 \\
\hline $\mathrm{C}-14$ & $5.59 \mathrm{E}-06$ & $1.00 \mathrm{E}-05$ & 380 \\
\hline Tc-99 & $2.36 \mathrm{E}-06$ & $1.00 \mathrm{E}-05$ & 410 \\
\hline$I-129$ & $5.17 \mathrm{E}-06^{*}$ & $1,00 \mathrm{E}-05$ & 900 \\
\hline $1 \mathrm{~J}-2,33$ & $2,17 \mathrm{E}-05$ & $1.00 E-04$ & 45,000 \\
\hline 1.234 & 2.3IE-05 & I.0OE -04 & 45,000 \\
\hline $4-235$ & $2.45 \mathrm{E}-05$ & $1.00 \mathrm{E}-04$ & 53,000 \\
\hline $\mathrm{U}-236$ & $2.49 \mathrm{E}-05$ & $100 \mathrm{E}-04$ & 46,000 \\
\hline U-238 & 2.59E-05 & $1.00 \mathrm{E}-04$ & 53,000 \\
\hline $\mathrm{Np}-237$ & $2.19 \mathrm{E}-05$ & I.00E-04 & 100,000 \\
\hline $\mathrm{Pu}-239$ & $3.27 \mathrm{E}-06$ & $1.00 \mathrm{E}-04$ & 95,000 \\
\hline $\mathrm{Pu}-240$ & $1.92 \mathrm{E}-08$ & $1.00 \mathrm{E}-04$ & 92,000 \\
\hline Am-241 & a & $\mathbf{a}$ & a \\
\hline
\end{tabular}

* = Bused on an I 129 WAC of $2.9 \mathrm{pCi}_{\mathrm{g}}$

$a=$ The PATHRAE code did not indicate a non-zero value for the concentration of Am-24I at any time before 100.000 years. The Am-24I nuclide has the same $\mathrm{K}_{\mathrm{d}} \mathrm{s}$ as the Pu-24I nuclide so it should arrive at key locations at the same time. However, since Am-24I has a half - life of 432 years, less than one tenth of thal for Pu-240, much more of it will decay away before ahout 84.000 years, the time al which Pu-240 begins to show up in a discemable concentration. Therefore it is concluded that the WAC for Am-24] should be set at the specific activity of that nuclide.

ILCR = incremental lifecime cancer risk 
Table 4. Projected Peak Risks and Doses for EMWMF for Hazardous Constituents (risks and doses based on contaminant concentrations in the waste equal to the corresponding current WAC)

\begin{tabular}{|c|c|c|c|c|c|}
\hline Contaminant & $\begin{array}{l}\text { Time of } \\
\text { Projected } \\
\text { Peak (yt) }\end{array}$ & $\begin{array}{c}\text { Projected } \\
\text { Peak Risk } \\
\text { (ILCR) }\end{array}$ & $\begin{array}{l}\text { Risk Criterion } \\
\text { (ILCR) }\end{array}$ & $\begin{array}{c}\text { Projected } \\
\text { Peak Dose } \\
\text { (mg/kg- } \\
\text { day) }\end{array}$ & $\begin{array}{c}\text { Dose } \\
\text { Criterion } \\
\text { (mg/kg-day) }\end{array}$ \\
\hline Acenaphthone & 251,000 & $*$ & * & $*$ & * \\
\hline Acenaphthylene & 34,000 & & & $1.72 \mathrm{E}-01$ & $1.8 \mathrm{E}-01$ \\
\hline Acetone & 600 & & & $2.29 \mathrm{E}-02$ & $1.0 \mathrm{E}-01$ \\
\hline Aldrin & 265,000 & $*$ & $*$ & $*$ & 4 \\
\hline Antimony & 5,200 & & & $1.41 \mathrm{E}-04$ & $1.2 \mathrm{E}-03$ \\
\hline Aroclor 1221 & 665,000 & س & $*$ & $*$ & $*$ \\
\hline Aroclos 1232 & $1,000,000$ & $2.21 \mathrm{E}-06$ & $1.0 \mathrm{E}-05$ & & \\
\hline Barium & 150,000 & $*$ & $*$ & $*$ & $*$ \\
\hline Boron & 10,000 & & & $3.69 \mathrm{E}-01$ & $600 \mathrm{E}-01$ \\
\hline Alpha-BHC & 12,000 & $2.29 \mathrm{E}-05$ & $1.0 \mathrm{E} 04$ & & \\
\hline Betil-BHC & 14,000 & $2.38 \mathrm{E}-05$ & $1.0 \mathrm{E}-04$ & & \\
\hline Delta-BHC & 14,000 & $2.33 \mathrm{E}-05$ & $1.0 \mathrm{E}-04$ & & \\
\hline Benzene & 4,800 & 2.37E-05 & $1,0 \mathrm{E}-04$ & & \\
\hline Benzoic Acid & 470 & & & $9.02 \mathbf{E}-01$ & $4.0 \mathrm{E}+00$ \\
\hline Benzyl Alcohol & 570 & & & $1.08 \mathrm{E}-01$ & $3.0 \mathrm{E}-01$ \\
\hline Carbazole & 23,000 & $4.00 \mathrm{E}-05$ & $1,0 \mathrm{E}-04$ & & \\
\hline Carbon Tetrachloride & 6,100 & $3.39 \mathrm{E}-05$ & $1.0 \mathrm{E}-04$ & $4.75 \mathrm{E}-04$ & $2.1 \mathrm{E}-03$ \\
\hline Chlorobenzene & 1,900 & & & $4.44 \mathrm{E}-03$ & $6.0 \mathrm{E}-02$ \\
\hline Chlordane & 280,000 & $*$ & * & $* *$ & $*$ \\
\hline Chloroform & 2,000 & $2.28 \mathrm{E}-06$ & $1.0 \mathrm{E}-04$ & $2.31 \mathrm{E}-04$ & $3.0 \mathrm{E}-02$ \\
\hline Chromium III & 33,000 & & & $3.69 \mathrm{E}-01$ & $3,0 \mathrm{E}+00$ \\
\hline m-Cresol & 780 & & & $1.14 \mathrm{E}-02$ & $5.0 \mathrm{E}-02$ \\
\hline 0 -Cresol & 1.100 & & & $1.16 \mathrm{E}-02$ & $5.0 \mathrm{E}-02$ \\
\hline p-Cresol & 770 & & & $1,16 \mathrm{E}-02$ & $5.0 \mathrm{E}-02$ \\
\hline Cyanide & 33,000 & & & $1.13 \mathrm{E}-02$ & $6.0 \mathrm{E}-02$ \\
\hline Dibenzla, hlanthracene & $>1,000,000$ & * & * & $*$ & $*$ \\
\hline 1-2-Dichlorobenzene & 2,900 & & & $2.03 \mathrm{E}-02$ & $2.7 \mathrm{E}-01$ \\
\hline 1-3-Dichlorobenzene & 44.000 & & & $6.19 \mathrm{E}-02$ & $2.7 \mathrm{E}-01$ \\
\hline 14-Dichlorobenzene & 4,500 & $2.26 \mathrm{E}-06$ & $10 \mathrm{E}-04$ & $6.90 \mathrm{E}-02$ & $9.0 \mathrm{E}-0 \mathrm{~J}$ \\
\hline Diethylphthalate & 1,300 & & & $8.80 \mathrm{E}-01$ & $80 \mathrm{E}-01$ \\
\hline Dimethylphthalate & $710^{\circ}$ & & & $2.26 \mathrm{E}-03$ & $1.0 \mathrm{E}+01$ \\
\hline Di-n-butyl-phthalate & 470 & & & $3.60 \mathrm{E}-02$ & $1.0 \mathrm{E}-01$ \\
\hline
\end{tabular}


Table 4. Projected Peak Risks and Doses for EMWMF for Hazardous Constituents (risks and doses based on contaminant concentrations in the waste equal to the corresponding current WAC)

\begin{tabular}{|c|c|c|c|c|c|}
\hline Contaninant & $\begin{array}{c}\text { Time of } \\
\text { Projected } \\
\text { Peak (yr) }\end{array}$ & $\begin{array}{l}\text { Projected } \\
\text { Peak Risk } \\
\text { (ILCR) }\end{array}$ & $\begin{array}{l}\text { Risk Criterion } \\
\text { (ILCR) }\end{array}$ & $\begin{array}{c}\text { Projected } \\
\text { Peak Dose } \\
\text { (mg/kg- } \\
\text { day) }\end{array}$ & $\begin{array}{c}\text { Dase } \\
\text { Criterion } \\
\text { (mg/kg-day) }\end{array}$ \\
\hline 2,4-Dinitrotoluene & 800 & $2.13 \mathrm{E}-06$ & $1.0 \mathrm{E}-05$ & $1.36 \mathrm{E}-04$ & $6.0 \mathrm{E}-03$ \\
\hline 2.6-Dinitrotoluene & $74]$ & $2.25 \mathrm{E}-06$ & $1.0 \mathrm{E}-05$ & $2.29 \mathrm{E}-04$ & $1.0 \mathrm{E}-03$ \\
\hline Dieldrin & 88,000 & $3.38 \mathrm{E}-05$ & I.OE-04 & $5.92 \mathrm{E}-05$ & $1.5 \mathrm{E}-04$ \\
\hline 2,4-D & 660 & & & $2.19 \mathrm{E}-04$ & $1.0 \mathrm{E}-02$ \\
\hline DDD & 250,000 & س & שس & $*$ & $*$ \\
\hline $\mathrm{DDE}$ & 6.100 & 4.01E-05 & $1.0 \mathrm{E}-04$ & & \\
\hline Endosulfan I & 14,000 & & & $3.67 \mathrm{E}-03$ & $1.8 \mathrm{E}-02$ \\
\hline Endosulfan II & 28,000 & & & $3.67 \mathrm{E}-03$ & $1.8 \mathrm{E}-02$ \\
\hline Endrin & 59,000 & & & $2.18 \mathrm{E}-04$ & $9.0 \mathrm{E}-04$ \\
\hline Endrin Aldehyde & 140,000 & & & $2.20 \mathrm{E}-04$ & $9.0 \mathrm{E}-04$ \\
\hline Endrin Kelone & 140,000 & & & $2.20 \mathrm{E}-04$ & $9.0 \mathrm{E}-04$ \\
\hline Hexachlorobenzene & 300,000 & 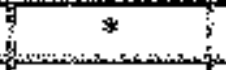 & $*$ & * & $*$ \\
\hline Hexachloroethane & 12,000 & $2.30 \mathrm{E}-05$ & $1.0 \mathrm{E}-04$ & $6.85 E+04$ & $3.0 \mathrm{E}-03$ \\
\hline Heptachlor & 130,000 & $2.38 \mathrm{E}-05$ & $1.0 \mathrm{E}-04$ & $3.53 \mathrm{E}-04$ & $1.5 \mathrm{E}-03$ \\
\hline Heptachlor Epoxide & 47,000 & $3.30 \mathrm{E}-05$ & $1.0 \mathrm{E}-04$ & $1.22 \mathrm{E}-05$ & $3.9 \mathrm{E}-05$ \\
\hline Lsophorone & 4,800 & $2.28 \mathrm{E}-05$ & $1,0 \mathrm{E}-04$ & I.37E-01 & $6.0 \mathrm{E}-01$ \\
\hline Lead & 540,000 & $*$ & $*$ & $\neq$ & $*$ \\
\hline Lindane & 23,000 & $2.21 \mathrm{E}-05$ & 1.0E-04 & $2.05 \mathrm{E}-04$ & $9.0 \mathrm{E}-04$ \\
\hline Manganese & $>1,000,000$ & $*$ & $*$ & $*$ & $\begin{array}{c}5 \\
\end{array}$ \\
\hline Molybdenum & 55,000 & & & $3.42 \mathrm{E}-03$ & $1.5 \mathrm{E}-02$ \\
\hline Methylene Chloride & 530 & $2.67 \mathrm{E}-07$ & $1.0 \mathrm{E}-05$ & $1.89 \mathrm{E}-02$ & $6.0 \mathrm{E}-02$ \\
\hline Methylcyclohexane & 470 & & & $2.94 \mathrm{E}-03$ & $1.3 \mathrm{E}-02$ \\
\hline 2-Methylnapthalene & 20,000 & & & $2.72 \mathrm{E}-03$ & $1.2 \mathrm{E}-02$ \\
\hline Nitrobenzene & 900 & & & $1.12 \mathrm{E}-04$ & $5.0 \mathrm{E}-04$ \\
\hline 4-Nitrobenzamine & 1,600 & 2.26E-06 & $1.0 \mathrm{E} \cdot 04$ & $3.69 \mathrm{E}-04$ & $9.0 \mathrm{E}-03$ \\
\hline $\begin{array}{l}\text { N-nitroso-di-n- } \\
\text { propolyamine }\end{array}$ & 2,300 & 2.11E-05 & $1,0 \mathrm{E}-04$ & & \\
\hline $\begin{array}{l}\mathrm{N}- \\
\text { Nitrosodjphenylamine }\end{array}$ & 2,600 & $2.18 \mathrm{E}-06$ & $1.0 \mathrm{E}-04$ & $4.53 \mathrm{E}-03$ & $6.0 \mathrm{E}-02$ \\
\hline Napthalene & 12,000 & & & $8.78 \mathrm{E}-03$ & $1.1 \mathrm{E}-01$ \\
\hline 2-Nitrophenol & 2,800 & & & $4.72 \mathrm{E}-05$ & $1.2 \mathrm{E}-01$ \\
\hline 4-Nittophenol & 3,300 & & & $8.78 \mathrm{E}-03$ & $12 \mathrm{E}-01$ \\
\hline Phenol & 2.200 & & & $1.24 \mathrm{E}-01$ & $6.0 \mathrm{E}-01$ \\
\hline
\end{tabular}


Table 4. Projected Peak Risks and Doses for EMWMF for Hazardous Constituents (risks and doses based on contaminant concentrations in the waste equal to the corresponding current WAC)

\begin{tabular}{|c|c|c|c|c|c|}
\hline Contaminant & $\begin{array}{l}\text { Time of } \\
\text { Projected } \\
\text { Peak (yr) }\end{array}$ & $\begin{array}{l}\text { Projected } \\
\text { Peak Risk } \\
\text { (ILCR) }\end{array}$ & $\begin{array}{l}\text { Risk Criterion } \\
\text { (ILCR) }\end{array}$ & $\begin{array}{l}\text { Projected } \\
\text { Peak Dose } \\
\text { (mg/kg- } \\
\text { day) }\end{array}$ & $\begin{array}{c}\text { Dose } \\
\text { Criterion } \\
\text { (mg/kg-day) }\end{array}$ \\
\hline Pyridine & 510 & & & $2.2 \mathrm{E}-04$ & 1.0E-03 \\
\hline Selenium & 81,000 & & & $1.2 \mathrm{JE}-02$ & $1.5 E-02$ \\
\hline 1 Strontium & 37,000 & & & $6.19 \mathrm{E}-01$ & $1.8 \mathrm{E}+00$ \\
\hline Tin & 14,000 & & & $6.69 \mathrm{E}-03$ & $1.8 \mathrm{E}+00$ \\
\hline Tetractlotoethene & 19,000 & $2.27 \mathrm{E}-05$ & $1.0 \mathrm{E}-04$ & $2.20 \mathrm{E}-02$ & $3.0 \mathrm{E}-02$ \\
\hline $\begin{array}{l}2,3,4,6- \\
\text { Tetrachlorophenol }\end{array}$ & 680,000 & * & $*$ & * & $*$ \\
\hline 12,4-Trichlorbenzene & 5,200 & & & $1.57 \mathrm{E}-03$ & $3.0 \mathrm{E}-02$ \\
\hline Trichloroethene & 7.100 & $2.30 \mathrm{E}-05$ & $1.0 \mathrm{E}-04$ & & \\
\hline $2,4,6$-Trichlorophenol & 2,500 & $2.25 \mathrm{E}-06$ & $1.0 \mathrm{E}-04$ & a & $3.0 \mathrm{E}-04$ \\
\hline Toluene & 16,000 & & & $1.35 \mathrm{E}-01$ & $6.0 \mathrm{E}-01$ \\
\hline 2,4,5-TP (Silvex) & 990 & 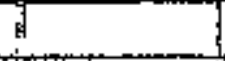 & & $1.80 \mathrm{E}-03$ & $8.0 \mathrm{E}-03$ \\
\hline $\mathrm{U}-233^{6}$ & 66,000 & & See Table 2 & $5.14 \mathrm{E}-04$ & $3,0 \mathrm{E}-03$ \\
\hline$U-234^{6}$ & 67,000 & & See Table 2 & $5.43 \mathrm{E}-04$ & $3.0 \mathrm{E}-03$ \\
\hline $0.235^{6}$ & 86,000 & & See Table 2 & $6.53 \mathrm{E}-04$ & $30 \mathrm{E}-03$ \\
\hline $\mathrm{U}-236^{\mathrm{b}}$ & 77,000 & & See Table 2 & $6.46 \mathrm{E}-04$ & $3.0 \mathrm{E}-03$ \\
\hline U-238 & 86,000 & & See Table 2 & $6.63 \mathrm{E}-04$ & $3.0 \mathrm{E}-03$ \\
\hline Vanadium & 540,000 & + & 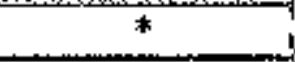 & & $*$ \\
\hline Vinyl Chloride & 1,700 & $5.61 \mathrm{E}-06$ & 1.0E-04 & $8.30 \mathrm{E}-05$ & $3.0 \mathrm{E}-03$ \\
\hline Xylene (mixture) & 3,400 & & & $4.29 \mathrm{E}-02$ & $6.0 \mathrm{E}-01$ \\
\hline Chloromethane & 560 & $2.99 \mathrm{E}-06$ & $1.0 \mathrm{E}-05$ & & \\
\hline Acetonitrile & 470 & & & $1.65 \mathrm{E}-04$ & $6.0 \mathrm{E}-03$ \\
\hline Acetophenone & 770 & & & $3.00 \mathrm{E}-02$ & $1.0 \mathrm{E}-01$ \\
\hline Acrolein & 470 & & & $7.38 \mathrm{E}-04$ & $5.0 \mathrm{E}-04$ \\
\hline Acrylonitrile & 480 & $3.04 \mathrm{E}-06$ & $10 \mathrm{E}-05$ & $2.96 \mathrm{E}-04$ & $1.0 \mathrm{E}-03$ \\
\hline Butylbenzene & 5,800 & & & $7.38 \mathrm{E}-04$ & \\
\hline Euhylchloride & 620 & $3.03 \mathrm{E}-06$ & 1.0E-05 & $1.20 \mathrm{E}-01$ & $4.0 \mathrm{E}-0 \mathrm{I}$ \\
\hline 1-Hexanol & 550 & & & $9,02 \mathrm{E}-03$ & $4.0 \mathrm{E}-02$ \\
\hline 2-Hexanone & 550 & & & $9.02 \mathrm{E}-03$ & $4.0 \mathrm{E}-02$ \\
\hline $\begin{array}{l}\text { Trimethylbenzene } \\
\text { (mixed isomers) }\end{array}$ & 5,200 & † & & $4.56 \mathrm{E}-02$ & $1.5 \mathrm{E}-01$ \\
\hline Dibenzofuran & 620,000 & $*$ & $*$ & 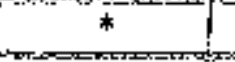 & ה \\
\hline 2,4-Dimethylphenol & 8.700 & & & $1.36 \mathrm{E}-02$ & $60 \mathrm{E}-02$ \\
\hline
\end{tabular}


Table 4. Projected Peak Risks and Doses for EMWMF for Hazardous Constituents (risks and doses based on contaminant concentrations in the waste equal to the corresponding current WAC)

\begin{tabular}{|c|c|c|c|c|c|}
\hline Contaminant & $\begin{array}{l}\text { Time of } \\
\text { Projected } \\
\text { Peak (yr) }\end{array}$ & $\begin{array}{l}\text { Projected } \\
\text { Peak Risk } \\
\text { (ILCR) }\end{array}$ & $\begin{array}{l}\text { Risk Criterion } \\
\text { (ILCR) }\end{array}$ & $\begin{array}{c}\text { Projected } \\
\text { Peak Dose } \\
\text { (mg/kg- } \\
\text { day) }\end{array}$ & $\begin{array}{c}\text { Dose } \\
\text { Criterion } \\
\text { (mg/kg-day) }\end{array}$ \\
\hline Benzidine & 18.000 & $3.79 \mathrm{E}-05$ & $1.0 \mathrm{E}-04$ & $1.91 \mathrm{E}-05$ & $6.0 \mathrm{E}-02$ \\
\hline Methanol & 410 & & & $1.22 \mathrm{E}-0 \mathrm{I}$ & $5,0 \mathrm{E}-01$ \\
\hline Methyl Metacrylate & 530 & & & $3.19 \mathrm{E}-01$ & $1.4 \mathrm{E}+00$ \\
\hline $\begin{array}{l}\text { Cumene } \\
\text { (Isopropyl-benzene) }\end{array}$ & 5,900 & & & $6.76 \mathrm{E}-02$ & $3.00 \mathrm{E}-1$ \\
\hline $\begin{array}{l}\text { (1-Methyl- } \\
\text { propyl)-benzene }\end{array}$ & 5,900 & 1 & & $2.48 \mathrm{E}-03$ & $1.1 \mathrm{E}-02$ \\
\hline 1,2-Dumethyl-benzene & 2,000 & & & $4.54 \mathrm{E}-02$ & $6.0 \mathrm{E}-02$ \\
\hline $\begin{array}{l}\text { 1-Methyl-4- } \\
\text { (1- melhylethyl)- } \\
\text { benzene }\end{array}$ & 5,900 & & & $2.48 \mathrm{E}-02$ & $1.1 \mathrm{E}-0 \mathrm{H}$ \\
\hline Propylbenzene & 5900 & & & $2.48 \mathrm{E}-02$ & $1.1 \mathrm{E}-01$ \\
\hline $\begin{array}{l}\text { Bromodichloro- } \\
\text { methane }\end{array}$ & 502 & $2.91 \mathrm{E}-06$ & $1.0 \mathrm{E}-05$ & $6.04 \mathrm{E}-03$ & $2.0 \mathrm{E}-02$ \\
\hline Bromoform & 1,300 & $3.01 \mathrm{E}-07$ & $1.0 \mathrm{E}-05$ & $2.64 \mathrm{E}-03$ & $2.0 \mathrm{E}-02$ \\
\hline Bromomethane & 560 & & & $4,30 \mathrm{E}-04$ & $1.4 \mathrm{E}-03$ \\
\hline Carbon Disulfide & 800 & & & $3.02 \mathrm{E}-02$ & $10 \mathrm{E}-01$ \\
\hline Chloromethane & $560^{\circ}$ & $2.99 \mathrm{E}-06$ & $1.0 \mathrm{E}-05$ & & \\
\hline o-Chlorotoluene & 3,300 & & & $1.20 \mathrm{E}-03$ & $2.0 \mathrm{E}-02$ \\
\hline $\begin{array}{l}\text { Dibromochloro- } \\
\text { methane }\end{array}$ & 930 & $7.80 \mathrm{E}-07$ & $1.0 \mathrm{E}-05$ & $5.58 \mathrm{E}-03$ & $2.0 \mathrm{E}-02$ \\
\hline $\begin{array}{l}\text { Dichlorodifluoro- } \\
\text { methane }\end{array}$ & 510 & & & $6.26 \mathrm{E}-02$ & $2,0 \mathrm{E}-0 \mathrm{I}$ \\
\hline $\begin{array}{l}\text { Dichloroethylene } \\
\text { Dichloris. }\end{array}$ & 3,700 & & & $9.80 \mathrm{E}-03$ & l.0E-0l \\
\hline $\begin{array}{l}\text { 1,2-trans- } \\
\text { Dichlotoetylene }\end{array}$ & 720 & & & $6.05 \mathrm{E}-03$ & $2.0 \mathrm{E}-02$ \\
\hline 1,2-Dichloropropane & 770 & $1,10 \mathrm{E}-08$ & $1.0 \mathrm{E}-05$ & & \\
\hline Ethylbenzene & 1,800 & & & $3.00 \mathrm{E}-02$ & $3.0 \mathrm{E}-01$ \\
\hline $\mathrm{N}$-Hexane & 1.400 & & & $6.13 \mathrm{E}-04$ & $6.0 \mathrm{E}-03$ \\
\hline Methyl Isobutyl Ketone & 480 & & & $2.39 \mathrm{E}-0 \mathrm{I}$ & $80 \mathrm{E}-0 \mathrm{I}$ \\
\hline Propylene Glycol & 470 & & & $1.58 \mathrm{E}-01$ & $1.25 \mathrm{E}+01$ \\
\hline Siyrene & 6,400 & & & $1.79 \mathrm{E}-01$ & $6.0 \mathrm{E}-01$ \\
\hline
\end{tabular}


Table 4. Projected Peak Risks and Doses for EMWMF for Hazardous Constituents (risks and doses based on contaminant concentrations in the waste equal to the corresponding current WAC)

\begin{tabular}{|c|c|c|c|c|c|}
\hline Contaminant & $\begin{array}{l}\text { Time of } \\
\text { Projected } \\
\text { Peak (yr) }\end{array}$ & $\begin{array}{l}\text { Projected } \\
\text { Peak Risk } \\
(\text { ILCR) }\end{array}$ & $\begin{array}{l}\text { Risk Criterion } \\
\text { (ILCR) }\end{array}$ & $\begin{array}{c}\text { Projected } \\
\text { Peak Dose } \\
\text { (mg/kg- } \\
\text { day) }\end{array}$ & $\begin{array}{c}\text { Dose } \\
\text { Criterion } \\
\text { (mg/kg-day) }\end{array}$ \\
\hline $\begin{array}{l}1,1,1,2- \\
\text { Tetrachloroethane }\end{array}$ & 1.500 & $3.10 \mathrm{E}-07$ & $1.0 \mathrm{E}-05$ & $8.89 \mathrm{E}-03$ & $3,0 \mathrm{E}-02$ \\
\hline $\begin{array}{l}1,1,2,2- \\
\text { Tetrachloroethane }\end{array}$ & 980 & $2.64 \mathrm{E}-06$ & $1.0 \mathrm{E}-05$ & $1.80 \mathrm{E}-03$ & $6.0 \mathrm{E}-03$ \\
\hline $\begin{array}{l}\text { Trichlorofluoro- } \\
\text { methane }\end{array}$ & 1,300 & & & $9.12 \mathrm{E}-02$ & $9.0 \mathrm{E}-01$ \\
\hline 1,2,3-Trichloropropane & 990 & $3.27 \mathrm{E}-06$ & $1.0 \mathrm{E}-05$ & $1.89 \mathrm{E}-03$ & $6.0 \mathrm{E}-03$ \\
\hline $\begin{array}{l}1,2,4- \\
\text { Trimethylbenzene }\end{array}$ & 5,200 & & & $3.39 \mathrm{E}-02$ & $1.5 \mathrm{E}-\mathrm{Ol}$ \\
\hline 1,3,5-Trimethylbenzene & 11,000 & & & $4.52 \mathrm{E}-02$ & $1.5 \mathrm{E}-01$ \\
\hline
\end{tabular}

$\mathbb{L C R}=$ incremental lifetime cancer risk

* = Constituent not projected to appear within 100,000 years.

$a=$ Both a slope factor and a reference dose exisı for this constituent but there is no dose-based WAC for it given in Revised Table A.1: Analyric WAC Limits. hitp://hechteljacobs, orafwebindex.htmil\#3.

"= The PATHRAE-Haz code does tolt sonsider biodegradation or radioactive decay and. hence, projected risk and dose estimates are conservative. 


\section{REFERENCES}

BJC 2003. Engineering Feasibility Plan for Groundwater Suppression at the Environmental Management Waste Management Facility, Oak Ridge, Tennessee, BJClOR-1478-RI. Oak Ridge, TN.

DOE 1998a. Remedial Investigation/Feasibility Study for the Disposal of Oak Ridge Reservation Comprehensive Environmental Response, Compensation, and Liability Act of 1980 Woste, DOE/OR02-1637 \& D2. Oak Ridge, TN.

DOE 1998b. Addendum to Remedial Investigation/Feasibility Study for the Disposat of Oak Ridge Reservation Comprehensive Environmental Response, Compensation, and Liability Acr of 1980 Waste. DOE/OR/02-1637 \& D2/A 1. Oak Ridge. TN.

DOE 200la. Attainment Plan for Risk/Toxicity-Based Waste Acceptance Criteria at the Oak Ridge Reservation. Oak Ridge, Tennessee, DOE/OR/0I-1909\&D3. Bechtel Jacobs Company LLC, Oak Ridge, TN.

DOE 2001b. Remedial Design Report for the Disposal of Oak Ridge Reservation Comprehensive Environmental Response, Compensation, and Liability Act of 1980 Waste, Oak Ridye. Tennessee, DOE/OR/01-1987\&D2, Oak Ridge, TN.

DOE 2010. Addendum in Remedial Design Report for the Disposal of Oak Ridge Reservation Comprehtensive Environmental Response. Compensation, and Liability Act of 1980 Waste, DOE/OR/1-1873/N18D2/A6. Oak Ridge, TN.

JEG 2010. Summary Report on the 2010 EMWMF Groundwater Modet and Flow/Fate-transport Arialyses. Jacobs Engineering, Oak Ridge, TN.

McDonald, M. G. and Harbaugh, B. W. 1988. A modular Three-Dimensional Finite-Difference Groundwater Flow Model. Book 6, Modeling Techniques, Chapter A1, U.S. Geological Survey, Reston, VirginiB.

Pollock, D. W, 1989. Documentation of Computer Programs to Compute and Disploy Pathines Using Results From the U.S. Geological Survey Modular Three-Dinensional FiniteDifference Groundwater Flow Model. U.S. Geological Survey Open-fïe Report 89-381.

RAE 1995a. The PATHRAE-RAD Perfomance Assessment Code for the Land Disposal of Radioactive Wastes, Rogers and Associates Engineering Corporation, RAE-9500/2-1. Salt Lake Cíty, UT.

RAE 1995b. The PATHRAE-HAZ Perfornance Assessment Code for the Land Disposal of Hazardous Chemical Wastes, Rogers and Associates Engineering Corporation, RAE9500/2-2, Salt Lake City, UT. 
Zheng, C. 1990. A Modular Three-Dimensional Transport Model for Simulation of Advection, Dispersion and Chemical Reactions of Contaminants in Groundwater Systems, S.S. Papadopulos \& Associates, Inc. 


\section{ATTACHMENT 1}

PATHRAE ENVIRONMENTAL TRANSPORT ANALYSIS INPUT AND OUTPUT FOR THE EMWMF

CELLS 1-6 
PATHRAE-RAD(PC) Version 2.2d February 1995

Date: 2-1-2010

Time: $13: 29: 52$

พ. A. C. - February 2010 New Proposed Cell 1-6 EMWMP

**** Mirror Image of Input Files $* * * * *$

-- Input File: ABCDEP. DAT

w. A. C. - February, 2010 New Proposed Cell 1-6 EMwhF $2,1000,100000$.

$35,0,5$

1,2

$0 ., 137 ., 788 ., 2.23 \mathrm{E}+5,1 ., 101,0$.

$1800.6,0 ., 0 ., 0 ., 0 ., 315,0$.

$20,2,0,1,1$

4. $0.15 .4,1.67 \mathbf{E}+06,-1,0 ., 1600 ., .40, .705,0.90,1$.

$1.0 \mathrm{E}=7,8000 \ldots 705,0 ., 1.0 \mathrm{E}+0,0.01,0 \mathrm{E}+0$

$240 ., 5.56 \mathrm{E}-4, .22, .02,3.0 \mathrm{E}-4,20 \ldots, 01$

$4,6.3,23,0 ., 1.1 \mathrm{E}-06, .01,0 ., 0,0,0,0$.

$0,0,0,0,0,0,0$

$1,0,0,1$

$0.0091,4.24,0.04,7.17, .025,10 ., 0.00001,1 \ldots, 0.25$

-- Input File: BRCDCF.DAT

$101, \mathrm{H}-3 \quad 6.4 \mathrm{E}-08, \quad 6.4 \mathrm{E}-08, \quad 0.00 \mathrm{E}+00$,

$102, \mathrm{C}-14$ 2.1E-06, 2.1E-06, 1.88E-09,

$103, \mathrm{~K}-40$ 1.9E-05, 1.2E-05, 1.4E-05

$104 . \mathrm{CO}^{-57}$ 1.2E-06, 9.1E-06, 1.34E-05,

105, Co-60 2.7E-05, 2.2E-04, 2.74E-04

$106, \operatorname{sr}-90 \quad 1.4 \mathrm{E}-04,1.3 \mathrm{E}-03,3.32 \mathrm{E}-08$,

$107, \mathrm{Nb}-95 \quad 2.6 \mathrm{E}-06,4.5 \mathrm{E}-06,7.8 \mathrm{E}-05$.

$108, \mathrm{Tc}-99$ 1.5E-06, 7.5E-06, 6.3E-11

$109, \mathrm{Cs}-134 \quad 7.3 \mathrm{E}-05,4.6 \mathrm{E}-05,1.78 \mathrm{E}-04$,

110, C5-137 5.0E-05, 3.2E-05, 6.85E-05, 


\begin{tabular}{|c|c|c|c|}
\hline 111 , Ba- 133 & 3. $4 \mathrm{E}-06$, & 6 - $9 \mathrm{E}-06$, & $4.2 \mathrm{E}-05$ \\
\hline 112, Eu- 152 & $6.5 E-06$, & 2. $2 E-04$, & $1.28 \mathrm{E}-04$ \\
\hline $113, E u-154$ & 9 - 5E- 06 , & $2.9 E=04$ & $1.39 \mathrm{E}-04$ \\
\hline 14. Eu-155 & 1. $5 E-06$. & 4. 1E-可, & $6-89 E-06$ r \\
\hline $55, \mathrm{Ra}-226$ & 8. 6E-03, & $7.9 \pm-03$. & $7.6 \mathrm{E}-07$. \\
\hline $16, \mathrm{Th}-22 \mathrm{~g}$ & 3. $8 \mathrm{E}-04$, & 3. $1 E-01$, & $2,8 \mathrm{E}-07$. \\
\hline $51, T h-229$ & $4.0 E-03$. & 2. OE+OO, & 1. . $0 E-05$, \\
\hline $36, T h-230$ & $5.5 E-04$ & $3.2 \mathrm{E}-01$, & 9.1E-0白, \\
\hline $37, \mathrm{Th}-232$ & $5.0 \mathrm{E}-03$, & $1.6 E+00$, & 6、44E一白， \\
\hline 53, Pa-231 & 2. $5 \mathrm{E}-02$. & $1.3 E+00$. & 3. $6 \mathrm{E}-06$, \\
\hline $21, \mathrm{U}-232$ & $1.31 \mathrm{E}-03$. & $1.4 \mathrm{E}-01$, & 白. $36 \mathrm{E}-0 \mathrm{~g}$, \\
\hline $54, U-233$ & $2.89 \mathrm{E}-04$ & 1. $4 \mathrm{E}-01$, & B, $36 \mathrm{E}-0 \mathrm{~g}$ \\
\hline $038, \mathrm{v}-234$ & $2.83 \mathrm{E}-04$. & 1. $3 \mathrm{E}-01$, & $8.74 \mathrm{E}-08$. \\
\hline $039, \mathrm{U}-235$ & $2.67 \mathrm{E}-04$. & 1. $2 \mathrm{E}-01$, & 1. $/ 3 \mathrm{E}-05$. \\
\hline $40, U-236$ & $2.69 \mathrm{E}-04$ & 1. $3 \mathrm{E}-01$, & $7.59 \mathrm{E}-08$, \\
\hline $041, \mathrm{U}-238$ & $2.7 \mathrm{E}-04$ & 1. $2 \mathrm{E}-01$, & $2.82 \mathrm{E}-06 \mathrm{~s}$ \\
\hline $042, \mathrm{~Np}-237$ & 4. $4 E-03$, & $4.9 \mathrm{E}-01$, & $3.2 \mathrm{E}-06 \mathrm{~s}$ \\
\hline $043, \mathrm{PU}-238$ & $3.2 \mathrm{E}-03$, & $3.90-01$. & $9.79 \mathrm{E}-08$, \\
\hline 044, Pu-2\$9 & $3.5 \mathrm{E}-03 x$ & 4. $3 \mathrm{E}-01$ & $4.29 \mathrm{E}-08 \mathrm{~s}$ \\
\hline $045, P \lambda=240$ & $3.5 \mathrm{E}-03$. & 5.1E-01s & $8.2 \mathrm{E}-08$ \\
\hline 048, $\mathrm{Am}-241$ & $3.6 \mathrm{E}-03$ & 4. $4 \mathrm{E}-01$, & $3.21 \mathrm{E}-06$ \\
\hline $132, \mathrm{Cm}-243$ & $2.5 \mathrm{E}-0.3$ & $3.1 \mathrm{E}-01$. & 1. $46 \mathrm{E}-05$. \\
\hline $050, \mathrm{Cm}-244$ & $2.05-03$ & 2. 5E-01, & $1.03 \mathrm{E}-07 \mathrm{~s}$ \\
\hline $020, I-129$ & $2.8 \mathrm{E}-04$ & 1. $8 \mathrm{E}-04$. & $2.2 \mathrm{E}-06$ \\
\hline $025, \mathrm{Be}=10$ & 4.2E-06. & $3.5 E-04$, & $0.0 \mathrm{E}-00$ \\
\hline
\end{tabular}

$\rightarrow$ Input File: INWNTRY.DAT

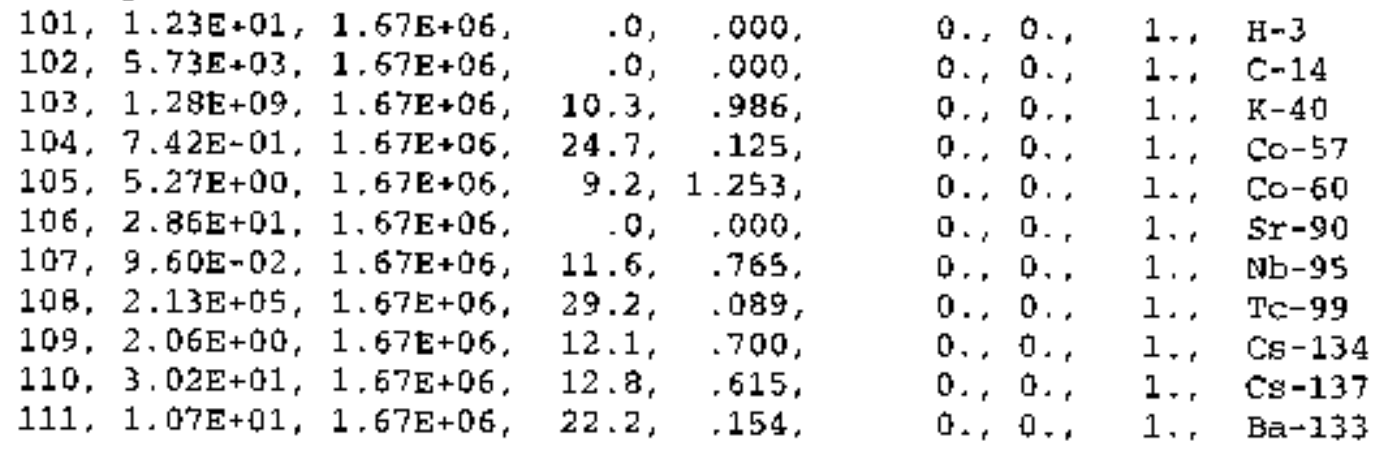




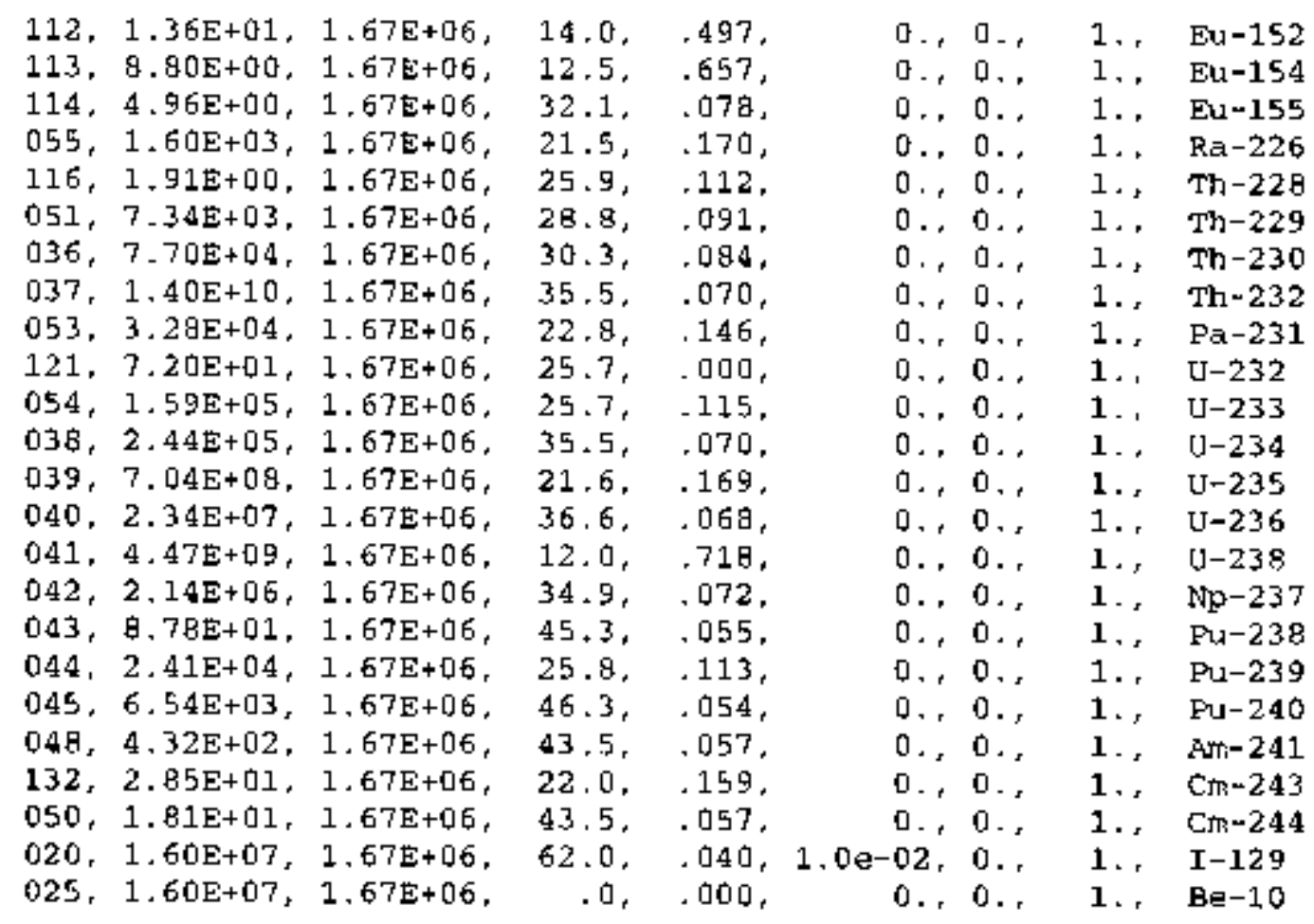

-- Input File: RQSITE. DAT

$101,-1,99 \mathrm{e}-1,0.00 \mathrm{E}+0,0.00 \mathrm{E}+0, \quad \mathrm{H}-3$

$102,-1.09 \mathrm{e}+0,0.00 \mathrm{E}+0,0.00 \mathrm{E}+0, \mathrm{C}-14$

$103,-3.98 \mathrm{e}+0,3.00 \mathrm{E}+0,3,00 \mathrm{E}+1, \mathrm{~K}-40$

$104,-3.97 \mathrm{e}+6,8.00 \mathrm{E}+1,8,00 \mathrm{E}+2, \mathrm{Co}-57$

$105,-3.97 e+6,8.00 \mathrm{E}+1,8.00 \mathrm{E}+2, \quad \mathrm{CO}-60$

$106,-8.74 \mathrm{e}+0,3.00 \mathrm{E}+0,3.00 \mathrm{E}+1, \mathrm{~S} r-90$

$107,-2.38 \mathrm{e}+1,3.79 \mathrm{E}+1,3.79 \mathrm{E}+2, \mathrm{Nb}-95$

108, $-1.29 \mathrm{e}+0,0.00 \mathrm{E}+0,0.00 \mathrm{E}+0, \mathrm{TC}-99$

$109,-1.99 \mathrm{e}+1,9.69 \mathrm{E}+1,9.69 \mathrm{E}+2, \quad \mathrm{Cs} \times 134$

$110,-1.99 \mathrm{e}+1,3.00 \mathrm{E}+2,3.00 \mathrm{E}+3, \mathrm{Cs}-137$

$111,-55.5 .50 \mathrm{E}+0,5.50 \mathrm{E}+1$, $\mathrm{Ba}-133$

$112,-3.78 \mathrm{e}+0,4.00 \mathrm{E}+0,4.00 \mathrm{E}+1, \mathrm{E}-252$ 
$113,-3.78 e+0,4,00 E+0,4.00 E+1, \quad E u-154$

$114,-3.79 e+0,4,00 E+0,4.00 E+1, \quad E u-155$

$055,-1.99 e+1,3,00 \mathrm{E}+2,3.00 \mathrm{E}+3, \mathrm{Ra}-226$

$116,-5,36 \mathrm{e}+1,3,00 \mathrm{E}+2,3.0 \mathrm{E}+3, \mathrm{Th}-22 \mathrm{~g}$

$051,-5.36 \mathrm{e}+1,3.00 \mathrm{E}+2,3.00 \mathrm{E}+3, \mathrm{Th}=229$

$036,-5,36 \mathrm{e}+1,3.00 \mathrm{E}+2,3,00 \mathrm{E}+3, \mathrm{Th}=230$

$037,-5,36 e+1,3,00 \mathrm{E}+2,3,0 \mathrm{E}+3, \mathrm{Th}-232$

$053,-5.47 \mathrm{e}+1,4,00 \mathrm{E}+1,4.00 \mathrm{E}+2, \mathrm{~Pa}-231$

$121,-4.00 \mathrm{e}+1,7,00 \mathrm{E}-1,2,00 \mathrm{E}+1, \mathrm{v}-232$

$054,-4.00 \mathrm{e}+1,7,00 \mathrm{E}-1,2,00 \mathrm{E}+1, \mathrm{D}-233$

$038,-4,00 \mathrm{e}+1,7,00 \mathrm{E}-1,2,00 \mathrm{E}+1, \quad \mathrm{D}-234$

$039,-4.00 \mathrm{e}+1,7,00 \mathrm{E}-1,2.00 \mathrm{E}+1, \mathrm{v}-235$

$040,-4,00 e+1,7.00 \mathrm{E}-1,2.00 \mathrm{E}+1, \mathrm{v}-236$

$041,-4.00 \mathrm{e}+1,7.00 \mathrm{E}-1,2.00 \mathrm{E}+1, \mathrm{v}-238$

$042,-5.56 \mathrm{E}+1,4.00 \mathrm{E}+0,4.00 \mathrm{E}+1, \quad \mathrm{~Np}-237$

$043,-5.76 e+1,4,00 E+0,4,00 E+1, \quad \mathbf{P u}-23 \mathrm{~g}$

$044,-5.76 e+1,4.00 E+0,4.00 E+1, \quad$ Pu-239

$045,-5.76 e+1,4.00 E+0,4.00 E+1, \quad P \downarrow-240$

$048,-5.76 e+1,4,00 E+0,4.00 E+1, \quad$ Am $=241$

$132,-5.76 e+1,4.00 \mathrm{E}+0,4.00 \mathrm{E}+1, \mathrm{Cm}-243$

$050,-5.76 \mathrm{e}+1,4.00 \mathrm{E}+0,4,00 \mathrm{E}+1, \mathrm{Cm}-244$

$020,-1.99 \mathrm{E}-1,0.00 \mathrm{E}+0,1.99 \mathrm{E}-1, \quad \mathrm{I}-129$

$025,-8,00 E+2,8,00 E+1,8.00 E+2, \quad \mathrm{Ee}-10$

-- Input File: UPTAKE, DAT

$0.5,0.2, \quad 1,89$

0.67 , 0.65, 2, $1 \mathrm{E}-3,439$,

$0.0,2160,24,21440,2,0,03$

$50.66,48, .480 .64$.

$.05,0.0008,60 \ldots, 0 ., 50$.

14.. $176.110 ., 0,95 \ldots, 730,6.9$

H-3 $25,4,8 \mathrm{E}+0,4,8 \mathrm{E}-1,1,0 \mathrm{E}-2,0 ., 1.2 \mathrm{E}-2,9.0 \mathrm{E}-1$

C-14 .25, 5.5E+1, 5.5E-1, 1.2E-2, 0., 3.1E-2, 4.6E+3

$\mathrm{K}-40 \quad .25,3.7 \mathrm{E}-1,3.7 \mathrm{E}-2,1,0 \mathrm{E}-2$,

Co-57 $25,9,4 \mathrm{E}-3,9,4 \mathrm{E}-4,1,0 \mathrm{E}-3$,

Co-60 .25, 9.4E-3, 9.4E-4, 1.0E-3,

$5 x-90 \quad .25,1.7 \mathrm{E}-2,1.7 \mathrm{E}-3,9,0 \mathrm{E}-4$,

$\mathrm{Nb}-95.25,9.4 \mathrm{E}-3,9,4 \mathrm{E}-4,2.5 \mathrm{E}-3$,

$0 ., 1.2 \mathrm{E}-2,1.0 \mathrm{E}+3$

0., $1.3 \mathrm{E}-2,5.0 \mathrm{E}+1$

$0 ., 1.3 \mathrm{E}-2,5.0 \mathrm{E}+1$

$0 ., 6.0 \mathrm{E}-4,3.0 \mathrm{E}+1$

$0 ., 2.8 \mathrm{E}-1,3.0 \mathrm{E}+4$ 
TC-99

$\mathrm{Cs}-134$

$\mathrm{Cs}-137$

$\mathrm{Ba}-133$

Eu-152

Eu-154

Eu-155

Ra-226

$\mathrm{Th}-228$

Th-229

Th -230

Th -232

Pa-231

$\mathrm{U}-232$

$\mathrm{U}-233$

$\mathrm{U}-234$

$\mathrm{U}-235$

(I) 236

$\mathrm{U}-238$

$\mathrm{Np}-237$

Pu-238

$\mathrm{P} y=239$

Fu-240

AIM-241

Cm-243

$\mathrm{Cm}-244$

I -129

Be-10
25, 2.5E-1, 2,5E-2, 1,0E-3,

$25,1,0 \mathrm{E}-2,1,0 \mathrm{E}-3,1,2 \mathrm{E}-2$

$25,1.0 \mathrm{E}-2,1,0 \mathrm{E}-3,1,2 \mathrm{E}-2$,

$25,5.0 \mathrm{E}-3,5.0 \mathrm{E}-4,4,0 \mathrm{E}-4$

$25,2.5 \mathrm{E}-3,2.5 \mathrm{E}-4,5.0 \mathrm{E}-6$

25, 2.5E-3, 2.5E-4, 5. $0 \mathrm{E}-6$,

.25, 2,5E-3, 2,5E-4, 5.0E-5,

$.25,3,1 \mathrm{E}-4,3-1 \mathrm{E}-5,9,0 \mathrm{E}-3$,

$25,4-2 \mathrm{E}-3,4,2 \mathrm{E}-4,5,0 \mathrm{E}-6$

$-25,4-2 \mathrm{E}-3,4,2 \mathrm{E}-4,5,0 \mathrm{E}-6$

$25,4.2 \mathrm{E}-3,4.2 \mathrm{E}-4,5.0 \mathrm{E}-6$

$25,4.2 \mathrm{E}-3,4.2 \mathrm{E}-4,5.0 \mathrm{E}-6$

$25,2.5 \mathrm{E}-3,2.5 \mathrm{E}-4,5,0 \mathrm{E}-6$,

$25,2,5 \mathrm{E}-3,2,5 \mathrm{E}-4,5,0 \mathrm{E}-4$,

$25,2.5 \mathrm{E}-3,2.5 \mathrm{E}-4,5.0 \mathrm{E}-4$,

$25,2.5 \mathrm{E}-3,2.5 \mathrm{E}-4,5.0 \mathrm{E}-4$

$25,2.5 \mathrm{E}-3,2.5 \mathrm{E}-4,5.0 \mathrm{E}-4$

25,2 . $5 \mathrm{E}-3,2,5 \mathrm{E}-4,5,0 \mathrm{E}-4$,

$25,2-5 \mathrm{E}-3,2,5 \mathrm{E}-4,5,0 \mathrm{E}-4$

$-25,2.5 \mathrm{E}-3,2,5 \mathrm{E}-4,5.0 \mathrm{E}-6$,

$25,2.5 \mathrm{E}-4,2.5 \mathrm{E}-5,2.0 \mathrm{E}-6$

$25,2.5 \mathrm{E}-4,2.5 \mathrm{E}-5,2.0 \mathrm{E}-6$

$25,2.5 \mathrm{E}-4,2+5 \mathrm{E}-5,2,0 \mathrm{E}-6$,

$25,2.5 \mathrm{E}-4,2.5 \mathrm{E}-5,5.0 \mathrm{E}-6$

$.25,2.5 \mathrm{E}-3,2.5 \mathrm{E}-4,5.0 \mathrm{E}-6$,

$25,2.5 \mathrm{E}-3,2.5 \mathrm{E}-4,5.0 \mathrm{E}-6$

$25,2.0 \mathrm{E}-2,2.0 \mathrm{E}-3,7.0 \mathrm{E}-3$.

$-25,1,0 E-2,2,5 E-3,9.0 E-7$,
0., $1.0 \mathrm{E}-4,1,5 \mathrm{E}+1$

$0.4 .0 \mathrm{E}-3,2.0 \mathrm{E}+3$

$0,4.0 \mathrm{E}-3,2.0 \mathrm{E}+3$

$0 ., 3.2 \mathrm{E}-3,4.0 \mathrm{E}+0$

$0 ., 4.8 \mathrm{E}-3,2.5 \mathrm{E}+1$

$0,4.6 \mathrm{E}-3,2.5 \mathrm{E}+1$

$0,4, \mathrm{BE}-3,2,5 \mathrm{~B}+1$

$0 ., 3.4 \mathrm{E}-2,5.0 \mathrm{E}+1$

$0,2.0 \mathrm{E}-4,3.0 \mathrm{E}+1$

0., 2. $0 \mathrm{E}-4,3,0 \mathrm{E}+1$

$0 ., 2.0 \mathrm{E}-4,3.0 \mathrm{E}+1$

$0 ., 2.0 \mathrm{E}-4,3.0 \mathrm{E}+1$

0. , 客, $0 \mathrm{E}+2,1,1 \mathrm{E}+1$

$0 ., 3,4 \mathrm{E}-4,2.0 \mathrm{E}+0$

$0 ., 3.4 \mathrm{E}-4,2.0 \mathrm{E}+0$

$0 ., 3.4 \mathrm{E}-4,2.0 \mathrm{E}+0$

$0 ., 3.4 \mathrm{E}-4,2.0 \mathrm{E}+0$

$0 ., 3,4 \mathrm{E}-4,2,0 \mathrm{E}+0$

0., 3, $\triangle \mathrm{E}-4,2,0 \mathrm{E}+0$

$0,2,0 \mathrm{E}-4,1.0 \mathrm{E}+1$

$0 ., 1.4 \mathrm{E}-5,3.5 \mathrm{E}+0$

$0 ., 1.4 \mathrm{E}-5,3.5 \mathrm{E}+0$

$0 ., 1,4 \mathrm{E}-5,3,5 \mathrm{E}+0$

$0 ., 2,0 \mathrm{E}-4,2,5 \mathrm{E}+1$

$0,2.0 \mathrm{E}-4,2.5 \mathrm{E}+1$

$0 ., 2.0 \mathrm{E}-4,2.5 \mathrm{E}+1$

$0 ., 1,0 \mathrm{E}-2,4,0 \mathrm{E}+1$

0., 1, 0E-2, 1., $\mathrm{E}+2$ 
TOTAL EQUIVALENT UPTAKE FACTORS FOR PATHRAE

\begin{tabular}{|c|c|c|c|c|c|c|}
\hline NUCLIDE & $\begin{array}{c}\text { UT (J, I) } \\
\text { RIVER } \\
\text { L/YR }\end{array}$ & $\begin{array}{l}\mathrm{UT}(\sqrt{ }, 2) \\
W E L L \\
\mathrm{~L} / \mathrm{YR}\end{array}$ & $\begin{array}{l}\mathrm{UT}(\mathrm{J}, 3) \\
\text { EROSION } \\
\text { L/YR }\end{array}$ & $\begin{array}{c}\text { UT }(J, 4) \\
\text { BATHTUB } \\
\text { L/YR }\end{array}$ & $\begin{array}{c}\text { UT (J, 5) } \\
\text { SPI LLAGE } \\
\text { L/YR }\end{array}$ & $\begin{array}{l}\text { UT (J, 6) } \\
\text { FOOD } \\
\text { KG/YR }\end{array}$ \\
\hline $\mathrm{H}-3$ & $.166 \mathrm{E}+03$ & $1.166 \mathrm{E}+03$ & $1.172 \mathrm{E}+03$ & $1.172 \mathrm{E}+03$ & $1.172 \mathrm{E} \bullet 03$ & $0.000 \mathrm{E}+00$ \\
\hline$c-14$ & $.564 \mathrm{E}+02$ & $9.564 \mathrm{E}+02$ & $3.270 \mathrm{E}+04$ & $3.270 \mathrm{E}+04$ & $3.270 \mathrm{E}+04$ & $0.000 \mathrm{E}+00$ \\
\hline $\mathrm{k}-40$ & $.639 \mathrm{E}+02$ & $8.639 \mathrm{E}+02$ & $7,764 E+03$ & $7.765 \mathrm{E}+03$ & $7.765 E+03$ & $1.308 E+01$ \\
\hline $\mathrm{Co}-57$ & $6.023 \mathrm{E}+02$ & 白. $023 \mathrm{E}+02$ & $1.146 \mathrm{E}+03$ & $1.146 \mathrm{E}+0 \mathrm{3}$ & $1.146 E+03$ & $1.933 \mathrm{E}=01$ \\
\hline$C 0-60$ & $.055 E+02$ & $B .055 \mathrm{E}+02$ & $1.150 \mathrm{E}+03$ & $1.150 \mathrm{E}+03$ & $1.150 E 403$ & $2.120 \mathrm{E}-01$ \\
\hline$S_{r}-90$ & $\Delta 14 \mathrm{E}+02$ & $7.414 \mathrm{E}+02$ & $9.484 \mathrm{E}+02$ & $9.484 E+02$ & $9.484 \mathrm{E} 402$ & $1.056 \mathrm{E}-01$ \\
\hline $\mathrm{Nb}-95$ & $.699 \mathrm{E}+03$ & $1.699 \mathrm{E}+03$ & 2. $007 \mathrm{E}+05$ & $2.007 \mathrm{E}+05$ & $2.007 E+05$ & $1.850 \mathrm{E}+00$ \\
\hline$T c-99$ & $8+02$ & $7.403 \mathrm{E}+02$ & $8.438 E+02$ & $8.43 .8 \mathrm{E}+02$ & $8.438 \mathrm{E}+02$ & $1.469 E+00$ \\
\hline$C s-134$ & $.365 \mathrm{E}+02$ & $8.365 E+02$ & $1.461 \mathrm{E}+04$ & $1.461 \mathrm{E}+04$ & $1.461 \mathrm{E}+04$ & $2.702 \mathrm{E}-01$ \\
\hline $\operatorname{cs} 137$ & . $371 \mathrm{E}+02$ & $8.371 E+02$ & $1.464 \mathrm{E}+04$ & 1. $464 \mathrm{E}+04$ & $1.464 E+04$ & $2.778 \mathrm{E}-01$ \\
\hline & +02 & $7,518 E+02$ & $7.794 \mathrm{E}+02$ & 7.79 & $9 \mathrm{E}+02$ & $4.490 \mathrm{E}-02$ \\
\hline Eu-152 & +02 & $7.572 \mathrm{E}+02$ & $9.296 E+02$ & 9.29 & $E+02$ & $2.623 E-02$ \\
\hline Eu-154 & $.571 E+02$ & $7.571 \mathrm{E}+02$ & $9.296 \mathrm{E}+02$ & 9.29 & $8+02$ & $2.614 \mathrm{E}-02$ \\
\hline$E(1-155$ & $7.570 \mathrm{E}+02$ & $7.570 \mathrm{E}+02$ & $9.294 \mathrm{E}+02$ & $9.294 \mathrm{E}+02$ & $9.294 \mathrm{E}+02$ & $2.595 \mathrm{E}-02$ \\
\hline & +02 & $7.337 E+02$ & $9.403 \mathrm{E}+02$ & $9.403 \mathrm{E}+02$ & $9.403 \mathrm{E}+02$ & $1.799 \mathrm{E}-02$ \\
\hline-2 & & 7.3 .8 & 7.51 & 7.51 & +02 & $1.356 \mathbf{E}=02$ \\
\hline $\mathrm{Cm}-243$ & $7,338 \mathrm{E}+02$ & $7.338 \mathrm{E}+02$ & $9.063 \mathrm{E}+02$ & $9.063 \mathrm{E}+02$ & 9.06 & $E-02$ \\
\hline $1-129$ & $8.327 \mathrm{E}+02$ & $8.327 \mathrm{E}+02$ & $1.109 \mathrm{E}+03$ & $1.109 \mathrm{E}+03$ & $1.109 \mathrm{E}+03$ & $5.624 \mathrm{E}-01$ \\
\hline Be-10 & 7.83 & $7.837 \mathrm{E}+02$ & $1.474 E+03$ & $1.474 \mathrm{E}+03$ & $1.474 E+03$ & $2.267 \mathrm{E}-0.1$ \\
\hline Th-230 & & $7,338 E+02$ & 9.40 & 9.41 & $E+02$ & $1.884 \mathrm{E}-02$ \\
\hline Th-232 & $.33 \mathrm{BE}+02$ & $7.338 \mathrm{E}+02$ & $9.406 \mathrm{E}+02$ & $9.410 \mathrm{E}+02$ & 9.405 & 1.8 \\
\hline $\mathrm{U}-234$ & $7,380 \mathrm{E}+02$ & $7.380 \mathrm{E}+02$ & $7.516 \mathrm{E}+02$ & $7,518 \mathrm{E}+02$ & $7.518 \mathrm{E}+02$ & $1.357 \mathrm{E}-02$ \\
\hline $\mathrm{v}-2$ & 7,38 & $7.380 \mathrm{E}+02$ & 7. $51 \mathrm{BE}+02$ & 7,51 & $7.518 \mathrm{E}+02$ & $1.357 \mathrm{E}-02$ \\
\hline $\mathrm{U}-2$ & 7.36 & $7.3 \mathrm{BOE}+02$ & $7,518 \mathrm{E}+02$ & 7.51 & $7.518 \mathrm{E}+02$ & $1.357 \mathrm{E}-02$ \\
\hline $\mathrm{U}-238$ & $. \exists 80 \mathrm{E}+02$ & $7,380 E+02$ & $7.518 \mathrm{E}+02$ & $7.518 \mathrm{E}+02$ & $3 E+02$ & $1.357 \mathrm{E}-02$ \\
\hline$N p-237$ & $\exists \exists B E+02$ & $7.338 \mathrm{E}+02$ & $8.028 \mathrm{E}+02$ & $8.028 E+02$ & $8.028 \mathrm{E}+02$ & $1.122 \mathrm{E}-02$ \\
\hline $\mathrm{Pu}-23 \mathrm{~B}$ & $7.329 E+02$ & $7.329 \mathrm{E}+02$ & $7.570 \mathrm{E}+02$ & $7.570 \mathrm{E}+02$ & $7.570 \mathrm{E}+02$ & $1.058 \mathrm{E}-03$ \\
\hline & $.329 E+02$ & $7,329 \mathrm{E}+02$ & $7.570 \mathrm{E}+02$ & $7.570 \mathrm{E}+02$ & $7.570 \mathrm{E}+02$ & $1.059 \mathrm{E}-03$ \\
\hline Fu -240 & 7. $329 \mathrm{E}+02$ & $7,329 \mathrm{E}+02$ & $7.570 \mathrm{E}+02$ & $7.570 \mathrm{E}+02$ & $7.570 \mathrm{E}+02$ & $1.059 \mathrm{E}-03$ \\
\hline & & $7.338 \mathrm{E}+02$ & $9.063 \mathrm{E}+02$ & $9.063 \mathrm{E}+02$ & $9.063 \mathrm{E}+02$ & $1.121 \mathrm{E}-03$ \\
\hline $\mathrm{C}_{\mathrm{m}}-244$ & $7.33 \mathrm{BE}+02$ & $7,338 \mathrm{E}+02$ & $9.063 \mathrm{E}+02$ & $9.063 \mathrm{E}+02$ & $9.063 \mathrm{E}+02$ & $1.116 \mathrm{E}-02$ \\
\hline Th-229 & $7.338 \mathrm{E}+02$ & $7.338 \mathrm{E}+02$ & $9.40 \mathrm{BE}+02$ & $9.408 \mathrm{E}+02$ & $9.409 \mathrm{E}+02$ & $1.884 \mathrm{E}-02$ \\
\hline
\end{tabular}


Pa-231 4. $476 \mathrm{E}+06 \quad 4.076 \mathrm{E}+06 \quad 4.076 \mathrm{E}+06 \quad 4.077 \mathrm{E}+06 \quad 4.079 \mathrm{E}+06 \quad 2.641 \mathrm{E}+03$

[-233 7.380E+02 $7.380 \mathrm{E}+02 \quad 7.518 \mathrm{E}+02 \quad 7.51 \mathrm{EE}+02 \quad 7.51 \mathrm{BE}+02 \quad 1.357 \mathrm{E}-02$

$\begin{array}{llllllll}\mathrm{R} a-226 & 9.620 \mathrm{E}+02 & 9.620 \mathrm{E}+02 & 1.307 \mathrm{E}+03 & 1.307 \mathrm{E}+03 & 1.307 \mathrm{E}+03 & 1.901 \mathrm{E}-02\end{array}$

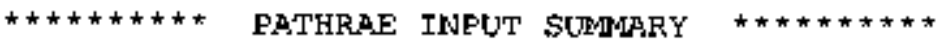

THERE ARE aO ISOTOPES IN THE DOSE FACTOR LIBRARY NUMBER OF TIMES FOR CALCULATION IS 2

YEARS TO BE CALCULATED ARE ...

1000.00100000 .00

THERE ARE 35 ISOTOPES IN THE INVENTORY FILE THE VALEE OF IFLAG IS 0 NUMBER OF PATHWAYS IS 5

PATHWAY
1 GROUNDWATER TO RIVER
$03 \mathrm{X}, \mathrm{I} 2,2 \mathrm{X}, \mathrm{A} 22,6 \mathrm{X}, \mathrm{J}, 2$,
$03 \mathrm{X}, \mathrm{I} 2,2 \mathrm{X}, \mathrm{A} 22,6 \mathrm{X}, \mathrm{I} 2$ !)
$0 \quad 3 \mathrm{X}, \mathrm{I2}, 2 \mathrm{X}, \mathrm{A22}, 6 \mathrm{X}, \mathrm{I} 2$ )
$0 \quad 3 \mathrm{X}, \mathrm{I2}, 2 \mathrm{X}, \mathrm{A} 22,6 \mathrm{X}, \mathrm{I} 2 \mathrm{\}}$,

TIME OF OPERATION OF WASTE FACILITY IN YEARS

LENGTH OF REPOSITORY (M)

WIITH OF REPOSITORY \{M\}

RIVER FLOW RATE (M**3/YR)

STREAM FLOW RATE (M**3/YR)

DISTANCE TO RIVER $\{M\}$

OPERATIONAL SPILLAGE FRACTION

DENSITY OF AQUIFER (KG/M** 3 \}

LONGITUDINAL DISPERSIVITY (M)

LATERAL DISPERSION COEFFICIENT -- Y AXIS $\left(\mathrm{M}^{*} * 2 / \mathrm{YR}\right)$

NUMBER OF MESH POINTS FOR DISPERSION CALCULATION

FLAG FOR GAMMA PATHWAY OFTIONS
TYPE OF USAGE

FOR UPTAKE FACTORS

2
0
0
0
0 
FLAG FOR GAMIMA BUILDUP CALCULATION

FLAG FOR ATMOSPHERIC PATHWAY

COVER THICKNESS OVER WASTE (M)

THICKNESS OF LASTSE IN PITS (M)

TOTAL INASTE VOLUME (M**3)

DISTANCE TO WELL - $\mathrm{x}$ COORDTNATE $\{\mathrm{M}$

DISTANCE TO WELL - Y COORDINATE (M)

DENSITY OF WASTE (KG/M**3)

FRACTION OF FOOD COWSUMED THAT IS GROWN ON SITE

FRACTION OF YEAR SPENT IN DIRECT RADIATION FIELD

DEPTH OF FLANT ROOT ZOHE (M)

AREAL DENSITY OF FLANTS (KG/M**2)

AVERAGE DUST LOADING IN AIR (KG/M**3)

ANNUAL ADULT BREATHING RATE (M**3/YR)

FRACTION OF YEAR EXPOSED TO DUST

CANISTER LIFETIME (YEARS)

INUENTORY SCAIING FACTOR

HEIGHT OF ROOMS IN RECLAIMER HOUSE (CM)

AIR CHANGE RATE IN RECLAIMER HOUSE (CHANGES/SEC)

RADON EMANATING POWER OF THE WASTE

DIFFUSION COEFF . OF RADON IN WASTE $(\mathrm{CM} * 2 / \mathrm{SEC})$

DIFFUSION COEFF, OF RN IN CONCRETE (CM**2/SEC)

THICKNESS OF CONCRETE SLAB FLOOR (CM)

DIFFUSION COEFF. OF RADON IN CONER (CM**2/SEC)

ATMOSPHERIC STAEILITY CLASS

AUERAGE WTND SPEED $\{\mathrm{M} / \mathrm{S}\}$

4.00

15.40

$1.670 \mathrm{E}+06$

-1 .

0.

1600 .

FRACTION OF TIME WIND BLOWS TOWARD RECEPTOR

RECEPTOR DISTANCE FOR ATMOSPHERIC PATHWAY (M)

DĐST RESUSFENSION RATE FOR OFFSITE TRANSPORT \{M*\#3/S\}

DEPOSITION VELOCITY $\{\mathrm{M} / \mathrm{S}\rangle$

STACK HEIGHT

STACK INSIDE DIAMETER \{M\}

$$
\begin{gathered}
.400 \\
.705 \\
.900 \\
1.000 \\
1.00 \mathrm{E}-07
\end{gathered}
$$

8000 .

.705

0 .

1. $, 0,0 \mathrm{E}+00$

240 .

$5.56 \mathrm{E}-0.4$

2. $20 \mathrm{E}-01$

2. DOE-02

3. $00 \mathrm{E}-04$

20,0

1. $00 \mathrm{E}-02$

4

6.30

.2300

.0

1. $10 \mathrm{E}-06$

0100

0

$D D$ 
STACK GAS VELOCITY $\{\mathrm{M} / \mathrm{S}\}$

HEAT EMISSION RATE FROM BURNING $\{C A L / S$ )

DECAY CHAIN FLAGS

FLAG FOR INPUT SUMMARY ERINTOUT

FLAG FOR DIRECTION OF TRENCH FILLING

FLAG FOR GROUNDWATER PATHWAY OPTIONS

AMOUNT OF WATER PERCOLATING THROUGH WASTE ANNUALLY (M)

DEGREE OF SOIL SATURATION

RESIDUAL SOIL SATURATION

PERMEABILITY OF VERTICAL ZONE (M/YR)

SOIL NUMEER

POROSITY OF AOUIFER

$\begin{array}{lllll}0 \quad 0 \quad 0 \quad 0 \quad 0 \quad 0 & 0.00 \mathrm{E}+00 \\ & & 0 \\ & & 0 \\ & & 0\end{array}$

POROSITY OF INSATURATED ZONE

DISTANCE FROM AQUIFER TO WASTE (M)

AVERAGE VERTICAL GROUNDWATER VELOCITY \{M/YR\}

$9.10 \mathrm{E}-03$

1.000

.000

.32

.000

04

LENGTH OF PERFORATED WELL CASING (M)

SURFACE. EROSION RATE (M/YR)

LEACH RATE SCALING FACTOR

ANNUAL RUNOFF OF ERECIPITATION (M)

.25

7.2

2.50E-02

4.2

10.000

$1.000 \mathrm{E}-05$

1.,000E+00

$0.00 \mathrm{E}+00$

***** PEAK CONCENTRATIONS AND TIMES FOR PATHNAY $1 * * * * *$

$* * * * *$ RIVER AT 101.0 M $\mathbf{M}^{* * * * *}$

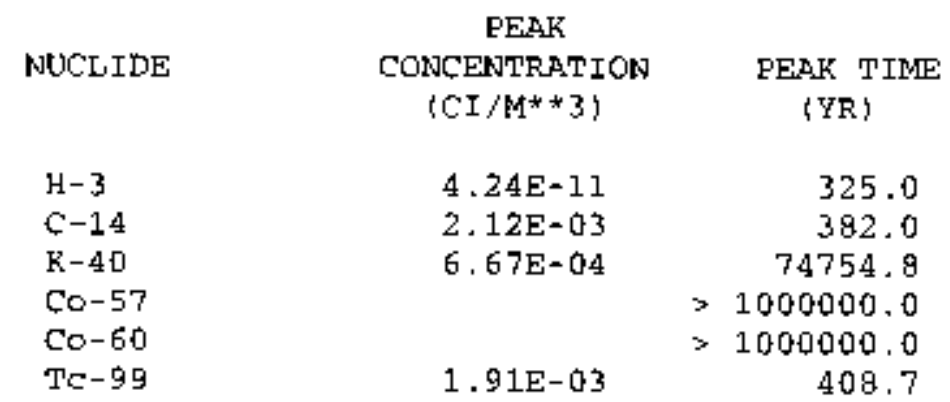

\section{AVERAGE DOSE \\ AT PEAK TIME \\ (MREM/YR)}

3. $16 \mathrm{E}-06$
4. $25 \mathrm{E}+03$
1. $10 \mathrm{E}+04$

4. $25 \mathrm{E}+03$

$2.12 \mathrm{E}+03$
AVERAGE RISK AT PEAK TIME

(HE/YR)

8. $855-13$

1. 19E-03

3. $07 \mathrm{E}-03$

TC-9

$5.94 \mathrm{E}-04$ 


$\begin{array}{ll}\mathrm{Cs}-134 & \\ \mathrm{Cs}-137 & \\ \mathrm{Th}-228 & 9.94 \mathrm{E}-04 \\ \mathrm{I}-129 & \\ \mathrm{Be}-10 & \\ \mathrm{Th}-230 & \\ \mathrm{Th}-232 & 6.06 \mathrm{E}-05 \\ \mathrm{U}-234 & 6.89 \mathrm{E}-05 \\ \mathrm{U}-235 & 6.99 \mathrm{E}-05 \\ \mathrm{U}-236 & 6.89 \mathrm{E}-05 \\ \mathrm{U}-238 & 4.80 \mathrm{E}-05 \\ \mathrm{~Np}-237 & 2.99 \mathrm{E}-06 \\ \mathrm{Pu}-239 & 2.19 \mathrm{E}-09 \\ \mathrm{Fu}-240 & \\ \mathrm{Th}-229 & 1.5 \mathrm{E}-13 \\ \mathrm{P}-231 & 5.66 \mathrm{E}-05 \\ \mathrm{~J}-233 & \\ \text { Ra-226 } & \end{array}$

31000000.0

1000000.0

$>1000000.0$

1000000,0

$>1000000,0$

$>1000000.0$ 447 自7.2 52983.0

46360.2 52983.0 101331.5

94921.3

92477,9

$>1000000.0$ 905476.8

44704.4

$>1000000.0$
$2.31 E+05 \quad 6.47 E-02$

$\begin{array}{ll}1.27 \mathrm{E}+04 & 3.54 \mathrm{E}-03 \\ 1.36 \mathrm{E}+04 & 3.80 \mathrm{E}-03 \\ 1.37 \mathrm{E}+04 & 3.82 \mathrm{E}-03 \\ 1.37 \mathrm{E}+04 & 3.84 \mathrm{E}-03 \\ 1.55 \mathrm{E}+05 & 4.34 \mathrm{E}-02 \\ 7.66 \mathrm{E}+03 & 2.14 \mathrm{E}-03 \\ 5.61 \mathrm{E}+00 & 1.57 \mathrm{E}-06 \\ & \\ 1.61 \mathrm{E}+01 & 4.52 \mathrm{E}-06 \\ 1.21 \mathrm{E}+04 & 3.38 \mathrm{E}-03\end{array}$

PATHWAY 1

GROUNDWATER TO RIVER

$* * * * * * * * *$ NUCLIDE DOSES (mrem/yr)

$\begin{array}{crr}\text { NUCLIDE/TIME } & 1000, & 100000 \\ \mathrm{H}-3 & 0.0 \mathrm{E}+00 & 0.0 \mathrm{E}+00 \\ \mathrm{C}-14 & 3.9 \mathrm{E}+03 & 0.0 \mathrm{E}+00 \\ \mathrm{~K}-40 & 0.0 \mathrm{E}+00 & 8.4 \mathrm{E}-07 \\ \mathrm{C}-57 & 0.0 \mathrm{E}+00 & 0.0 \mathrm{E}+00 \\ \mathrm{C}-60 & 0.0 \mathrm{E}+00 & 0.0 \mathrm{E}+00 \\ 5 \mathrm{~S}-90 & 0.0 \mathrm{E}+00 & 0.0 \mathrm{E}+00 \\ \mathrm{Nb}-95 & 0.0 \mathrm{E}+00 & 0.0 \mathrm{E}+00 \\ \mathrm{TC}-99 & 2.1 \mathrm{E}+03 & 0.0 \mathrm{E}+00 \\ \mathrm{Cs}-134 & 0.0 \mathrm{E}+00 & 0.0 \mathrm{E}+00\end{array}$




$\begin{array}{lll}\mathrm{Cs}-137 & 0.0 \mathrm{E}+00 & 0.0 \mathrm{E}+00 \\ \mathrm{Ea}-133 & 0.0 \mathrm{E}+00 & 0.0 \mathrm{E}+00 \\ \mathrm{E}-152 & 0.0 \mathrm{E}+00 & 0.0 \mathrm{E}+00 \\ \mathrm{E}-154 & 0.0 \mathrm{E}+00 & 0.0 \mathrm{E}+00 \\ \mathrm{Eu}-155 & 0.0 \mathrm{E}+00 & 0.0 \mathrm{E}+00 \\ \mathrm{Th}-228 & 0.0 \mathrm{E}+00 & 0.0 \mathrm{E}+00 \\ \mathrm{U}-232 & 0.0 \mathrm{E}+00 & 0.0 \mathrm{E}+00 \\ \mathrm{Cm}-243 & 0.0 \mathrm{E}+00 & 0.0 \mathrm{E}+00 \\ \mathrm{I}-129 & 2.3 \mathrm{E}+05 & 0.0 \mathrm{E}+00 \\ \mathrm{BE}-10 & 0.0 \mathrm{E}+00 & 0.0 \mathrm{E}+00 \\ \mathrm{Th}-230 & 0.0 \mathrm{E}+00 & 0.0 \mathrm{E}+00 \\ \mathrm{Th}-232 & 0.0 \mathrm{E}+00 & 0.0 \mathrm{E}+00 \\ \mathrm{U}-234 & 0.0 \mathrm{E}+00 & 1.1 \mathrm{E}+04 \\ \mathrm{U}-235 & 0.0 \mathrm{E}+00 & 1.4 \mathrm{E}+04 \\ \mathrm{U}-236 & 0.0 \mathrm{E}+00 & 1.4 \mathrm{E}+04 \\ \mathrm{U}-238 & 0.0 \mathrm{E}+00 & 1.4 \mathrm{E}+04 \\ \mathrm{~Np}-237 & 0.0 \mathrm{E}+00 & 1.5 \mathrm{E}+05 \\ \mathrm{Pu}-238 & 0.0 \mathrm{E}+00 & 0.0 \mathrm{E}+00 \\ \mathrm{Pu}-239 & 0.0 \mathrm{E}+00 & 6.9 \mathrm{E}+03 \\ \mathrm{Pu}-240 & 0.0 \mathrm{E}+00 & 3.1 \mathrm{E}+00 \\ \mathrm{~A} J-241 & 0.0 \mathrm{E}+00 & 0.0 \mathrm{E}+00 \\ \mathrm{Cm}-244 & 0.0 \mathrm{E}+00 & 0.0 \mathrm{E}+00 \\ \mathrm{Th}-229 & 0.0 \mathrm{E}+00 & 0.0 \mathrm{E}+00 \\ \mathrm{Ea}-231 & 0.0 \mathrm{E}+00 & 0.0 \mathrm{E}+00 \\ \mathrm{U}-233 & 0.0 \mathrm{E}+00 & 9.5 \mathrm{E}+03 \\ \mathrm{Rz}-226 & 0.0 \mathrm{E}+00 & 0.0 \mathrm{E}+00\end{array}$

NUCLIDE CONCENTRATIONS IN RIVER (Ci/m**3)

$\begin{array}{crr}\text { NUCLIDE/TIME } & 1000 . & 100000 \\ \mathrm{H}-3 & 0.0 \mathrm{E}+00 & 0.0 \mathrm{E}+00 \\ \mathrm{C}-14 & 2.0 \mathrm{E}-03 & 0.0 \mathrm{E}+00 \\ \mathrm{~K}-40 & 0.0 \mathrm{E}+00 & 5.1 \mathrm{E}-14 \\ \mathrm{C}-57 & 0.0 \mathrm{E}+00 & 0.0 \mathrm{E}+00 \\ \mathrm{C}-60 & 0.0 \mathrm{E}+00 & 0.0 \mathrm{E}+00 \\ \mathrm{~S}-90 & 0.0 \mathrm{E}+00 & 0.0 \mathrm{E}+00 \\ \mathrm{Nb}-95 & 0.0 \mathrm{E}+00 & 0.0 \mathrm{E}+00\end{array}$




\begin{tabular}{|c|c|c|}
\hline TC-99 & 1. $9 E-03$ & $0.0 \mathrm{E}+00$ \\
\hline$C 5-134$ & $0.0 E+00$ & $0.0 \mathrm{~B}+00$ \\
\hline Cs-137 & $0.0 \mathrm{E}+00$ & $0.0 E+00$ \\
\hline $6 a-133$ & $0.9 E+00$ & $0.0 E+00$ \\
\hline Eu-152 & $0.0 \mathrm{E}+00$ & $0.0 \mathrm{E}+00$ \\
\hline$E u-154$ & $0.0 \mathrm{E}+00$ & $0.0 \mathrm{E}+00$ \\
\hline Eu-155 & $0.0 \mathrm{E}+00$ & $0.0 \mathrm{E}+0.0$ \\
\hline Th-22g & $0.0 \mathrm{E}+0.0$ & $0.0 \mathrm{E}+00$ \\
\hline $\mathrm{v}-232$ & $0.0 \mathrm{E}+00$ & $0.0 E+00$ \\
\hline$c m-243$ & $0.0 \mathrm{E}+00$ & $0.0 \mathrm{E}+00$ \\
\hline$I-129$ & $9.8 E-04$ & $0.0 \mathrm{E}+00$ \\
\hline$B e-10$ & $0.0 \mathrm{E}+0.0$ & $0.0 \mathrm{E}+00$ \\
\hline$T K_{1}=230$ & $0.0 \mathrm{E}+00$ & $0.0 \mathrm{E}+0.0$ \\
\hline The -232 & $0.0 \mathrm{E}+00$ & $0.0 \mathrm{E}+00$ \\
\hline $\mathrm{v}-234$ & $0.0 \mathrm{E}+00$ & $5.2 \mathrm{E}-05$ \\
\hline$U-235$ & $0.0 E+00$ & $6.9 \mathrm{E}-05$ \\
\hline $\mathrm{U}-236$ & $0.0 E+00$ & $6.9 E-05$ \\
\hline $\mathrm{U}-23 \mathrm{~B}$ & $0.0 \mathrm{E}+00$ & $6.9 E-05$ \\
\hline $\mathrm{Np}-237$ & $0.0 \mathrm{E}+00$ & $4.8 E-05$ \\
\hline Pu-238 & $0.0 \mathrm{E}+00$ & $0.0 \mathrm{E}+00$ \\
\hline $\mathrm{Fu}-239$ & $0.0 \mathrm{E}+00$ & $2.7 \mathrm{E}-06$ \\
\hline$P x-240$ & $0.0 \mathrm{E}+00$ & $1,2 \mathrm{E}-09$ \\
\hline$A M-241$ & $0.0 \mathrm{E}+00$ & $0.0 \mathrm{E}+00$ \\
\hline Cm-244 & $0.0 \mathrm{E}+00$ & $0.0 \mathrm{E}+00$ \\
\hline Th-229 & $0.0 \mathrm{E}+00$ & $0.0 E+00$ \\
\hline $\mathrm{Pa}-231$ & $0.0 \mathrm{E}+00$ & $0.0 E+00$ \\
\hline $\mathrm{U}-233$ & $0.0 \mathrm{E}+00$ & $4.5 E-05$ \\
\hline Ra $a-226$ & $0.0 \mathrm{E}+00$ & $0.0 \mathrm{E}+00$ \\
\hline
\end{tabular}


PATHRAE-HAZ \{PC) Version 2.2A Pebruary 1995

Date: 2-1-2010

Time: $10: 36: 31$

W. A. C. - February, 2010 New Proposed Ce11 1-6 EMMMF

TOTAL EQUIVALENT UPTAKE FACTORS FOR PATHRAE

\begin{tabular}{|c|c|c|c|c|c|c|}
\hline CONTAMINANT & $\begin{array}{l}\text { UT \{J, 1\} } \\
\text { RIVER } \\
\mathrm{L} / \mathrm{YR}\end{array}$ & $\begin{array}{l}\text { UT }\{J, 2\} \\
\text { WELL } \\
\text { L/YR }\end{array}$ & $\begin{array}{l}\text { IT }\{J, 3\} \\
\text { EROSIOW } \\
\text { L/YR }\end{array}$ & $\begin{array}{l}\text { UT }\{(J, 4\} \\
\text { BATHTUB } \\
\text { L/YR }\end{array}$ & $\begin{array}{l}\text { UT ‘J , 5) } \\
\text { SPILIAGE } \\
\text { I/YR }\end{array}$ & $\begin{array}{l}\mathrm{UT}\{\mathrm{T}, 6\} \\
\mathrm{FOOD} \\
\mathrm{KG} / \mathrm{YR}\end{array}$ \\
\hline Acenaphthene & $.365 E+02$ & $7.365 E+02$ & $8.326 \mathrm{E}+03$ & B. $327 \mathrm{E}+03$ & $8.327 E+03$ & 6.1 \\
\hline Acetone & $7.32 \mathrm{gE}+02$ & $7.328 \mathrm{E}+02$ & 7. $32 \mathrm{BE}+02$ & $7.328 \mathrm{E}+02$ & $7.328 E+02$ & $5.480 \mathrm{E}+01$ \\
\hline Antimony & $.332 \mathrm{E}+02$ & $7.332 \mathrm{E}+02$ & $1.423 \mathrm{E}+03$ & $1.423 \mathrm{E}+03$ & 1. $423 \mathrm{E}+03$ & $2.153 \mathbf{E}-01$ \\
\hline Bärium & $+372 \mathrm{E}+02$ & $7.372 \mathrm{E}+02$ & $7.648 \mathrm{E}+02$ & $7.649 \mathrm{E}+02$ & $7.649 \mathrm{E}+02$ & $5.213 \mathrm{E}-01$ \\
\hline Bor & $.474 \mathrm{E}+02$ & $7.474 E+02$ & $7.474 \mathrm{E}+02$ & $7.477 \mathrm{E}+02$ & $7.477 \mathrm{E}+02$ & 3. $.26 \mathrm{E}+01$ \\
\hline $\mathrm{Al}$ & $7.342 \mathrm{E}+02$ & $7.342 \mathrm{E}+02$ & $7.342 \mathrm{E}+02$ & $7.343 \mathrm{E}+02$ & $7.343 E+02$ & $9.609 \mathrm{E}-01$ \\
\hline Bet & $6 \mathrm{E}+02$ & $7.346 \mathrm{E}+02$ & $7.346 \mathrm{E}+02$ & $7.345 \mathrm{E}+02$ & $7+346 \mathbf{E}+02$ & $8.399 \mathrm{E}-01$ \\
\hline & $.329 \mathrm{E}+02$ & $7.329 \mathrm{E}+02$ & $7.329 \mathrm{E}+02$ & $7.330 E+02$ & $7.3305+02$ & $3.82 \mathrm{DE}+\square 0$ \\
\hline Carbaz & $7.340 \mathrm{E}+02$ & $7.340 E+02$ & $3.839 \mathbf{E}+03$ & $3.839 \mathrm{E}+03$ & $3.939 E+03$ & 1. $081 E+\square 0$ \\
\hline Car & $.329 E+02$ & $7,329 \mathrm{E}+02$ & $7.329 E+02$ & $7.329 \mathrm{E}+02$ & $7.329 \mathrm{E}+02$ & $1.231 E+00$ \\
\hline orm & $.328 E+02$ & $7.328 \mathrm{E}+02$ & $7.328 E+02$ & $7.328 \mathrm{E}+02$ & $7.32 \mathrm{dE}+02$ & $2.955 E+00$ \\
\hline niџm III & $7.787 E+02$ & $7.787 \mathrm{E}+02$ & $2.159 E+03$ & $2.159 \mathrm{E}+03$ & $2.159 E+03$ & 6. $445 \mathrm{E}-01$ \\
\hline & $.286 \mathrm{E}+02$ & $8.286 \mathrm{E}+02$ & $8.284 E+02$ & $8.287 E+02$ & B. $287 \mathrm{E}+02$ & $2.459 E+00$ \\
\hline Flphthalat & $.061 E+02$ & $8.051 \mathbf{E}+02$ & $8.061 \mathbf{E}+02$ & $8.061 E+02$ & 日. $061 \mathrm{E}+02$ & 1. $249 \mathrm{E}-01$ \\
\hline ne & $7.329 \mathrm{E}+02$ & $7.329 E+02$ & $7.328 \mathrm{E}+02$ & $7.329 \mathrm{E}+02$ & $7.329 E+02$ & $2.030 \mathrm{E}+00$ \\
\hline Let & $-369 \mathbf{E}+02$ & $7.369 E+02$ & $2.807 E+03$ & $2.807 \mathrm{E}+03$ & $2.807 E+03$ & $4.682 E-01$ \\
\hline Ja & $.355 E+02$ & $7.355 \mathrm{E}+02$ & $3.496 E+03$ & $3+498 E+03$ & 3. $496 \mathrm{E}+03$ & $3.346 \mathrm{E}+00$ \\
\hline de & $.328 E+02$ & $7.329 E+02$ & $7.328 \mathrm{E}+\square 2$ & $7,328 \mathrm{E}+02$ & 7.32 白 +02 & $0.000 \mathrm{E}+00$ \\
\hline Nap & $7.332 \mathbf{E}+02$ & $7.332 \mathrm{E}+\mathrm{Cr} 2$ & Z. $044 E+03$ & $2.044 \mathrm{E}+03$ & $2.044 E+03$ & 1. $981 \mathrm{E}+00$ \\
\hline Npropy 1 & $7.328 E+02$ & $7.328 E+02$ & $7.797 \mathrm{E}+02$ & $7.797 E+02$ & $7.797 \mathrm{E}+02$ & $2.488 \mathrm{E}+01$ \\
\hline Phe & $7.328 \mathrm{E}+02$ & $7.328 \mathrm{E}+02$ & $7.887 \mathrm{E}+02$ & ᄀ. 8в7E+02 & $7.887 \mathrm{E}+02$ & $2.150 E+01$ \\
\hline Seleлium & $1.312 \mathrm{E}+03$ & $1.312 \mathrm{E}+03$ & $1.312 \mathrm{E}+03$ & $1.316 \mathrm{E}+03$ & $1.316 E+03$ & $7.577 \mathbf{E}+01$ \\
\hline & $7.941 E+02$ & $7.941 \mathrm{E}+02$ & $7.931 E+02$ & $7.941 \mathrm{E}+02$ & $7.94 \pm E+02$ & $2.096 E+01$ \\
\hline Tet & $7.329 E+02$ & $7.329 \mathrm{E}+02$ & $7.329 \mathrm{E}+02$ & $7.329 \mathrm{E}+02$ & $7.329 E+02$ & $1.273 \mathrm{E}+00$ \\
\hline Tin & $7.907 E+02$ & 7. $907 \mathrm{E}+02$ & $2.149 \mathrm{E}+04$ & $2.149 \mathrm{E}+04$ & $2.149 \mathrm{E}+04$ & $1.895 \mathrm{E}+01$ \\
\hline
\end{tabular}


Toluene

Trichloroethene

U-233

U-234

$\mathrm{U}-235$

$\mathrm{U}-2.36$

U-238

Varadium

$124 T r i c h l o r b$

Molybdenum,

berzidine

chlordane

Methylpropyle

124trimethy1日

MethylethylB

12DiketrylB

Propyle

Aldrin

Aroclor1221

Aroclor 1232

DDD

DDE

Acenaphthylene

Benzoic Acid

Dibenzofuran

Diethylphth

24-Dimethylphe

Dimethylphth

2Methylnaptha

2346 Tetrachlor

Benzyl Alcohol

Acentonitrile

Acrolien

Acylonitrle

Bromodichloro

Bromoform

Bromometh

carbondis
$7.330 \mathrm{E}+02 \quad 7.330 \mathrm{E}+02 \quad 7.329 \mathrm{E}+02 \quad 7.330 \mathrm{E}+02 \quad 7.330 \mathrm{E}+02 \quad 1.106 \mathrm{E}+00$ $7.329 \mathrm{E}+02 \quad 7.329 \mathrm{E}+02 \quad 7.329 \mathrm{E}+02 \quad 7.329 \mathrm{E}+02 \quad 7.329 \mathrm{E}+02 \quad 1.735 \mathrm{E}+00$ $7.371 \mathrm{E}+02 \quad 7.371 \mathrm{E}+02$ B. $061 \mathrm{E}+02 \quad 8.061 \mathrm{E}+02 \quad 8.061 \mathrm{E}+02 \quad 1.201 \mathrm{E}-01$ $7.371 \mathrm{E}+02 \quad 7.371 \mathrm{E}+02$ 月. $061 \mathrm{E}+02 \quad 8.061 \mathrm{E}+02 \quad 8.061 \mathrm{E}+02 \quad 1.201 \mathrm{E}-01$ $7.371 \mathrm{E}+02 \quad 7.371 \mathrm{E}+02 \quad 8.061 \mathrm{E}+02 \quad 8.061 \mathrm{E}+02 \quad 8.061 \mathrm{E}+02 \quad 1.201 \mathrm{E}-01$ $7,371 \mathrm{E}+02 \quad 7.371 \mathrm{E}+02 \quad 8.061 \mathrm{E}+02 \quad 8.061 \mathrm{E}+02 \quad 8.061 \mathrm{E}+02 \quad 1.201 \mathrm{E}-01$ 7. $371 \mathrm{E}+02 \quad 7.371 \mathrm{E}+02 \quad 8.061 \mathrm{E}+02 \quad \mathrm{~B} .061 \mathrm{E}+02 \quad 8.061 \mathrm{E}+02 \quad 1.201 \mathrm{E}-01$ $7.457 \mathrm{E}+02 \quad 7.457 \mathrm{E}+02 \quad 8.147 \mathrm{E}+02 \quad 8.147 \mathrm{E}+02 \quad 8.147 \mathrm{E}+02 \quad 4.151 \mathrm{E}-02$ 7. $339 \mathrm{E}+02 \quad 7.339 \mathrm{E}+02 \quad 7.339 \mathrm{E}+02 \quad 7.343 \mathrm{E}+02 \quad 7.343 \mathrm{E}+02 \quad 8.070 \mathrm{E}+01$ $7.498 \mathrm{E}+02 \quad 7.49 \mathrm{BE}+02 \quad 7.498 \mathrm{E}+02 \quad 7.498 \mathrm{E}+02 \quad 7.498 \mathrm{E}+02 \quad 3.254 \mathrm{E}+00$ $7.328 \mathrm{E}+02 \quad 7.326 \mathrm{E}+02 \quad 7.328 \mathrm{E}+02 \quad 7.333 \mathrm{E}+02 \quad 7.333 \mathrm{E}+02 \quad 2.825 \mathrm{E}+01$ $7.905 \mathrm{E}+02 \quad 7.905 \mathrm{E}+02 \quad 7.905 \mathrm{E}+02 \quad 7.908 \mathrm{E}+02 \quad 7.90 \mathrm{gE}+02 \quad 4.617 \mathrm{E}-01$ $7.334 \mathrm{E}+02 \quad 7.334 \mathrm{E}+02 \quad 7.334 \mathrm{E}+02 \quad 7.334 \mathrm{E}+02 \quad 7.334 \mathrm{E}+02 \quad 1.525 \mathrm{E}+00$ $7.333 \mathrm{E}+02 \quad 7.333 \mathrm{E}+02 \quad 7.333 \mathrm{E}+02 \quad 7.333 \mathrm{E}+02 \quad 7.333 \mathrm{E}+02 \quad 1.777 \mathrm{E}+00$ $7.334 \mathrm{E}+02 \quad 7.334 \mathrm{E}+02 \quad 7.334 \mathrm{E}+02 \quad 7.334 \mathrm{E}+02 \quad 7.334 \mathrm{E}+02 \quad 1.525 \mathrm{E}+00$ $7.330 \mathrm{E}+02 \quad 7.330 \mathrm{E}+02 \quad 7.330 \mathrm{E}+02 \quad 7.330 \mathrm{E}+02 \quad 7,330 \mathrm{E}+02 \quad 2.566 \mathrm{E}+00$ $7.334 \mathrm{E}+02 \quad 7.334 \mathrm{E}+02 \quad 7.334 \mathrm{E}+02 \quad 7.334 \mathrm{E}+02 \quad 7.334 \mathrm{E}+02 \quad 1.525 \mathrm{E}+00$ $7.330 \mathrm{E}+02 \quad 7.330 \mathrm{E}+02 \quad 7.330 \mathrm{E}+02 \quad 7.340 \mathrm{E}+02 \quad 7.340 \mathrm{E}+02 \quad 2.940 \mathrm{E}+00$ $7.351 \mathrm{E}+027.351 \mathrm{E}+02 \quad 7.351 \mathrm{E}+02 \quad 7.354 \mathrm{E}+02 \quad 7.354 \mathrm{E}+02 \quad 7.641 \mathrm{E}-01$ $7.331 \mathrm{E}+02 \quad 7.331 \mathrm{E}+02 \quad 7.331 \mathrm{E}+02 \quad 7.332 \mathrm{E}+02 \quad 7.332 \mathrm{E}+02 \quad 2.273 \mathrm{E}+00$ $8.493 \mathrm{E}+02$ g. $493 \mathrm{E}+02$ g. $493 \mathrm{E}+02$ 8. $494 \mathrm{E}+02$ 白. $494 \mathrm{E}+02$ 5. $277 \mathrm{E}-01$ 8. $270 \mathrm{E}+02 \quad 8.270 \mathrm{E}+02$ g. $270 \mathrm{E}+02 \quad 8.270 \mathrm{E}+02$ 白. $270 \mathrm{E}+02 \quad 5.224 \mathrm{E}-01$ $7.337 \mathrm{E}+027.337 \mathrm{E}+02 \quad 7.337 \mathrm{E}+02 \quad 7.33 \mathrm{E}+02 \quad 7.33 \mathrm{BE}+02 \quad 1.201 \mathrm{E}+00$ $7.328 \mathrm{E}+02 \quad 7.32 \mathrm{EE}+02 \quad 7.328 \mathrm{E}+02 \quad 7.328 \mathrm{E}+02 \quad 7.32 \mathrm{BE}+02 \quad 1.266 \mathrm{E}+01$ $7.352 \mathrm{E}+02 \quad 7.352 \mathrm{E}+02 \quad 7.352 \mathrm{E}+02 \quad 7.357 \mathrm{E}+02 \quad 7.357 \mathrm{E}+02 \quad 7.206 \mathrm{E}-01$ $7.32 \mathrm{BE}+02 \quad 7.328 \mathrm{E}+02 \quad 7.328 \mathrm{E}+02 \quad 7.32 \mathrm{BE}+02 \quad 7.328 \mathrm{E}+02 \quad 5.498 \mathrm{E}+00$ $7.328 \mathrm{E}+02 \quad 7.32$ 政+02 $7.328 \mathrm{E}+02 \quad 7.329 \mathrm{E}+02 \quad 7.329 \mathrm{E}+02 \quad 7.604 \mathrm{E}+00$ $7.328 \mathrm{E}+02 \quad 7.328 \mathrm{E}+02 \quad 7.328 \mathrm{E}+02 \quad 7.328 \mathrm{E}+02 \quad 7.328 \mathrm{E}+02 \quad 1.898 \mathrm{E}+01$ $7.342 \mathrm{E}+02 \quad 7.342 \mathrm{E}+02 \quad 7.342 \mathrm{E}+02 \quad 7.343 \mathrm{E}+02 \quad 7.343 \mathrm{E}+02 \quad 9.609 \mathrm{E}-01$ $7 . \$ 51 \mathrm{E}+02 \quad 7.351 \mathrm{E}+02 \quad 7.351 \mathrm{E}+02 \quad 7.357 \mathrm{E}+02 \quad 7.357 \mathrm{E}+02 \quad 7.641 \mathrm{E}-01$ $7.328 \mathrm{E}+02 \quad 7.328 \mathrm{E}+02 \quad 7.328 \mathrm{E}+02 \quad 7.328 \mathrm{E}+02 \quad 7.328 \mathrm{E}+02 \quad 3.668 \mathrm{E}+01$ $7.329 \mathrm{E}+02 \quad 7.329 \mathrm{E}+02 \quad 7.329 \mathrm{E}+02 \quad 7.329 \mathrm{E}+02 \quad 7.329 \mathrm{E}+02 \quad 2.529 \mathrm{E}+02$ $7.329 \mathrm{E}+02 \quad 7.329 \mathrm{E}+02 \quad 7.328 \mathrm{E}+02 \quad 7.329 \mathrm{E}+02 \quad 7.329 \mathrm{E}+02 \quad 1.813 \mathrm{E}+02$ $7.328 \mathrm{E}+02 \quad 7.328 \mathrm{E}+02 \quad 7.328 \mathrm{E}+02 \quad 7.328 \mathrm{E}+02 \quad 7.328 \mathrm{E}+02 \quad 1.138 \mathrm{E}+02$ $7.328 \mathrm{E}+02 \quad 7.328 \mathrm{E}+02 \quad 7.328 \mathrm{E}+02 \quad 7.328 \mathrm{E}+02 \quad 7.32 \mathrm{gE}+02 \quad 9.708 \mathrm{E}+0 \mathrm{D}$ $7.328 \mathrm{E}+02 \quad 7.328 \mathrm{E}+02 \quad 7.328 \mathrm{E}+02 \quad 7.328 \mathrm{E}+02 \quad 7,328 \mathrm{E}+02 \quad 6.340 \mathrm{E}+00$ $7.328 \mathrm{E}+02 \quad 7.32 \mathrm{BE}+02 \quad 7.328 \mathrm{E}+02 \quad 7.328 \mathrm{E}+02 \quad 7.328 \mathrm{E}+02 \quad 3.246 \mathrm{E}+01$ $7.328 \mathrm{E}+02 \quad 7.328 \mathrm{E}+02 \quad 7.328 \mathrm{E}+02 \quad 7.328 \mathrm{E}+02 \quad 7.328 \mathrm{E}+02 \quad 8.445 \mathrm{E}+00$ 
Chlorometh o-chloraTu

cumene

Dibromochloro

Dichlorodiflo

12ciadichloro

12transDich]

12Dichlprop

Ethylbenz

Nhexane

MethylIso

Propglycol

Styrene

1112 Tetra

1122 Tetra

Trichloflo

123 Frichlopr

Frimethbenz

135Trimeth

acetophenone

ethylchlorid

Methona 1

Metacrylate

cyanide

Dibenz $[a, h]$

24Dinitrotoluene

26Dinitrotoluene

Endasulf an

Avitrobenzenamin

4 Nitrophenol

NNitrosodiphen

$\mathrm{X}_{Y}$ lene

12Dichloro

13Dichloro

14Dichloro

Methylcyc10

$2 \mathrm{Nitrophenol}$

EnoiosulfanI
$7.328 \mathrm{E}+02 \quad 7.328 \mathrm{E}+02 \quad 7.32 \mathrm{gE}+02 \quad 7,328 \mathrm{E}+02 \quad 7.32 \mathrm{BE}+02 \quad 4.637 \mathrm{E}+01$ $7.332 \mathrm{E}+02 \quad 7.333 \mathrm{E}+02 \quad 7.332 \mathrm{E}+02 \quad 7.333 \mathrm{E}+02 \quad 7.333 \mathrm{E}+02 \quad 1.775 \mathrm{E}+00$ $7.334 \mathrm{E}+02 \quad 7.334 \mathrm{E}+02 \quad 7.334 \mathrm{E}+02 \quad 7.335 \mathrm{E}+02 \quad 7.335 \mathrm{E}+02 \quad 1.525 \mathrm{E}+00$ $7.32 \mathrm{gE}+02 \quad 7.328 \mathrm{E}+02 \quad 7.328 \mathrm{E}+02 \quad 7.328 \mathrm{E}+02 \quad 7.328 \mathrm{E}+02 \quad 8.445 \mathrm{E}+00$ $7.328 \mathrm{E}+02 \quad 7.328 \mathrm{E}+02 \quad 7.328 \mathrm{E}+02 \quad 7.328 \mathrm{E}+02 \quad 7.32 \mathrm{BE}+02 \quad 8.445 \mathrm{E}+00$ $7.328 \mathrm{E}+02 \quad 7.32 \mathrm{gE}+02 \quad 7.328 \mathrm{E}+02 \quad 7.32 \mathrm{BE}+02 \quad 7.328 \mathrm{E}+02 \quad 1.266 \mathrm{E}+01$ $7.328 \mathrm{E}+02 \quad 7.328 \mathrm{E}+02 \quad 7.328 \mathrm{E}+02 \quad 7.32 \mathrm{BE}+02 \quad 7.328 \mathrm{E}+02 \quad 8.431 \mathrm{E}+0 \mathrm{I}$ $7.328 \mathrm{E}+027.32 \mathrm{EE}+02 \quad 7.328 \mathrm{E}+02 \quad 7.328 \mathrm{E}+02 \quad 7.328 \mathrm{E}+02 \quad 1.097 \mathrm{E}+01$ $7.330 \mathrm{E}+02 \quad 7.330 \mathrm{E}+02 \quad 7.330 \mathrm{E}+02 \quad 7.330 \mathrm{E}+02 \quad 7.330 \mathrm{E}+02 \quad 2.605 \mathrm{E}+00$ $7.342 \mathrm{E}+02 \quad 7.342 \mathrm{E}+02 \quad 7.342 \mathrm{E}+02 \quad 7.342 \mathrm{E}+02 \quad 7.342 \mathrm{E}+02 \quad 9.609 \mathrm{E}-01$ 7. $32 \mathrm{BE}+02 \quad 7,328 \mathrm{E}+02 \quad 7.328 \mathrm{E}+02 \quad 7.328 \mathrm{E}+02 \quad 7.32 \mathrm{EE}+02 \quad 3.246 \mathrm{E}+01$ $7.334 \mathrm{E}+02 \quad 7.334 \mathrm{E}+02 \quad 7,332 \mathrm{E}+02 \quad 7.334 \mathrm{E}+02 \quad 7.334 \mathrm{E}+02 \quad 1.560 \mathrm{E}+03$ $7.329 \mathrm{E}+02 \quad 7.329 \mathrm{E}+02 \quad 7.329 \mathrm{E}+02 \quad 7.330 \mathrm{E}+02 \quad 7.336 \mathrm{E}+02 \quad 3.358 \mathrm{E}+00$ $7.330 \mathrm{E}+02 \quad 7.330 \mathrm{E}+02 \quad 7.330 \mathrm{E}+02 \quad 7.330 \mathrm{E}+02 \quad \% .330 \mathrm{E}+02 \quad 2.940 \mathrm{E}+00$ $7.328 \mathrm{E}+02 \quad 7.32 \mathrm{BE}+02 \quad 7.328 \mathrm{E}+02 \quad 7.328 \mathrm{E}+02 \quad 7.328 \mathrm{E}+02 \quad 6.340 \mathrm{E}+0 \mathrm{D}$ $7.328 \mathrm{E}+02 \quad 7.328 \mathrm{E}+02 \quad 7.328 \mathrm{E}+02 \quad 7.328 \mathrm{E}+02 \quad 7.328 \mathrm{E}+02 \quad 5.49 \mathrm{BE}+00$ $7.400 \mathrm{E}+02 \quad 7.400 \mathrm{E}+02 \quad 7.400 \mathrm{E}+02 \quad 7.400 \mathrm{E}+02 \quad 7.400 \mathrm{E}+02 \quad 4.926 \mathrm{E}-01$ $7.339 \mathrm{E}+02 \quad 7.339 \mathrm{E}+02 \quad 7.339 \mathrm{E}+02 \quad 7.339 \mathrm{E}+02 \quad 7.339 \mathrm{E}+02 \quad 1.077 \mathrm{E}+00$ $7.333 \mathbf{E}+02 \quad 7.333 \mathbf{E}+02 \quad 7.333 \mathbf{E}+02 \quad 7.333 \mathrm{E}+02 \quad 7.333 \mathrm{E}+02 \quad 1.690 \mathrm{E}+00$ $7.328 \mathrm{E}+1627.328 \mathrm{E}+02 \quad 7.32 \mathrm{gE}+027.328 \mathrm{E}+02 \quad 7.32 \mathrm{BE}+02 \quad 1.645 \mathrm{E}+01$ $7.328 \mathrm{E}+027.32 \mathrm{BE}+02 \quad 7.32 \mathrm{gE}+02 \quad 7.32 \mathrm{gE}+02 \quad 7.32 \mathrm{BE}+02 \quad 2.488 \mathrm{E}+01$ $7.330 \mathrm{E}+02 \quad 7.330 \mathrm{E}+02 \quad 7.329 \mathrm{E}+02 \quad 7.330 \mathrm{E}+02 \quad 7.330 \mathrm{E}+02 \quad 4.637 \mathrm{E}+02$ $7.328 \mathrm{E}+02 \quad 7.328 \mathrm{E}+02 \quad 7.328 \mathrm{E}+02 \quad 7.328 \mathrm{E}+02 \quad 7.32 \mathrm{BE}+02 \quad 2.825 \mathrm{E}+01$ $7.328 \mathrm{E}+02 \quad 7.328 \mathrm{E}+02 \quad 7.570 \mathrm{E}+02 \quad 7.582 \mathrm{E}+02 \quad 7.5 \mathrm{E} 2 \mathrm{E}+02 \quad 3.668 \mathrm{E}+01$ 1. 白白E+03 $1.898 \mathrm{E}+03 \quad 1.941 \mathrm{E}+03 \quad 1.955 \mathrm{E}+03 \quad 1.949 \mathrm{E}+03 \quad 1.255 \mathrm{E}+00$ 7.32 白 $+02 \quad 7.32 \mathrm{gE}+02 \quad 7.770 \mathrm{E}+02 \quad 7.770 \mathrm{E}+02 \quad 7.770 \mathrm{E}+02 \quad 1.097 \mathrm{E}+01$ $7.329 \mathrm{E}+02 \quad 7.32$ 的+02 $7.756 \mathrm{E}+02 \quad 7.756 \mathrm{E}+02 \quad 7.756 \mathrm{E}+02 \quad 1.645 \mathrm{E}+01$ $7.334 \mathrm{E}+02 \quad 7.334 \mathrm{E}+02 \quad 3.661 \mathrm{E}+04 \quad 3.661 \mathrm{E}+04 \quad 3.661 \mathrm{E}+04 \quad 1.444 \mathrm{E}+0 \mathrm{0}$ $7.328 \mathrm{E}+02 \quad 7.328 \mathrm{E}+02 \quad 7.357 \mathrm{E}+07 \quad 7.357 \mathrm{E}+03 \quad 7.357 \mathrm{E}+03 \quad 2.867 \mathrm{E}+01$ $7.32 \mathrm{BE}+02 \quad 7.328 \mathrm{E}+02 \quad 2.872 \mathrm{E}+03 \quad 2.872 \mathrm{E}+03 \quad 2.872 \mathrm{E}+03 \quad 1.266 \mathrm{E}+01$ $7.330 \mathrm{E}+02 \quad 7.330 \mathrm{E}+02 \quad 7.696 \mathrm{E}+02 \quad 7.696 \mathrm{E}+02 \quad 7.696 \mathrm{E}+02 \quad 2.605 \mathrm{E}+00$ $7.332 \mathrm{E}+02 \quad 7.332 \mathrm{E}+02 \quad 1.113 \mathrm{E}+03 \quad 1.113 \mathrm{E}+03 \quad 1.113 \mathrm{E}+03 \quad 1.9 \mathrm{E} \mathrm{E}+00$ $7.332 \mathrm{E}+02 \quad 7.333 \mathrm{E}+02 \quad 1.334 \mathrm{E}+03 \quad 1.334 \mathrm{E}+03 \quad 1.334 \mathrm{E}+03 \quad 1.775 \mathrm{E}+00$ $7.335 \mathrm{E}+02 \quad 7.335 \mathrm{E}+02 \quad 1.424 \mathrm{E}+03 \quad 1.424 \mathrm{E}+03 \quad 1.424 \mathrm{E}+03 \quad 1,362 \mathrm{E}+00$ $7.332 \mathrm{E}+02 \quad 7.332 \mathrm{E}+021.347 \mathrm{E}+03 \quad 1.347 \mathrm{E}+03 \quad 1.347 \mathrm{E}+03 \quad 1.775 \mathrm{E}+00$ $7.329 \mathrm{E}+02 \quad 7.329 \mathrm{E}+02 \quad 1.561 \mathrm{E}+03 \quad 1.561 \mathrm{E}+03 \quad 1.561 \mathrm{E}+03 \quad 3.526 \mathrm{E}+00$

$7.328 \mathrm{E}+02 \quad 7.32 \mathrm{gE}+02 \quad 7.32 \mathrm{gE}+02 \quad 7.32 \mathrm{E}+02 \quad 7.32 \mathrm{BE}+02 \quad 1.519 \mathrm{E}+01$ $\begin{array}{lllll}7.334 \mathrm{E}+02 & 7.334 \mathrm{E}+02 & 7.334 \mathrm{E}+02 & 7.334 \mathrm{E}+02 \quad 7.334 \mathrm{E}+02 \quad 1.444 \mathrm{E}+00\end{array}$ 
Aldehyde

Ketone

Chlorobenzene

vinyl Chloride

o-cresol

m-crespl

p-cresol

140 ichl orobenzen

Hexachloroberzer

Hexachloroethane

Nitrobenzene

246-Trichlorphnl Pyrlaine

24-D

245-TP \&Silvex

Endrin

Heptachlor

Heptachlor epoxd

Lindane

124 trimethylb

1 hexanol

2 hexanone

butylbenzene
$7.400 \mathrm{E}+02 \quad 7.400 \mathrm{E}+02 \quad 7.400 \mathrm{E}+02 \quad 7,401 \mathrm{E}+02 \quad 7.401 \mathrm{E}+02 \quad 4.928 \mathrm{~B}-01$

$7.400 \mathrm{E}+02 \quad 7.400 \mathrm{E}+02 \quad 7.400 \mathrm{E}+02 \quad 7.401 \mathrm{E}+02 \quad 7.401 \mathrm{E}+02 \quad 4.928 \mathrm{E}-01$

$7.329 \mathrm{E}+02 \quad 7.329 \mathrm{E}+02 \quad 7.329 \mathrm{E}+02 \quad 7.329 \mathrm{E}+02 \quad 7.329 \mathrm{E}+02 \quad 3.820 \mathrm{E}+00$

$7.328 \mathrm{E}+02 \quad 7.328 \mathrm{E}+02 \quad 7.32 \mathrm{BE}+02 \quad 7.32 \mathrm{BE}+02 \quad 7.328 \mathrm{E}+02 \quad 2.488 \mathrm{E}+01$

$7.328 \mathrm{E}+02 \quad 7.328 \mathrm{E}+02 \quad 7.328 \mathrm{E}+02 \quad 7.32 \mathrm{BE}+02 \quad 7.328 \mathrm{E}+02 \quad 1.266 \mathrm{E}+01$

$7.328 \mathrm{E}+02 \quad 7.328 \mathrm{E}+02 \quad 7,32 \mathrm{BE}+02 \quad 7.328 \mathrm{E}+02 \quad 7.328 \mathrm{E}+02 \quad 1.097 \mathrm{E}+01$

$7.32 \mathrm{BE}+02 \quad 7.32 \mathrm{BE}+02 \quad 7.32 \mathrm{BE}+02 \quad 7.328 \mathrm{E}+02 \quad 7.328 \mathrm{E}+02 \quad 1.266 \mathrm{E}+01$

$7.332 \mathrm{E}+02 \quad 7.332 \mathrm{E}+02 \quad 7.332 \mathrm{E}+02 \quad 7.333 \mathrm{E}+02 \quad 7.333 \mathrm{E}+02 \quad 1.775 \mathrm{E}+00$

$7.695 \mathrm{E}+02 \quad 7.695 \mathrm{E}+02 \quad 7.695 \mathrm{E}+02 \quad 7.696 \mathrm{E}+02 \quad 7.696 \mathrm{E}+02 \quad 4.244 \mathrm{E}-01$

$\begin{array}{llllll}7.342 \mathrm{E}+02 & 7.342 \mathrm{E}+02 & 7.342 \mathrm{E}+02 & 7.343 \mathrm{E}+02 & 7.343 \mathrm{E}+02 & 9.609 \mathrm{E}-01\end{array}$

$7.328 \mathrm{E}+02 \quad 7.328 \mathrm{E}+02 \quad 7.32 \mathrm{E}+02 \quad 7.32 \mathrm{E}+02 \quad 7.328 \mathrm{E}+02 \quad 1.434 \mathrm{E}+01$

$7.337 \mathrm{E}+02 \quad 7.337 \mathrm{E}+02 \quad 7.337 \mathrm{E}+02 \quad 7.337 \mathrm{E}+02 \quad 7.337 \mathrm{E}+02 \quad 1.201 \mathrm{E}+00$

$7.328 \mathrm{E}+02 \quad 7.328 \mathrm{E}+02 \quad 7.328 \mathrm{E}+02 \quad 7.32 \mathrm{gE}+02 \quad 7.32 \mathrm{BE}+02 \quad 2,825 \mathrm{E}+01$

$7.328 \mathrm{~B}+02 \quad 7.328 \mathrm{E}+02 \quad 7.32 \mathrm{gE}+02 \quad 7.328 \mathrm{E}+02 \quad 7.328 \mathrm{E}+02 \quad 5.498 \mathrm{E}+00$

$7.342 \mathrm{E}+02 \quad 7.342 \mathrm{E}+02 \quad 7.342 \mathrm{E}+02 \quad 7.342 \mathrm{E}+02 \quad 7.342 \mathrm{E}+02 \quad 9.609 \mathrm{E}-01$

$7.400 \mathrm{E}+02 \quad 7.400 \mathrm{E}+02 \quad 7.400 \mathrm{E}+02 \quad 7.401 \mathrm{E}+02 \quad 7,401 \mathrm{E}+02 \quad 4.928 \mathrm{E}-01$

$7.365 \mathrm{E}+027.365 \mathrm{E}+02 \quad 7.365 \mathrm{E}+02 \quad 7.366 \mathrm{E}+02 \quad 7.366 \mathrm{E}+02 \quad 6.144 \mathrm{E}-01$

$7.7 \mathrm{g9E}+02 \quad 7.789 \mathrm{E}+02 \quad 7.789 \mathrm{E}+02 \quad 7.7 \mathrm{BgE}+02 \quad 7.769 \mathrm{E}+02 \quad 4.365 \mathrm{E}-01$

$7.337 \mathrm{E}+02 \quad 7.337 \mathrm{E}+02 \quad 7.337 \mathrm{E}+02 \quad 7.33 \mathrm{EE}+02 \quad 7.338 \mathrm{E}+02 \quad 1.201 \mathrm{E}+00$

$7.339 \mathrm{E}+02 \quad 7.339 \mathrm{E}+02 \quad 7.339 \mathrm{E}+02 \quad 7.339 \mathrm{E}+02 \quad 7.339 \mathrm{E}+02 \quad 1.077 \mathrm{E}+00$

7. $32 \mathrm{gE}+02 \quad 7.328 \mathrm{E}+02 \quad 7.32 \mathrm{BE}+02 \quad 7.32 \mathrm{BE}+02 \quad 7.328 \mathrm{E}+02 \quad 2.488 \mathrm{E}+01$

7. $32 \mathrm{gE}+02 \quad 7.328 \mathrm{E}+02 \quad 7.32 \mathrm{BE}+02 \quad 7.326 \mathrm{E}+02 \quad 7.328 \mathrm{E}+02 \quad 2.488 \mathrm{E}+01$

$7.334 \mathrm{E}+02 \quad 7.334 \mathrm{E}+02 \quad 7.334 \mathrm{E}+02 \quad 7.334 \mathrm{E}+02 \quad 7.334 \mathrm{E}+02 \quad 1.525 \mathrm{E}+00$ 


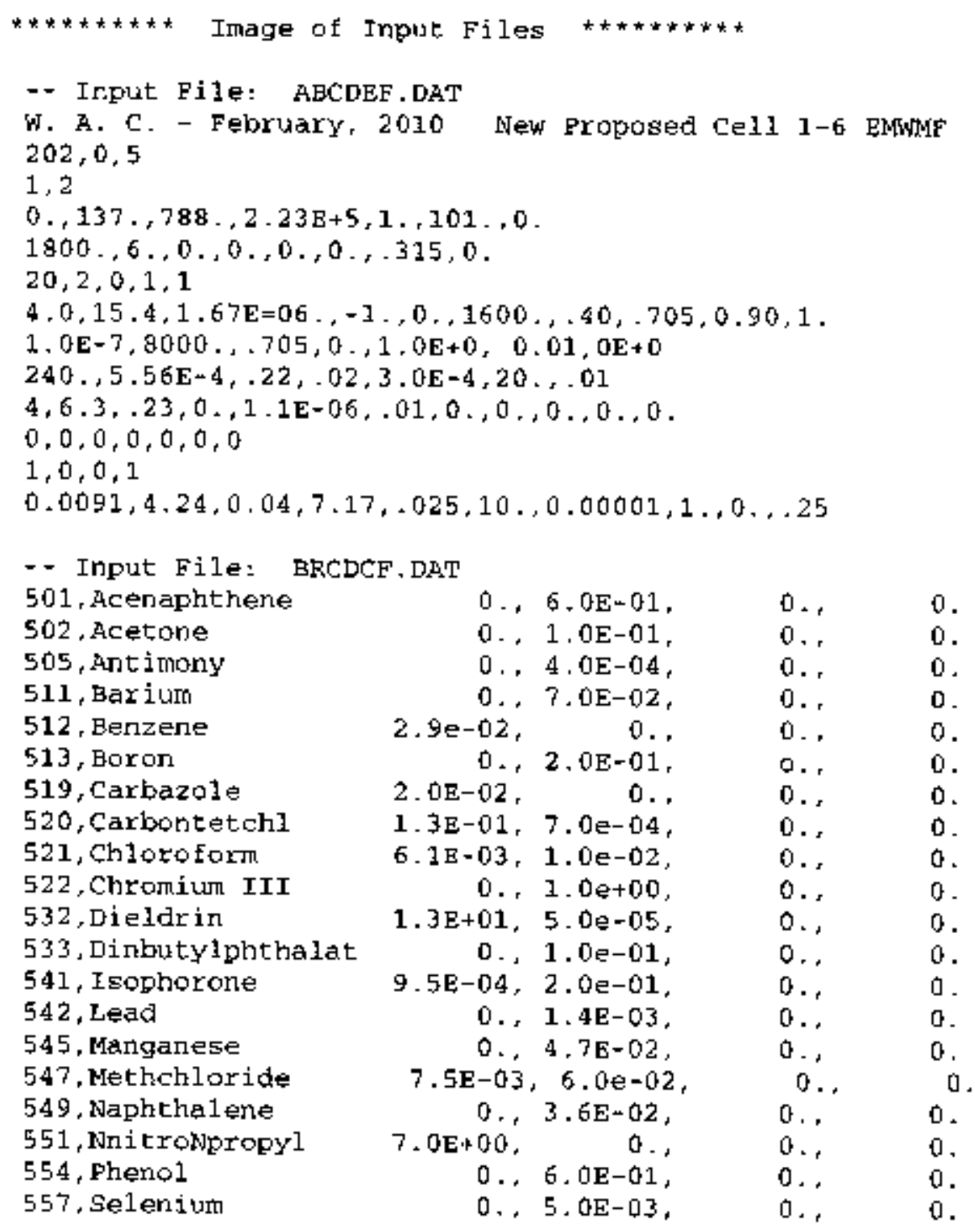


560, Strontium

561, Tetrachloroethen

563, Tin

564, Toluene

565, Trichloroethene

567, U-23

$568, U-234$

$569, \mathrm{U}-235$

$570, U-236$

$571, \mathrm{U}-238$

572 , Varadium

592, 124 Trich1orb

593, Molybdenum

596 , benzidine

597, Chlordane

619. Methyl PropylB

620,124 Trd MethylB

622 , WethylethylB

624, 12DiMethylB

625, PropylB

626, Aldrin

62日, Aroclor 1221

629, Aroclor 1232

$630, \mathrm{DDD}$

$631, \mathrm{DDE}$

633,Alpha-BHC

634 , Beta-BHC

635. Del 다-日HC

636. Acenaphthylene

637, Benzoic Acid

638, Dibenzofurar

639, Diethylphth

640,24-Dimethylphe

641, Dimethylphth

642, 2Methylnaptha

643, 2346Tetrachlor

644, Benzyl Alcohol

645, Acentonitrile
0., 6.0e-01,

5. 2E-02, 1, 0e-02,

0.. 6.0E-01.

0., 2,0e-01.

1. 1E-02,

0 . .

0., 3,0E-03.

D., 3.0E-03.

0, 3.0E-03,

$0 ., 3.02-03$,

$0 ., 3.0 \mathrm{E}-03$,

$0 ., 7.0 \mathrm{E}-03$.

$0.00 \mathrm{e}+00,5.0 \mathrm{e}-02$ s

$0.00 \mathrm{e}+0,5.0 \mathrm{E}-03$,

$2.3 \mathrm{E}+02,3.0 \mathrm{e}-03$,

$3.5 \mathrm{~B}-01,5.0 \mathrm{E}-04$,

$0 ., 3.7$ e 02 .

o., 5.0e-02,

$0.3 .7 e-02$.

$0.2 .00+00$

0., 3.7e-02,

$1.7 \mathrm{E}+01,3.0 e-05$

4. $0 \mathrm{E}-01$.

4. 0E-01.

2. 4E-01.

3. $4 \mathrm{E}-01$.

6. $3 \mathrm{E}+00$,

1. $\dot{\theta} \mathrm{E}+0 \mathrm{D}$,

1. $\mathrm{BE}+00$,

0.6.

(1. 4 . De+00.

$0.4,0$-0. 3 .

0, 8.0e-01,

$0 ., 2.0 \mathrm{e}-02$.

$0 ., 1.0 E+01$.

$0.4 .0 \mathrm{e}-03$.

$0 ., 3.0 \mathrm{e}-02$,

$0 ., 3.0$ e-01.

0. 6.0e-03

$0 ., 0$.

$0 ., 0$.

$0 . .0$

D.. 0

$0, x \quad 0$.

D.,

$0 . .20$

$0 . x \quad 0$

0.50

$0 ., 0$

0.00.

$0 ., 0$

o., 0 .

Q.. 0 .

$0 . .0$.

0.10

0.

0.

0.

$0 ., 0$.

$0 ., 0$

$0 \ldots$ D

ㅁ.. 0 ,

D., 0 .

$0,+0$.

D., 0 .

O. 10

$0 . .0$.

D., 0

D. 0 .

o. $\quad 0$

o.. 0 .

$0 ., 0$.

$0 ., 0$

o.. 0 .

o.. 0 . 
646. Acrolien

647, Acylonitrle

648, Bromodichloro

649, Bromoform

650, Bromameth

651, CarbonDis

652, Chlorometh

653,0 -chlorotw

654 , Cumene

655, Dibromochloro

656, Dichlorodiflo

6.57, 12cisDichloro

658,12 transDichl

659, 12 bichlprop

660, Ethylbenz

661 , Nhexane

662, MethylI so

664, Propolycol

665, Styrene

666, 1112Tetra

667, 1122 Tetra

668, Trichloflo

669.123 Trichlopr

670. Trimethbenz

$671,135 \mathrm{Tr}$ imeth

673 , acecophenone

674, ethylchlorid

676 . Methonal

677, Minetacrylabe

679, Cyanide

680, Dibenz $\left[a_{s} h\right]$

681,24 Dinitrotoluene

685. 4Nitrobenzenamic 2.1E-02, 6.0e-03,

686. $4 \mathrm{Ni}$ trophenol

687. NNi trosodiphen

68 , xylene
$6.8 e-01,1.0 e-03$,
$5.45-01,5.0 \mathrm{e}-04$
6. $2 \mathrm{E}-02,2$, $0 \mathrm{E}-02$
7.9E-03, 2.0E-02,
ㅇ., 1.4E-03,
0., $1,0 \mathbf{E}-01$

1. $3 \mathrm{E}-02$

$$
0 ., 2,0 \mathrm{E}-02 \text {, }
$$

$0,1,0 \mathrm{E}-01$

B , 4E-02, 2, 0E-02,

$0,2.0 \mathrm{e}-01$

0 . 1. 0e-02,

D., 2, 0E-02,

6.8E-02, 0.

$0 ., 6.0$ - -02 ,

$0 .$, B. 0 e-02,

$0.5 .0 e-01$

$0 ., 2.0 \mathrm{e}-01$,

$2.6 \mathrm{E}-02,3.0 \mathrm{e}-02$,

$2,0 \mathbf{E}-01,6.0 \mathrm{e}-02$,

$0.3 .0 \mathbf{E}-01$,

7. $0 \mathrm{E}+00,6.0 \mathrm{e}-03$

$0 ., 5.0 \mathrm{e}-02$.

0.. 5.0e-02,

$0 ., 1,0 e-01$,

2.9E-03, 4.0E-01,

0. 5, 5e-0I,

$0 ., 1,40 e+00$.

$0,2,0 e-02$,

3e+00, $0 .$,

$2.1 \mathrm{E}-02,3.0 \mathrm{e}-03$,
$0 ., 6.2 \mathrm{e}-02$,

4.9e-03, 2.0e-02,

0.. 2.0e-01,

0.20.

$0 .$.

$0 ., \quad 0$.

$0 ., \quad 0$

$0 .+0$

$0 . .0$.

$0,+0$.

$0 ., \quad 0$.

$0, r \quad 0$.

$0 . .0$.

o.. 0 .

o. 0 .

O.. 0.

$0 . . \quad 0$.

o. 0 .

o. 0 .

0.00.

$0 ., 0$.

$0 . .00$.

$0 . .00$.

$0 ., 0$,

$0 ., 0$.

$0, r \quad 0$.

$0 . r \quad 0$.

$0 . .0$.

$0 . .0$.

$0 ., \quad 0$.

0 .

$0 ., 0$.

D. 0 .

D. 0 .

. 0 .

$0 . .0$.

0.10

o.. 아 


\begin{tabular}{|c|c|c|}
\hline 689,12 Dichloro & $0,9.0 \mathrm{e}-02$, & 0. \\
\hline $690,13 \mathrm{Dich}$ loro & $0,8.9 \mathrm{e}-02$ & 6. \\
\hline 691,14Dichloro & $2.4 e-02,2.3 e-01$, & $0 \ldots$ \\
\hline 692, Methyleyclo & $0.6 .0 \mathrm{e}-02$ & 0. \\
\hline $696,2 \mathrm{Nitrophenol}$ & $0 ., 6.20 \mathrm{E}-02$ & 0. \\
\hline 69⿱㇒日, EndosulfanII & $0.6 .00-03$ & $0 \ldots$ \\
\hline 699, Aldehyde & $0.3 .3 \mathrm{E}-04$, & 0. \\
\hline 700, Ketone & $0 ., 3-0 e-04$ & 0. \\
\hline 701, chlorobenzene & 0., 2,0 e- 02 & 0, \\
\hline 702, vinyl Chloride & $1,4 e+00,3.0 e-03$. & 0. \\
\hline 703, o-cresol & $0.5 .0 e-02$ & 0. \\
\hline 704, Ji-cresol & $0.5,0 \mathrm{e}-02$. & 0. \\
\hline $705, p-c r e s o l$ & $0,5,0 \mathrm{e}-03$ & 0. \\
\hline 706, 140i chl orobenzen & $2,4 e-02,2.3 \mathrm{E}-01$, & $0 .$. \\
\hline 707, Hexachloroberzen & $1.6 e+00,8.0 e-04$. & 0. \\
\hline 709, Hexachloroetharie & 1. $4 \mathrm{e}-02,1,0 \mathrm{e}-03$ & 0 \\
\hline 710, Nitrobenzere & $5.0 \mathrm{e}-04$ & 0. \\
\hline 711, 246-Trichlorphnl & 1. 1e-02, & 0. \\
\hline 712, Pyridine & $0 . .1 .0 e-03$ & 0. \\
\hline $713,24=D$ & $0 ., 1.0 \mathrm{e}=02$, & o. \\
\hline $714,245=\mathrm{TP}$ (Silvex) & 0., $8.0 e-03$. & 0. \\
\hline 716, Endrin & $3.0 \mathrm{e}-04$ & 0. \\
\hline 717, Heptachlor & $5.0 e-04$. & 0. \\
\hline 718, Heptachlor epoxd & $9.1 e+0,1.3 e-0.5$. & 0. \\
\hline 719, Lindane & $1,3 e+0,3,0 e-04$, & 0. \\
\hline 723,124 trimethylb & $0 ., 5.00-02$ & 0, \\
\hline 724,1 hexanol & $0,4,0 \mathrm{E}-02$, & 0. \\
\hline 725 , 2hexanone & $0,4.0 \mathrm{E}-02$ & 0. \\
\hline 726, butylbenzene & $0 ., 3 . \mathrm{BE}-02$, & 0.1 \\
\hline
\end{tabular}

-- Input File: INVNTRY. DAT 501, 1.00e+10,1.67E+06, $502,1.00 e+10,1.67 \mathrm{E}+06$, $505,1.00 e+10,1.67 \mathrm{E}+06$, $511,1,00 \mathrm{E}+10,1,67 \mathrm{E}+06$ $512,1,00 \mathrm{f}+10,1.67 \mathrm{E}+06$ $513,1,00 \mathrm{E}+10,1,67 \mathrm{E}+06$,

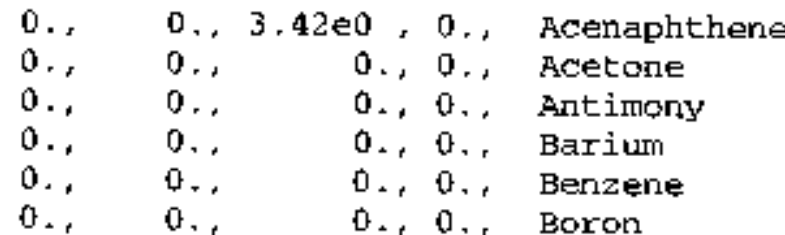


$519,1.000+10,1.67 E+06$ $520,1.000+10,1.67 \mathrm{E}+06$, $521,1.00 \mathrm{e}+10,1.67 \mathrm{E}+06$, $522,1.00 \mathrm{E}+10,1.67 \mathrm{E}+06$, $532,1.00 E+10,1.67 E+06$, $533,1.00 \mathrm{e}+10,1.67 \mathrm{E}+06$. $541,1.00 \mathrm{e}+10,1.67 \mathrm{E}+06$, $542,1.00 \mathrm{E}+10,1,67 \mathrm{E}+06$, $545,1,00 \mathrm{E}+10,1,67 \mathrm{E}+06$, 547, 1.00e+10,1.67E+06, $549,1.00 \mathrm{E}+10,1.67 \mathrm{E}+06$, $551,1.00 \mathrm{E}+10,1.67 \mathrm{E}+06$. $554,1.00 \mathrm{E}+10,1.67 \mathrm{E}+06$. $557,1.00 \mathrm{E}+10,1.67 \mathrm{E}+06$. $560,1.00 \mathrm{E}+10,1.67 \mathrm{E}+06$, $561,1.00 \mathrm{e}+10,1.67 \mathrm{E}+06$, $563,1.00 \mathrm{E}+10,1.67 \mathrm{E}+06$, $564,1.00 \mathrm{e}+10,1.67 \mathrm{E}+06$, $565,1.00 \mathrm{e}+10,1.67 \mathrm{E}+06$, $567,1.59 \mathrm{E}+05,1.67 \mathrm{E}+06$, $568,2.44 \mathrm{E}+05,1.67 \mathrm{E}+06$, $569,7.04 \mathrm{E}+08,1.67 \mathrm{E}+06$, $570,2.34 E+07,1.67 E+06$, $571,4.47 \mathrm{E}+09,1.67 \mathrm{E}+06$ 。 $572,1,00 \mathrm{E}+10,1.67 \mathrm{E}+06$, $592,1,00 \mathrm{E}+10,1,67 \mathrm{E}+06$, $593,1.00 E+10,1+67 \mathrm{E}+06$, $592,1,00 \mathrm{E}+10,1,67 \mathrm{E}+06$, $598,1.00 \mathrm{E}+10,1.67 \mathrm{E}+06$, $619,1,00 \mathrm{E}+10,1,67 \mathrm{E}+06$, $620,1,00 \mathrm{E}+10,1.67 \mathrm{E}+06$, $622,1.00 \mathrm{E}+10,1.67 \mathrm{E}+06$, $624,1.00 \mathrm{E}+10,1.67 \mathrm{E}+06$. $625,1.00 \mathrm{E}+10,1.67 \mathrm{E}+06$, $626,1.00 \mathrm{E}+10,1.67 \mathrm{E}+06$, $628,1.00 \mathrm{E}+10,1.67 \mathrm{E}+06$. $629,1.00 \mathrm{E}+10,1.67 \mathrm{E}+06$, $630,1.00 \mathrm{E}+10,1.67 \mathrm{E}+06$

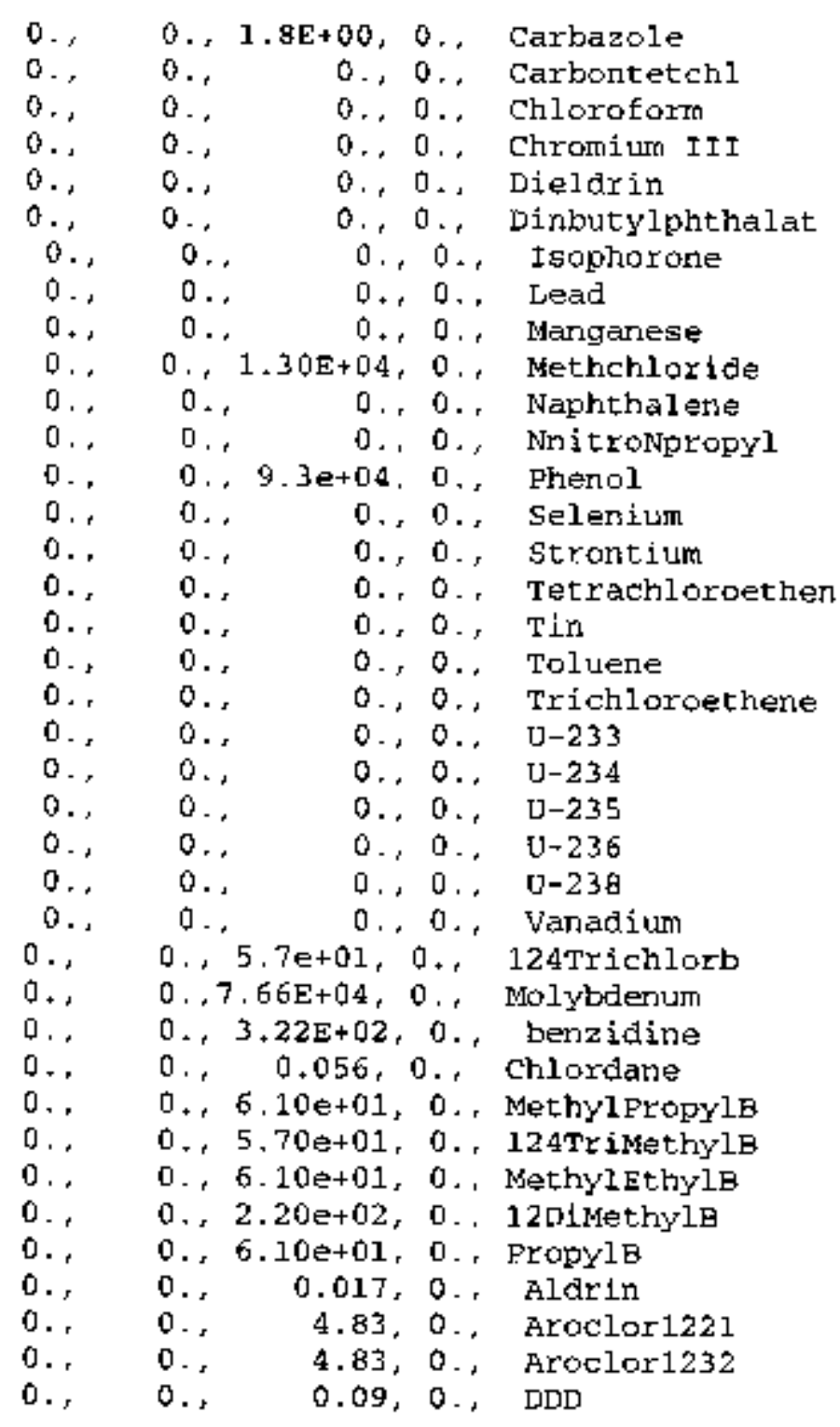


$631,1.00 \mathrm{E}+10,1.67 \mathrm{E}+06$, $633,1.00 \mathrm{E}+10,1,67 \mathrm{E}+06$, $634,1.00 \mathrm{E}+10,1.67 \mathrm{E}+06$, $635,1.00 \mathrm{E}+10,1.67 \mathrm{E}+06$ $636,1.00 E+10,1.67 E+06$, $637,1.00 \mathrm{E}+10,1.67 \mathrm{E}+06$, $63 \mathrm{~B}, 1,00 \mathrm{E}+10,1,67 \mathrm{E}+06$, 639, $1.00 \mathrm{E}+10,1,67 \mathrm{E}+06$, $640,1.00 \mathrm{E}+10,1.67 \mathrm{E}+06$, $641,1-00 \mathrm{E}+10,1.67 \mathrm{E}+06$, $642,1.00 \mathrm{E}+10,1,67 \mathrm{E}+06$, $643,1.00 \mathrm{E}+10,1-67 \mathrm{E}+06$, $644,1.00 \mathrm{E}+10,1.67 \mathrm{E}+06$. $645,1.00 \mathrm{E}+10,1.67 \mathrm{E}+06$. 646. 1.00E+10,1.67E+06. $647,1.00 \mathrm{E}+10,1,67 \mathrm{E}+06$, $646,1.00 \mathrm{E}+10,1.67 \mathrm{E}+06$, $649,1.00 \mathrm{E}+10,1.67 \mathrm{E}+06$, $650,1.00 \mathrm{E}+10,1.67 \mathrm{E}+06$ $651,1.00 \mathrm{E}+10,1.67 \mathrm{E}+06$, $652,1.00 \mathrm{E}+10,1.67 \mathrm{~B}+06$, 653. 1.00E+10,1.67E+06, $654,1.00 \mathrm{E}+10.1 .67 \mathrm{E}+06$, $655,1.00 \mathrm{E}+10.1 .67 \mathrm{E}+06$, $656,1.00 \mathrm{E}+10.1 .67 \mathrm{E}+06$. $657,1.00 \mathrm{E}+10,1.67 \mathrm{E}+06$ $658,1.00 \mathrm{E}+10,1.67 \mathrm{E}+06$, $659,1.00 \mathrm{E}+10,1.67 \mathrm{E}+06$, $660,1,00 \mathrm{E}+10,1,67 \mathrm{E}+06$, 661, 1.00E+10,1.67E+06, $662,1.00 E+10,1.67 E+06$, $664,1.00 \mathrm{E}+10,1.67 \mathrm{E}+06$. $665,1.00 \mathrm{E}+10,1,67 \mathrm{E}+06$, $666,1.00 \mathrm{E}+10,1.67 \mathrm{E}+06$, $667,1.00 \mathrm{E}+10,1.67 \mathrm{E}+06$, $668,1.00 \mathrm{E}+10,1.67 \mathrm{E}+06$, $669,1.00 \mathrm{E}+10,1.67 \mathrm{E}+06$, 670. $1,00 \mathrm{E}+10,1.67 \mathrm{E}+06$,

\begin{tabular}{|c|c|c|c|c|}
\hline o.. & $0 .$, & 0.04, & 0. & DDE \\
\hline 0. & 0. & 8. & $0 .$. & A1pha-BHC \\
\hline o., & 0. & 8. & 0. & Beta-BHC \\
\hline 0. & 0. & 8. & 0. & Delta-BHC \\
\hline 0. & o. & $1.610+01$ & $0 \ldots$ & Acenaphthylene \\
\hline 0. & 0. & $3.400+03$ & $0 \ldots$ & Benzolic Acid \\
\hline $0 .$. & o. & $3.1 e+00$ & $0 \ldots$ & Dibenzofuran \\
\hline $0 .$. & $0 .$. & $1.08 \mathrm{e}+03$. & $0 .$. & Diethylphth \\
\hline $0 .$, & $0 \ldots$ & $7.87 e+03$. & $0 .$. & 24-Dimethylphe \\
\hline 0. & $0 .$. & $4.0 e+03$ & 0. & Dimethylphth \\
\hline 0. & $0 .$. & $2.46 e+01$ r & $0 .$. & 2Methylnaptha \\
\hline 0. & 0. & $2.3 e+01$ & 0. & 2306 Tetrachlor \\
\hline O., & 0. & $4.29 e+04$ & 0. & Benzyl Alcohol \\
\hline $0 .$. & $0 \ldots$ & $1.00 \mathrm{E}+06$. & 0. & Acentonitrile \\
\hline $0 .$. & $0 .$. & $.12 \mathrm{E}+05$ & 0. & Acrolien \\
\hline 0. & $0 .$. & $7.45 \mathrm{E}+04 \mathrm{~s}$ & $0 .$. & Acylonitrle \\
\hline 0. & $0 .$. & $3.03 \mathrm{E}+03$ & $0 .$. & Bromodichloro \\
\hline $0 .$. & 0. & $.10 \mathrm{E}+03$ & $0 .$, & Eromoform \\
\hline 0. & 0. & $.52 E+04$ & 0. , & Bromometh \\
\hline 0. & 0. & $1.18 \mathrm{E}+03$ & $0 .$, & Carbondis \\
\hline 0. & 0. & $5.32 \mathrm{E}+03$ & 0. & Chlorometh \\
\hline 0. & $0, s$ & 3. $74 \mathrm{E}+02$ & o.. & o-Chlorotu \\
\hline 0, & $0 .$. & $6.13 \mathrm{E}+01$ & $0 .$. & Cumene \\
\hline $0 .$. & $0 .$, & $2.70 \mathrm{E}+03$ & 0. & Dibromochloro \\
\hline $0 .$, & $0 .$, & $2.80 \mathrm{E}+02$ & 0. & Dichlorodiflo \\
\hline 0. & $0 .$. & $3.50 \mathrm{E}+03$ & a.. & 12cisDichloro \\
\hline 0. & $0 .$. & $3.50 \mathrm{E}+03$ & 0. & 12transDichl \\
\hline 0. & $0 .$. & $2.80 E+03$. & $0 .$. & 12Dich1prop \\
\hline 0. & 0. & $1.69 E+02$ & 0. & Ethylbenz \\
\hline $0 .$. & a., & $9.50 \mathrm{E}+00$ & 0. & Nhexane \\
\hline $0 .$. & $0 .$. & $1.90 E+04$ & a. & Methyliso \\
\hline a., & $0 \ldots$ & $1.00 E+06$ & a. & PropGlycol \\
\hline O.. & $0 .$. & $3.10 \mathrm{~b}+02$. & 0. & Styrene \\
\hline O.. & $0 .$, & $1.07 E+03$ r & $0 .$. & ll12Tetra \\
\hline $0 .$. & $0 .$, & $2.87 E+03$. & 0. & l122Tetra \\
\hline D.. & a., & $1.10 \mathrm{E}+03$. & o. & Trichloflo \\
\hline 0. & $0 .$. & 1. $75 E+03$. & $0 .$. & 123Trichlopr \\
\hline $0 .$. & $0,$. & $5.70 \mathrm{E}+01 \mathrm{x}$ & & Trimethbenz \\
\hline
\end{tabular}


$671,1.00 \mathrm{E}+10,1,67 \mathrm{E}+06$. 673, 1.00E+10,1.67E+06, $674,1.00 \mathrm{E}+10,1.67 \mathrm{E}+06$, 676, $1.00 \mathrm{E}+10,1.67 \mathrm{E}+06$, $677,1.00 \mathrm{E}+10,1.67 \mathrm{E}+06$, $679,1.00 \mathrm{E}+10,1.67 \mathrm{E}+0.6$. $680,1,00 \mathrm{E}+10,1,67 \mathrm{E}+06$. 681, 1.00E+10,1,67E+06. $682,1.00 \mathrm{E}+10,1,67 \mathrm{E}+06$, $684,1,00 \mathrm{E}+10,1,67 \mathrm{E}+06$, $685,1.00 \mathrm{E}+10,1.67 \mathrm{E}+06$ ， $686,1.00 \mathrm{E}+10,1.67 \mathrm{E}+06$. $687,1.00 \mathrm{E}+10,1.67 \mathrm{E}+06$, $688,1.00 \mathrm{E}+10,1.67 \mathrm{E}+06$, $689,1.00 \mathrm{E}+10,1.67 \mathrm{E}+06$, $690,1.00 \mathrm{E}+10,1.67 \mathrm{E}+06$, $691,1.00 \mathrm{E}+10,1.67 \mathrm{E}+06$, $692,1,00 \mathrm{E}+10,1.67 \mathrm{E}+06$, $696,1.00 \mathrm{E}+10,1.67 \mathrm{E}+06$, $69 \%, 1.00 \mathrm{E}+10,1.67 \mathrm{E}+06$, $699,1.00 \mathrm{E}+10,1.67 \mathrm{E}+06$, $700,1.00 \mathrm{E}+10,1.67 \mathrm{E}+06$. $701,1.00 \mathrm{E}+10,1.67 \mathrm{E}+06$, $702,1.00 \mathrm{E}+10,1.67 \mathrm{E}+06$. 703, 1.00E+10,1.67E+06, $704,1.00 \mathrm{E}+10,1.67 \mathrm{E}+06$ $705,1.00 \mathrm{E}+10,1.67 \mathrm{E}+06$. $706,1.00 E+10,1.67 E+06$, 707. 1.00E+10, 1.67E+06, $709,1.00 \mathrm{E}+10,1.67 \mathrm{E}+06$, $710,1.00 \mathrm{E}+10.1 .67 \mathrm{E}+06$, $711,1.00 \mathrm{E}+10,1.67 \mathrm{E}+06$, $712,1.00 \mathrm{E}+10,1.67 \mathrm{E}+06$. $713,1,00 \mathrm{E}+10,1,67 \mathrm{E}+06$. $714,1.00 \mathrm{E}+10,1+67 \mathrm{E}+06$, $716,1.00 \mathrm{E}+10,1.67 \mathrm{E}+06$. $717,1.00 \mathrm{E}+10,1,67 \mathrm{E}+06$. $718,1.00 \mathrm{E}+10,1.67 \mathrm{E}+06$,

\begin{tabular}{|c|c|c|c|c|}
\hline 0. . & $0 .$. & $4.82 \mathrm{E}+01$ & $0 .$. & 135Trimeth \\
\hline a. , & $0 .$. & $6.13 \mathrm{E}+03$ & 0. & acetophenone \\
\hline $0 .$. & o.. & $6.70 \mathrm{E}+03$ & 0. & Ethylchlorid \\
\hline .. & 0. & $1.00 \mathrm{E}+06$ & $0 \ldots$ & Methonal \\
\hline o., & o.., & $1.500+04$ & 0. & PMetacrylate \\
\hline $0 .$. & $0 .$. & $1.00 e+06$ & 0. & Cyanide \\
\hline 0. & 0. & $1+03 \mathrm{e}-03$ & 0. & Dibenz $[a, h]$ \\
\hline $0 .$. & $0 .$. & $2.70 \mathrm{e}+02$ & 0. & 24Dinitrotoluene \\
\hline $0 .$. & $0 .$. & $3.52 \mathrm{e}+02$ & 0. & 26Dinitrotoluene \\
\hline a. . & $0 .$, & $4.50 e-01$, & 0. & Endosulfan \\
\hline $0 .$. & $0 .$, & $1.07 e-05$ & 0. & $4 \mathrm{Nitrobenzenamin}$ \\
\hline $0 .$. & O.., & $1.16 e+04$ & 0. & 4Nitrophenol \\
\hline 0. & 0.. & $3.50 \mathrm{e}+01$. & 0. & WNitrosodiphen \\
\hline $0 .$. & $0 .$. & $1.06 \mathrm{E}+02$. & 0, & XYlene \\
\hline Q. . & $0 .$, & $8.00 \mathrm{E}+01$ & 0. & 12Dichloro \\
\hline $0+$. & $0 .$, & $1.25 \mathrm{E}+02$ & 0. & 13Dichloro \\
\hline 0, & 0. & B. $13 \mathrm{E}+01$, & 0. & 14Dichloro \\
\hline $0 .$. & 0. & $1.40 E+01$ & $0 .$. & Methy1 byclo \\
\hline $0 .$. & 0. & $2.5 \mathrm{E}+03$ & 0. & 2Nitrophenol \\
\hline 0. & 0. & 0.45 & o. & EndosufanII \\
\hline 0. & 0. & 0.25 & 0. & Aldehyde \\
\hline 0. & D. & 0.25 & 0. & Ketone \\
\hline 0. & 0. & $4.98=+02$ & $0 .$. & Chlorobenzene \\
\hline 0. & 0. & 8.80403 & 0. & Vinyl Chloride \\
\hline $0 .$, & 0. & $2.59 e+04$ & a... & o-cresol \\
\hline $0 .$. & 0. & $2.27 e+04$ & $0 .$. & m-cresol \\
\hline $0 .$, & 0. & $2.15 e+04$ & o.. & p-cresol \\
\hline o., & 0. & $8.13 \mathrm{e}+01$ & $a_{+}$, & 14Dichlorobenzen \\
\hline o., & 0. & $6.2 \mathrm{e}-03$ & a., & Hexachlorobenzen \\
\hline a.. & 0. & $5.0 \mathrm{e}+01$. & $0 .$. & Hexachloroethane \\
\hline 0. & o., & $2.09 e+03$. & $0 .$. & Nitrobenzere \\
\hline $0 .$. & a., & 8. 0 ee+02. & 0. & 246-Trichlorophni \\
\hline $0 .$. & $0+$. & $1.0 e+06$ & 0. & Eyridine \\
\hline 0. & Q... & $6.82 \mathrm{e}+02$, & $0 .$. & $24-D$ \\
\hline a., & 0. & $2.0 \mathrm{e}+02$ & 0. & 245-TP (Silvex) \\
\hline a. & 0. & $2.5 \mathrm{e}-01$ & 0 & Endrin \\
\hline a. & 0. & $1.8 \mathrm{e}-01$ & 0, & Heptachlor \\
\hline 0. & 0 . & $2.0 e-01$ & 0. & Heptachlor epoxa \\
\hline
\end{tabular}


$719,1,00 \mathrm{E}+10,1,67 \mathrm{E}+06$, $723,1,00 \mathrm{E}+10,1,67 \mathrm{E}+06$. 724, 1.00E+10, 1,67E+06. 725, 1.00E+10,1,67E+06 $726,1.00 \mathrm{E}+10,1.67 \mathrm{E}+06$.
0.. 0., g, Qe+00, 0., Lindane

$0 . .0 ., 5.70 e+01,0 ., 124 t r i m e t h y l b$

$0 ., 0,5,90 e+03,0,0$ lhexatiol

$0 ., 0,5.90 e+03,0 .$, 2hexartone

$0 ., 0.6 .13 \mathrm{~B}+01,0 .$, butylbenzene

-- Input File: RQSITE.DAT

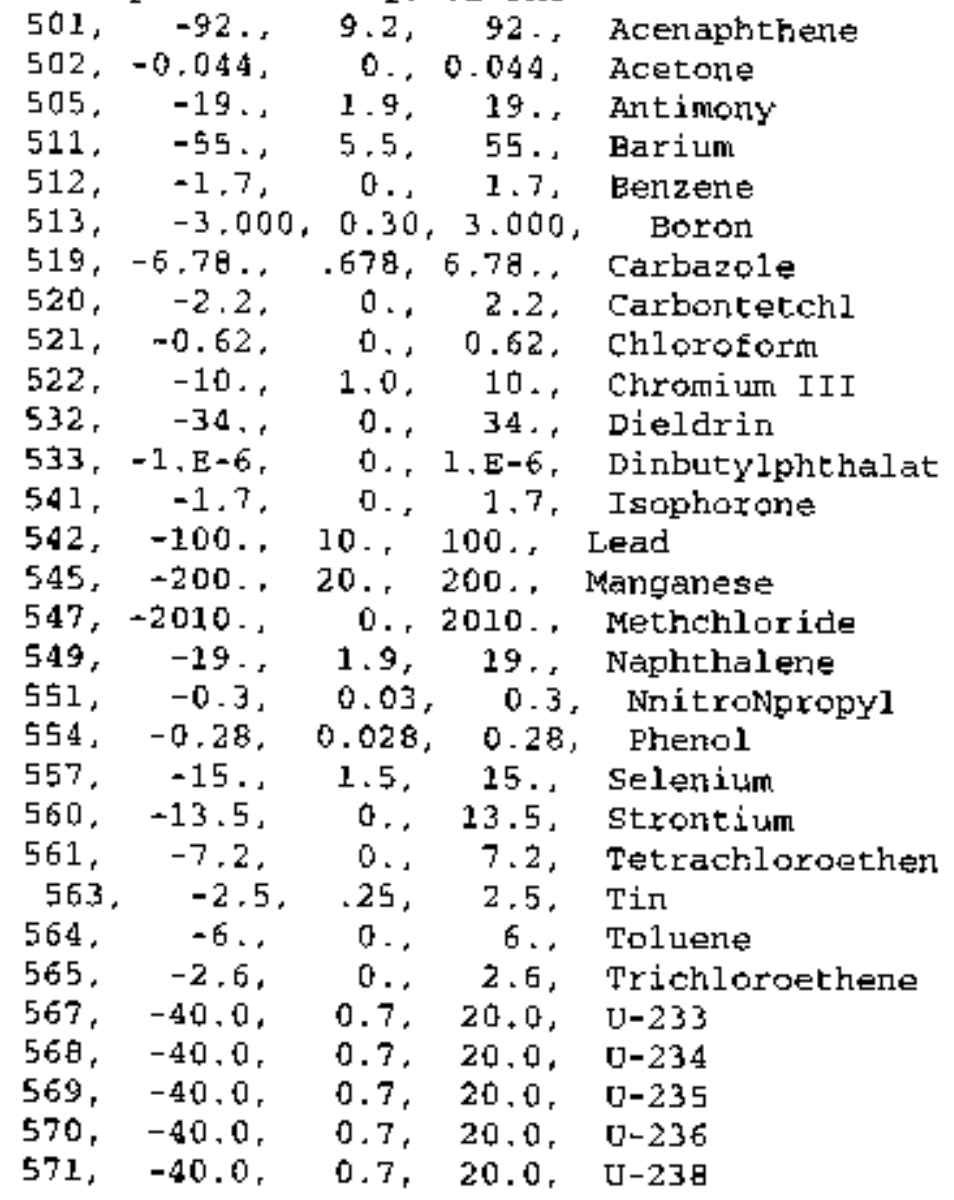




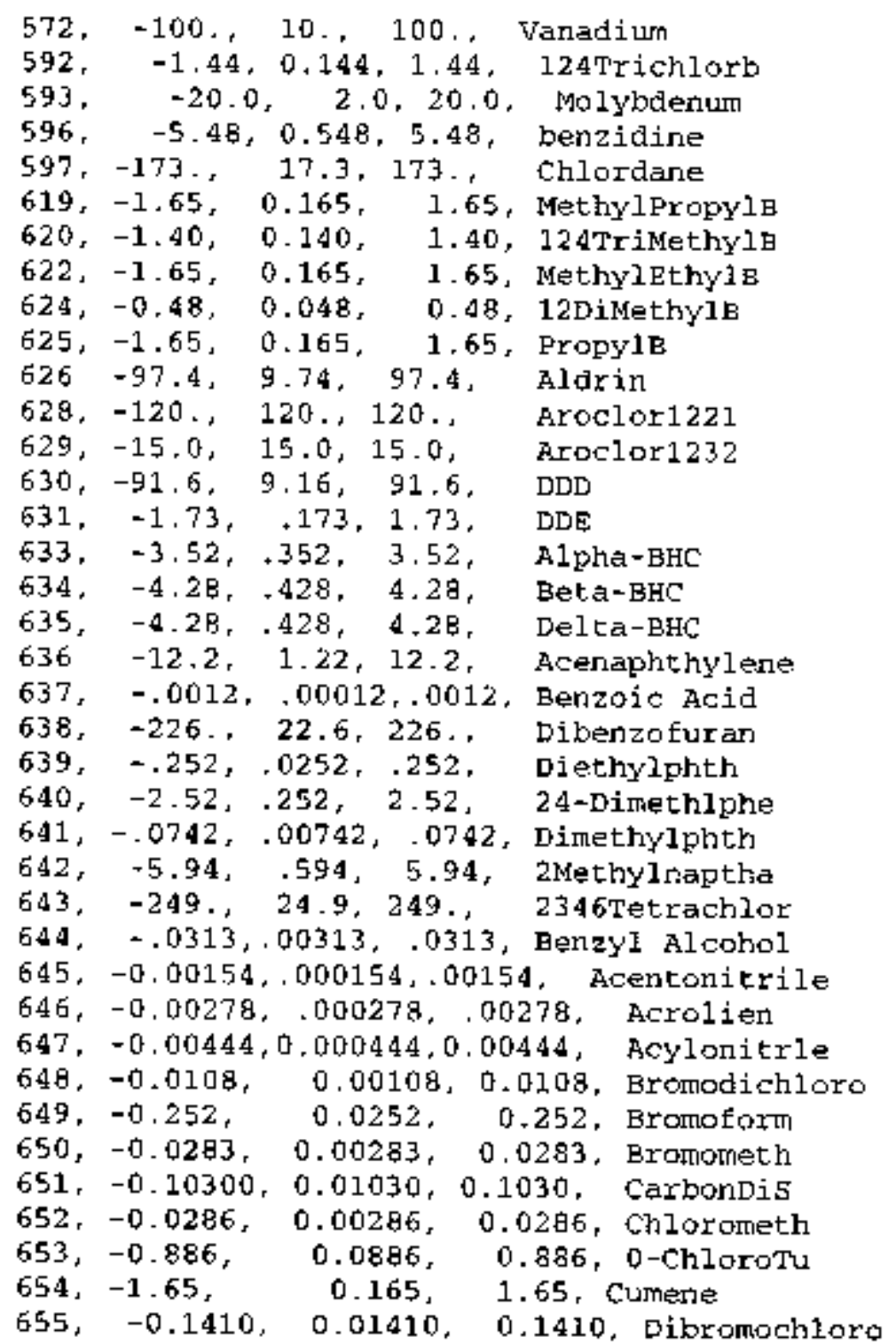




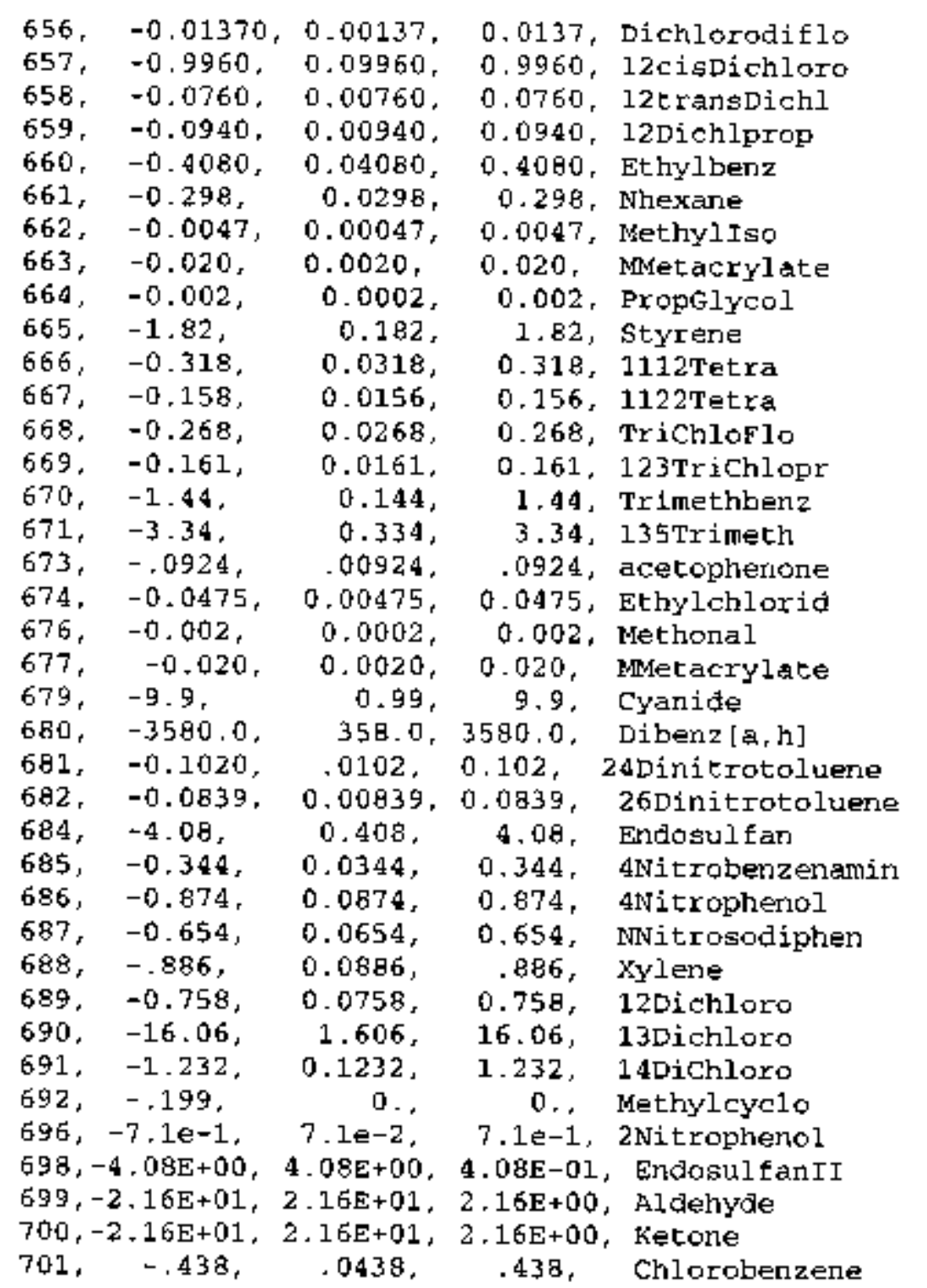




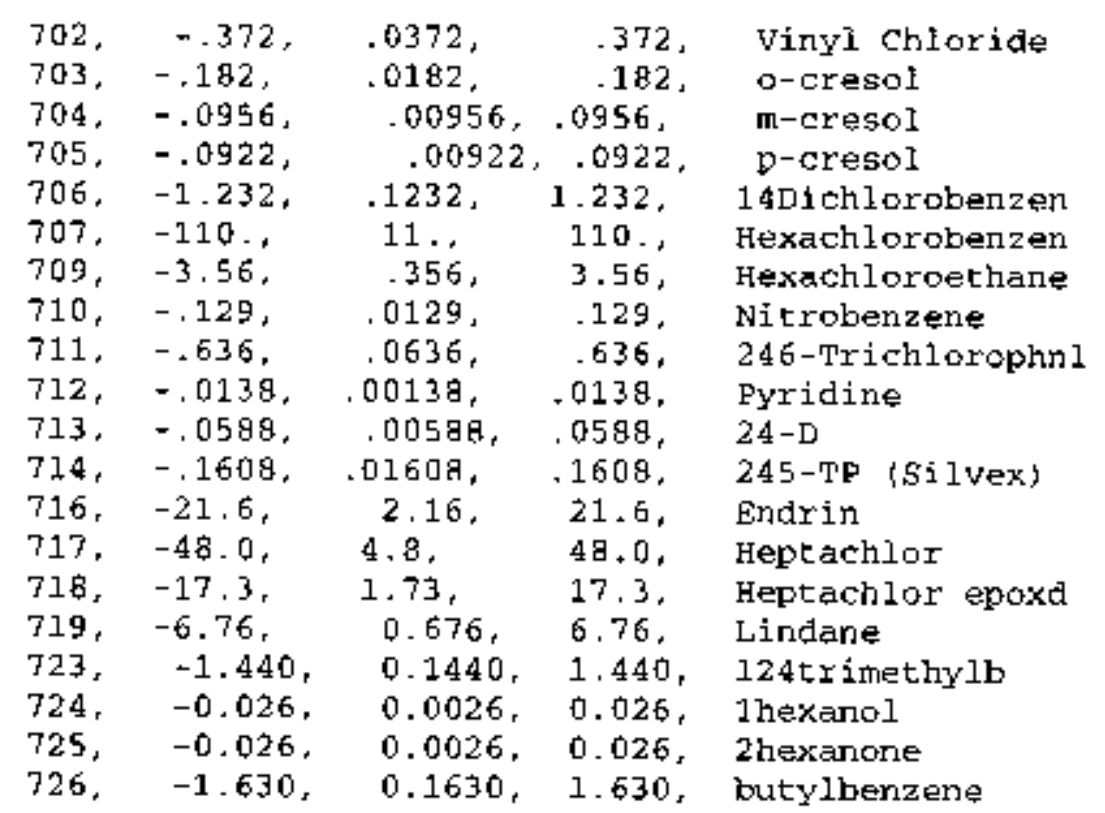

-- Input File: UPTAKE.DAT

$0.5, \quad 0.2, \quad 1.89$

$0.67,0.65,2.1 E-3,438 ., 438$.

0.0. 2160., 24.. 1440., $1 ., 0.83$

50., 6., 48.. 480.. 48 .

$.05,0.0008,60 ., \quad 8 ., 50$.

10., 176., 110., 0., 95., 730..6.9

Acenaphthene $\quad .25,1.2 \mathrm{e}-1,1.2 \mathrm{e}-2,1.6 \mathrm{e}-4,0 ., 5.0 \mathrm{e}-4,1.1 \mathrm{e}+3$

Bcetone $\quad 25,1.3 e+1,1.3 e+0,1.5 \mathrm{E}-9,0 ., 1.5 \mathrm{E}-8,1.5 \mathrm{E}-8$

Antimony $\quad 25,5.0 e-2,5.0 e-3,2.5 e-5,0,4.0 e-5,1.0 e+2$

Bariurn $\quad 25,1.0 \mathrm{e}-1,1.0 \mathrm{E}-2,4.8 \mathrm{E}-4,0,2.0 \mathrm{e}-4,0.0 \mathrm{e}+0$

Benzene $\quad 25,5.8 \mathrm{E}-1,5.9 \mathrm{E}-2,3.3 \mathrm{E}-6,0 ., 3.3 \mathrm{E}-6,3.3 \mathrm{E}-6$

$\begin{array}{llll}\text { Boron } & 25,4.0 \mathrm{e}+0,4.0 \mathrm{e}-1,1.5 \mathrm{e}-3, & 0 ., 8.0 \mathrm{e}-4,0.0 \mathrm{e}+0 \\ \text { Carbazole } & 25,2.4 \mathrm{e}-1,2.4 \mathrm{e}-2,5.0 \mathrm{e}-5,0,1.6 \mathrm{e}-4,4.5 \mathrm{e}+2\end{array}$

carbontetchl $25,2.9 \mathrm{e}-1,2,9 \mathrm{e}-2,1.1 \mathrm{e}-5,0 ., 1.1 \mathrm{e}-5,1.1 \mathrm{e}-5$

Chloroform $\quad 25,7.0 \mathrm{e}-1,7.0 \mathrm{e}-2,2.3 \mathrm{e}-6,0.2 .3 \mathrm{e}-6,2.3 \mathrm{e}-6$

Chromium III $\quad 25,4.0 \mathrm{e}-2,4.0 \mathrm{E}-3,1.0 \mathrm{e}-5,0 ., 9.0 \mathrm{e}-3,2.0 \mathrm{e}+2$ 


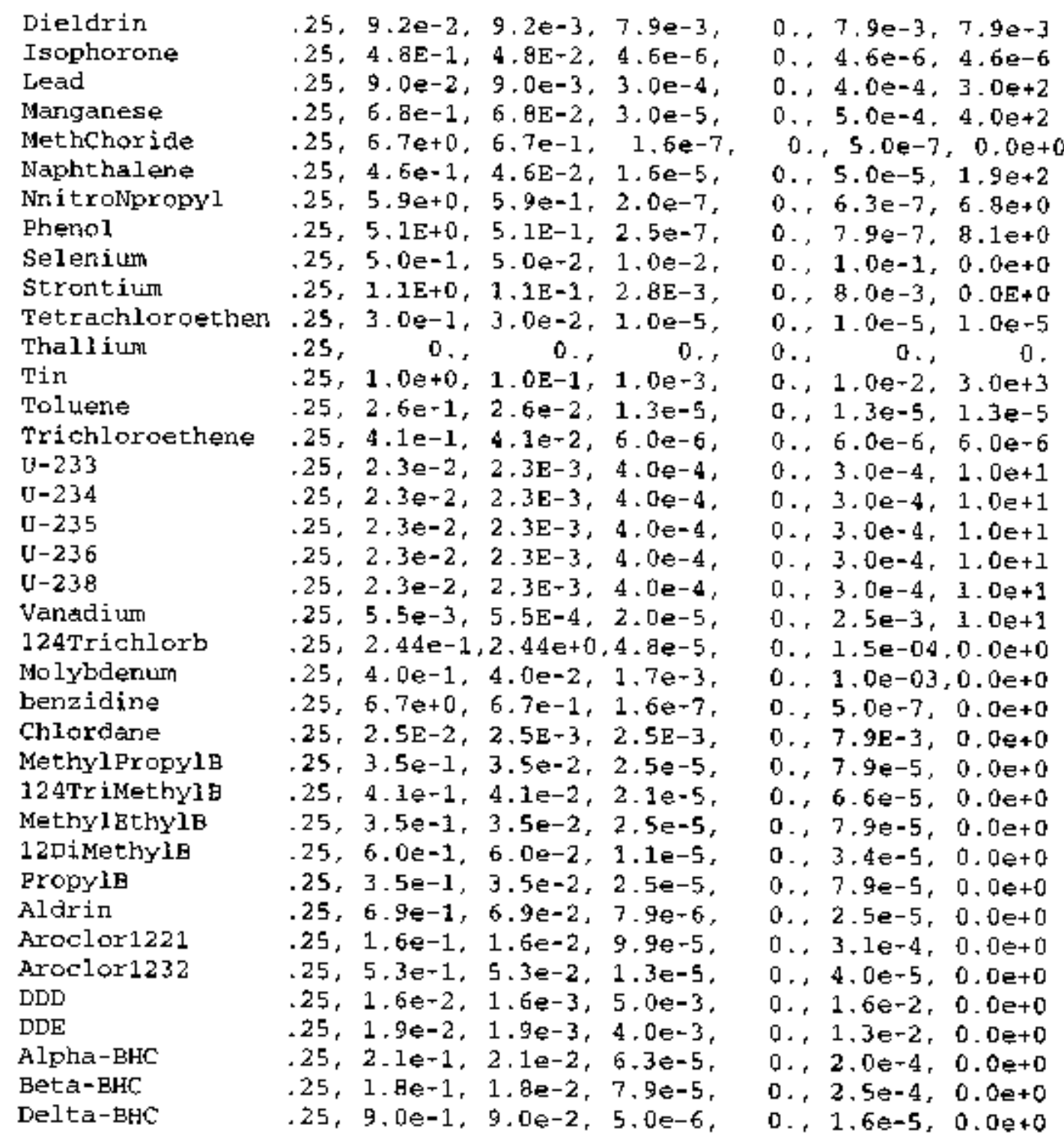




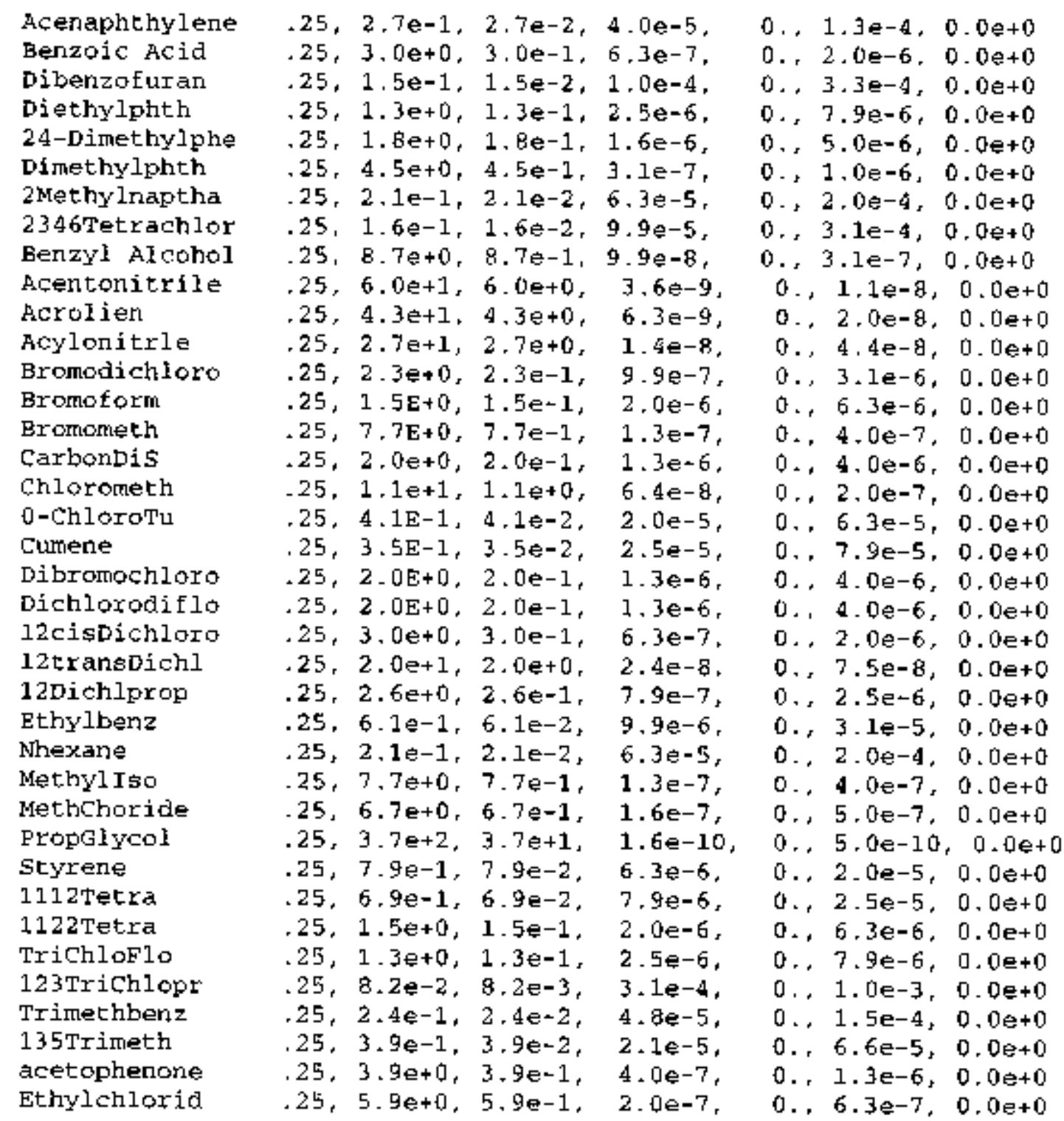




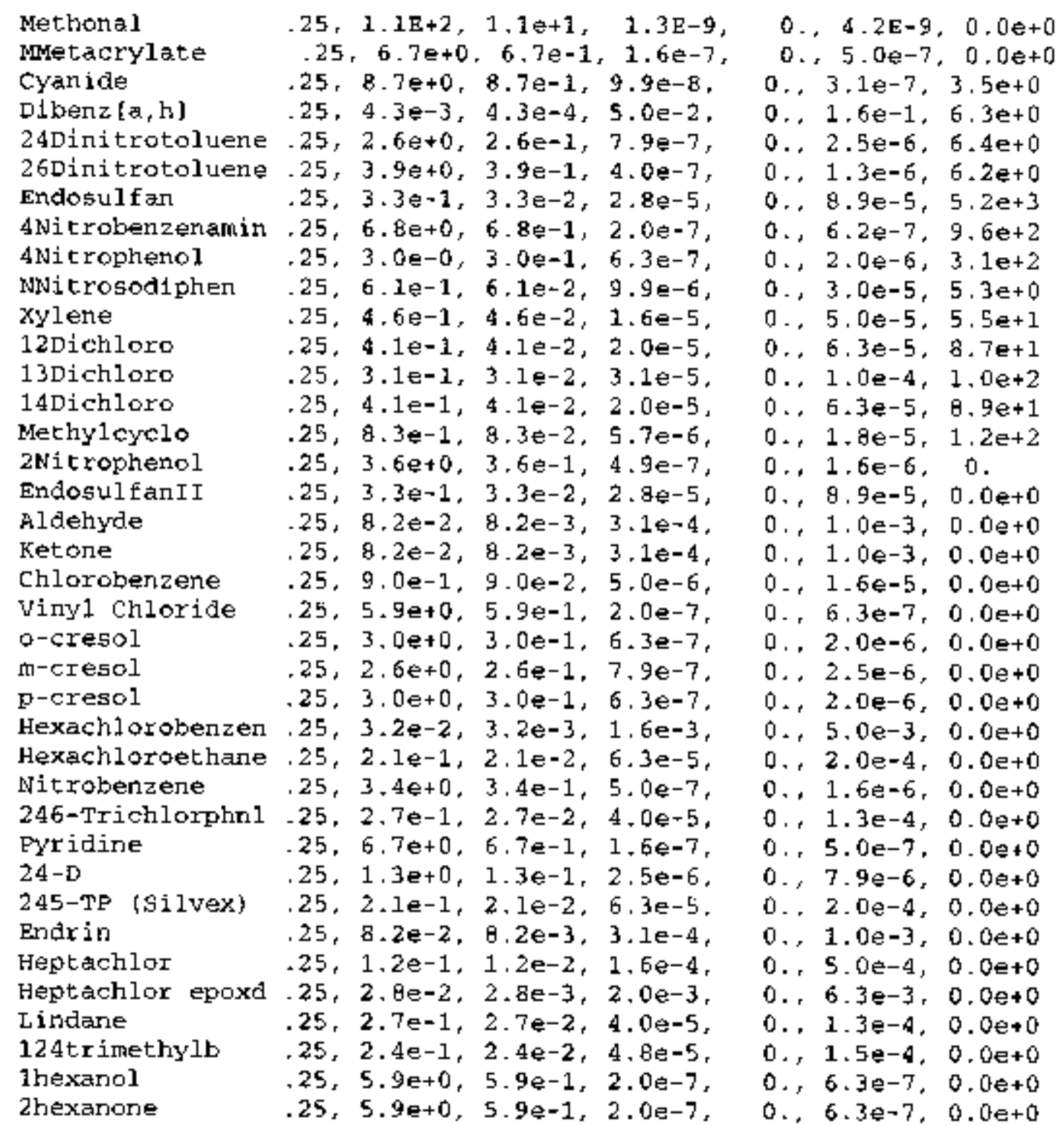


butylвелzепе $\quad 25,3.5 e-1,3.5 e-2,2.5 e-5, \quad 0,7,9 e-5,0.0 e+0$

THERE ARE 202 CONTAMINANTS IN THE RISK EACTOR LIBRARY THE VALUE OF IFLAG IS 0

NUMBER OF PATHWAYS IS 5

PATHWAY

TYPE OF USAGE

FOR UPTAKE FACTORS

$\begin{array}{lll}1 & \text { GROCNDWATER TO RIVER } & 2 \\ 0 & 3 \mathrm{X}, I 2,2 \mathrm{X}, \mathrm{A} 22,6 \mathrm{X}, I 2\}) & 0 \\ 0 & 3 \mathrm{X}, I 2,2 \mathrm{X}, \mathrm{A} 22,6 \mathrm{X}, I 2\}) & 0 \\ 0 & 3 \mathrm{X}, I 2,2 \mathrm{X}, \mathrm{A} 22,6 \mathrm{X}, I 2\}) & 0 \\ 0 & 3 \mathrm{X}, I 2,2 \mathrm{X}, \mathrm{A} 22,6 \mathrm{X}, I 2\} & 0\end{array}$

TIME OF OPERATION OF WASTE FACILITY IN YEARS 0 .

LENGTH OF REPOSITORY (M)

WIDTH OF REPOSITORY (M)

RIVER FLOW RATE $\left(M^{* * 3 / Y R)}\right.$

STREAM FLOW RATE (M**3/YR)

DISTANCE TO RIVER (M)

OPERATFONAL SPILLAGE FRACTION

DENSFTY OF AQUIFER (KG/M**3)

LONGFTUDINAL DISPERSTVITY (M)

LATERAL DISPERSION COEFPICIENT -- Y AXIS [M* $2 / Y R$ )

NUMEER OF MESH POTNTS FOR DISPERSION CALCULATION

FLAG FOR ATMOSPHERFC PATHWAY

COVER THICKNESS OVER WASTE (M)

THICKNESS OF WASTE IN PITS (M)

TOTAL WASTE VOLUME $\left(M^{* * 3}\right)$

DISTANCE TO WELL - $X$ COORDINATE (M)

DIGTANCE TO WELL - Y Y COORDINATE (M)

DENSITY OF WASTE (KG/M**3)

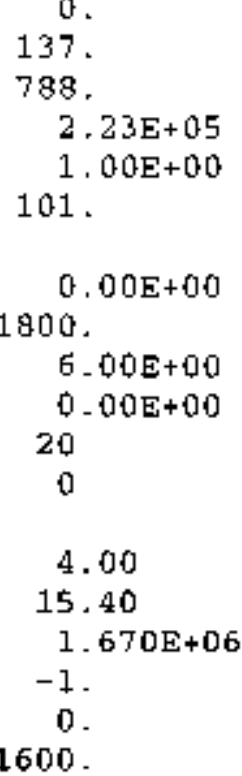

1600 . 
FRACTION OF FOOD CONSUMED THAT IS GROWN ON SITE FRACTION OF YEAR CONTAMINANTS CONTACT SKIN

AREA OF SKIN IN CONTACT WITH CONTAMINANTS $\left(\mathrm{M}^{* * 2}\right.$ ) DEPTH OF PLANT ROOT ZONE (M)

AREAL DENSITY OF PLANTS \{KG/M**2\}

AVERAGE DUST LOADING IN AIR \{ $\mathrm{KG} / \mathrm{M}^{* * 3}$ \}

ANNUAL ADULT BREATHING RATE \{M**3/YR\}

FRACTION OF YEAR EXPOSED TO DUST

CANISTER LIFETTIME (YEARS\})

INUENTORY SCALING FACTOR

HEIGHT OF ROOMS IN RECLAIMER HOUSE (CM)

AIR CHANGE RATE IN RECLAIMER HOUSE (CHANGES/SEC)

ATMOSPHERIC STAEILITY CLASS

AVERAGE WIND SPEED (M/S)

FRACTION OF TIME WIND GLOWS TOWRPD RECEPTOR

RECEPTOR DISTANCE FOR ATMOSPHERIC FATHWAY (M)

DUST RESUSPENSION RATE FOR OFFSITE TRANSPORT $\left\{\mathbb{A}^{* * 3 / 3}\right\}$

DEPOSITION VELOCITY (M/S)

\$TACK HEIGHT \{M\}

STACK INSIDE DIAMETER (M)

STACK GAS VELOCITY (M/S)

HEAT EMISSION RATE FROM BURNING (CAL/S)

FLAGS FOR DEGRADATION SERIES

00

FLAG FOR INPUT SUMMARY PRINTOUT

FTAG FOR DIRECTION OF TREHFH FILLING

FLAG FOR GROUNDWATER PATHWAY OPTIONS

ANOUNT OF WATER PERCOLATING THROUGH WASTE ANNUALLY (M)

DEGREE OF SOIL SATURATION

RESIDUAI SOIL SATURATION

$$
\begin{aligned}
& .400 \\
& .705 \\
& .0100 \\
& .900
\end{aligned}
$$

1.000

1.00E-07

8000.

.705

0 .

1. $00 \mathrm{OE}+0.0$

240 .

$5.56 \mathrm{E}-0.4$

\section{4}

6.30

.2300

, 0

1. $10 \mathrm{E}-06$

.0100

.0

00

.0

$0.00 \mathrm{E}+00$

0

1

0

$9.10 \mathrm{E}-03$

1. 000

$-000$

.32

.000

.04

POROSITY OF AQUIFER

POROSITY OF UNSATURATED ZONE

.25 
DISTANCE FROM AQUIFER TO WASTE (M)

AVERAGE VERTICAL GROUNDWATER VELOCITY \{M/YR\}

HORIZONTAL VELOCITY OF AQUIFER (M/YR)

LENGTH OF PERFORATED WELL CASING (M)

SURFACE EROSION RATE (M/YR)

LEACH RATE SCALING FACTOR

ANNUAL RUNOFF OF PRECIPITATION (M)

**** PEAK CONCENTRATIONS AND TIMES FOR PATHWAY $1 * \psi * *$

$* * * *$ RIVER AT $101.0 \mathrm{M} * * * * *$

ConTAmINANT
Acenaphthene
Acetone
Antimony
Earium
Benzene
Boran
Carbazole
Carbontetchl
Chloroform
Chromium II
Dieldrin
Isophorone
Lead
Manganese
Naphthalene
Nnitronpropyl

PEAR

CONCENTRATION (MG/L)
1. $51 \mathrm{E}-02$
1. $38 \mathrm{E}+01$
1. $44 \mathrm{E}-01$
5. $01 \mathrm{E}-02$
1. $49 \mathrm{E}+00$
$6.95 \mathrm{E}-01$
7. $93 \mathrm{E}-03$
1. $17 \mathrm{E}+00$
$3,56 \mathrm{E} 400$
2. $72 \mathrm{E}-01$
8. 10E-02
1. $49 \mathrm{E}+00$
$2.70 \mathrm{~B}-02$
$1.35 E-02$
1. $41 E-01$
6. $05 E+00$

7.2

2. $50 \mathrm{E}-02$

$4.24 E+100$

10.000

1. $000 \mathrm{E}-05$

l. $000 \mathrm{E}+00$

$0.00 \mathbf{E}+00$
PEAK TIME

250605.0

603.4

52064.3

149974.7

4777.3

10258.2

22594.9

6067.9

1989.6

33104.0

88150.1

4777.3

536630.9

1072735.7

102504.4

2270. 2
AVERAGE DOSE

4. $34 \mathrm{E}-04$

3. $96 \mathrm{E}-01$

4. 14E-03

1. $45 \mathrm{E}-03$

4. 27 E-02

$2.03 \mathrm{E}-02$

2. $28 \mathrm{E}-04$

3. 37E-02

1. $02 \mathrm{E}-01$

B. $30 E-03$

2. $63 \mathrm{E}-03$

4. $27 \mathrm{E}-02$

7. $78 \mathrm{E}-04$

3. $88 \mathrm{E}-04$

4. $05 \mathrm{E}-03$

1. $74 \mathrm{E}-01$
AVERAGE RISK

AT PEAK TIME

(HE/LIFE)

0. $00 \mathrm{E}+00$

$0.00 \mathrm{E}+00$

$0.00 E+00$

$0.00 \mathrm{E}+00$

1. $24 \mathrm{E}-03$

0. $00 \mathrm{E}+00$

4. $56 \mathrm{E}-06$

$4.38 \mathrm{E}-03$

6. $23 \mathrm{E}-04$

0. $0 \mathrm{E}+00$

3. $41 E-02$

$4,06 \mathrm{E}-05$

$0.00 E+00$

$0.00 \mathrm{E}+00$

$0.00 \mathrm{E}+00$

1. $22 \mathrm{E}+00$
FRACTION

OF ADI

7. 24E-04

3. $96 \mathrm{E}+00$

1. $04 \mathrm{E}+01$

2. $07 \mathrm{E}-02$

o. $00 \mathrm{E}+00$

1. $02 \mathrm{E}=01$

$0.00 \mathrm{E}+00$

4. $81 \mathrm{E}+01$

1. $02 E+01$

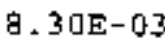

$5.25 E+101$

2. 14E-01

5. $56 \mathrm{E}-01$

8. $27 \mathrm{E}-03$

1.13E-01

$0.00 E+00$ 


$\begin{array}{ll}\text { Phenol } & 6.33 \mathrm{E}+00 \\ \text { Selenium } & 1.79 \mathrm{E}-01 \\ \text { Strontium } & 2.03 \mathrm{E}-01 \\ \text { Tetrachloroethen } & 3.76 \mathrm{E}-01 \\ \text { Tin } & 1.02 \mathrm{E}+00 \\ \text { Toluene } & 4.49 \mathrm{E}-01 \\ \text { Trichloroethene } & 1.00 \mathrm{E}+00 \\ \text { U-233 } & 5.11 \mathrm{E}-02 \\ \text { U-234 } & 5.65 \mathrm{E}-02 \\ \text { U-235 } & 6.89 \mathrm{E}-02 \\ \text { U-236 } & 6.87 \mathrm{E}-02 \\ \text { U-238 } & 6.89 \mathrm{E}-02 \\ \text { Vanadium } & 2.70 \mathrm{E}-02 \\ \text { 124Trichlorb } & 2.51 \mathrm{E}-01 \\ \text { Molybdentim } & 1.37 \mathrm{E}-01 \\ \text { Benzidine } & 4.91 \mathrm{E}-01 \\ \text { Chlordane } & 2.47 \mathrm{E}-04 \\ \text { MethylPropylB } & 2.69 \mathrm{E}-01 \\ \text { MethylEthylE } & 2.69 \mathrm{E}-01 \\ \text { 12DiMethys } & 9.69 \mathrm{E}-01 \\ \text { PropylB } & 2.69 \mathrm{E}-01 \\ \text { Aldrin } & 7.49 \mathrm{E}-05 \\ \text { Aroclor1221 } & 2.10 \mathrm{E}-02 \\ \text { Aroclor1232 } & 2.13 \mathrm{E}-02 \\ \text { DDD } & 3.96 \mathrm{E}-04 \\ \text { DDE } & 1.76 \mathrm{E}-04 \\ \text { Alpha-BHC } & 3.52 \mathrm{E}-02 \\ \text { Beta-BHC } & 3.52 \mathrm{E}-02 \\ \text { Delta-BHC } & 3.52 \mathrm{E}-02 \\ \text { Acenaphthylene } & 7.09 \mathrm{E}-02 \\ \text { Benzoic Acid } & 1.50 \mathrm{E}+01 \\ \text { Dibenzofuran } & 1.22 \mathrm{E}-02 \\ \text { Diethylphth } & 4.76 \mathrm{E}+00 \\ \text { 24-Dimethylphe } & 1.03 \mathrm{E}+00 \\ \text { Dimethylphth } & 1.20 \mathrm{E}+01 \\ \text { Dinbutylphthalat } & 1.77 \mathrm{E}+01 \\ \text { 2Methylnaptha } & 1.08 \mathrm{E}-01 \\ \text { 2346Tetrachlor } & 1.11 \mathrm{E}-02 \\ & \end{array}$

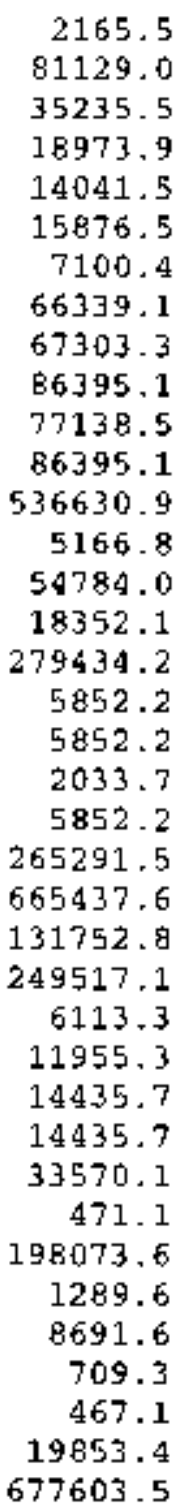

677603.5

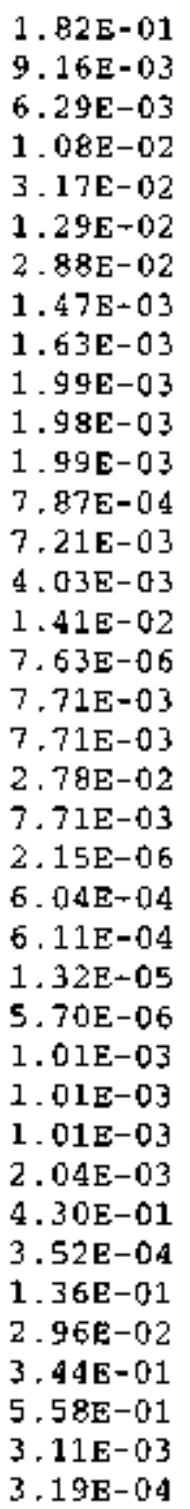
$3.03 E-01$
1. $83 \mathrm{E}+00$
1. $05 \mathrm{E}-02$
1. $O B E+00$
5. $28 \mathrm{E}-02$
6. $44 \mathrm{E}-02$
$0.0 O E+O O$
4. $91 \mathrm{E}-01$
$5.43 \mathrm{E}-01$
$6.62 \mathrm{E}-01$
$6.61 \mathrm{E}-01$
6. $62 E-01$
$1.12 \mathrm{E}-01$
1. $44 \mathrm{E}-01$
ล. $05 E-01$
$4.69 \mathrm{E}+00$
1. $53 \mathrm{E}-02$
2.08E-01
2. 0 GE-01
1. 39E-02
2. 08E-01
7. 16 E-02
0. $00 \mathrm{E}+00$
$0.00 \mathrm{E}+00$
0. $00 \mathrm{E}+00$
$0,00 E+00$
0. $00 \mathrm{E}+00$
$0.00 \mathrm{E}+00$
$0.00 \mathrm{E}+00$
3. $39 \mathrm{E}-02$
1. $07 \mathrm{E}-01$
8. $80 \mathrm{E}-02$
1. $71 \mathrm{E}-01$
$1.48 \mathrm{E}+00$
3. $44 \mathrm{E}-02$
$5.58 \mathrm{E}+00$
$7.79 \mathrm{E}-01$
1. $06 \mathrm{E}-02$ 
Benzyi Aicohol Acentonitrile Acrolien

Acylonitrie

Bromodichloro

Bromoform

Bromometh

Carbondis

Chlorometh

o-Chlorotu

cumene

Dibromoch1oro

Dichlorodiflo

12cisDichloro

12 transDich 1

12Dichlprop

Ethylbenz

Nhexane

Methyl Iso

MethChoride

propulycol

styrene

lli2Tetra

$1122 \mathrm{Tetra}$

Trichloflo

123Trichlopr

Trimethbenz

135Trimeth

acetopherone

Ethylchlorid

Methona 1

Metacrylate

Cyanide

Dibenz [a.h]

24Dinitrotoluene

26Dinitrotoluene

Endosulf ar

4Nitrobenzenamin

\begin{tabular}{|c|c|}
\hline $2.47 \mathrm{E}+0 \mathrm{I}$ & 569.3 \\
\hline $2.34 \mathrm{E}+01$ & 472.2 \\
\hline $2.32 \mathrm{E}+0 \mathrm{I}$ & 476.2 \\
\hline $2.30 E+01$ & 481.6 \\
\hline $1.78 E+01$ & 502.4 \\
\hline $9.05 E+00$ & 1289.6 \\
\hline $2.00 E+01$ & 559.5 \\
\hline $6.94 E+00$ & 903.3 \\
\hline $2.00 \mathrm{E}+01$ & 560.5 \\
\hline $2.20 \mathrm{E}+00$ & 3358.8 \\
\hline $1.97 \mathbf{E}-01$ & 50976.4 \\
\hline $1.24 \mathrm{E}+01$ & 927.3 \\
\hline $1.65 \mathbf{E}+60$ & 511.9 \\
\hline $3.21 E+00$ & 3717.8 \\
\hline 1. $59 \mathrm{E}+01$ & 715.2 \\
\hline $1,4 \mathrm{gE}+01$ & 773,9 \\
\hline $9,94 E-01$ & 1798, 7 \\
\hline $5.59 \mathrm{E}-02$ & 1439.7 \\
\hline $2.29 \mathrm{E}+01$ & 482.5 \\
\hline $2.10 E+01$ & 532.4 \\
\hline $2.33 E+01$ & 473.7 \\
\hline 1. $82 \mathrm{E}+00$ & 6407.0 \\
\hline $6.29 \mathrm{E}+00$ & 1505.0 \\
\hline 1. $18 \mathrm{E}+01$ & 976.3 \\
\hline $6.47 \mathrm{E}+\mathrm{DO}$ & 1341.8 \\
\hline $1.03 \mathrm{E}+01$ & 992.6 \\
\hline $3.35 \mathrm{E}-01$ & 5166.8 \\
\hline $2,84 \mathrm{E}-01$ & 11367.8 \\
\hline 1. $49 \mathrm{E}+01$ & 768.7 \\
\hline 1. $81 \mathrm{E}+01$ & 622.2 \\
\hline $2.33 \mathrm{E}+01$ & 473.7 \\
\hline 1. $5.7 \mathrm{E}+01$ & 532.4 \\
\hline \multirow[t]{2}{*}{$2.75 \mathrm{E}-01$} & 32777.6 \\
\hline & $>1000000.0$ \\
\hline $1.19 E+00$ & 900.0 \\
\hline $1.55 \mathrm{E}+60$ & 741.0 \\
\hline $1.98 \mathrm{E}-03$ & 13793.0 \\
\hline $4.71 \mathrm{E}-08$ & \\
\hline
\end{tabular}

$0.005+00$

$0.00 E+00$

$0.00 E+00$

3. $55 \mathrm{E}-01$

3. $17 \mathrm{E}-02$

2. $05 \mathrm{E}-03$

$0.00 \mathrm{E}+00$

$0.00 \mathrm{E}+00$

$7.45 \mathrm{E}-03$

$0.00 E+00$

D. $00 \mathrm{E}+00$

2. $99 \mathrm{E}-02$

$0.00 \mathrm{E}+00$

$0.00 \mathrm{E}+00$

$0.00 \mathrm{E}+00$

$2.88 \mathrm{E}-02$

$0.00 \mathrm{E}+00$

$0.00 E+00$

$0.00 E+00$

4. $51 E-03$

$0.00 \mathrm{E}+00$

$0.00 E+00$

4. $69 \mathrm{E}=03$

$6.74 \mathrm{E}-02$

$0.00 E+00$

$2,09 \mathrm{E}+00$

$0.00 \mathrm{E}+00$

$0.00 \mathrm{E}+00$

$0.00 \mathrm{E}+00$

1. $51 \mathrm{E}-03$

$0.00 E+00$

$0.00 E+00$

$0,00 \mathrm{E}+00$

$2.32 \mathrm{E}-02$

3. $02 \mathrm{E}-02$

$0.00 \mathrm{E}+00$

2.84E-11
$1.41 \mathrm{E}+00$

1. $12 \mathrm{E}+02$

1. $33 \mathrm{E}+03$

6. $59 \mathrm{E}+02$

2. $56 \mathrm{EE}+01$

1. $30 \mathrm{E}+01$

$4,10 E+02$

$1.99 \mathrm{E}+00$

$0.00 \mathrm{E}+00$

$3.16 \mathrm{E}+0 \mathrm{O}$

$5.65 \mathrm{E}-02$

1. $7 \mathrm{BE}+\mathrm{DI}$

$2,36 \mathrm{E}-01$

9. $29 \mathrm{E}+00$

2. $28 \mathrm{E}+01$

$0.00 \mathrm{E}+00$

2. $85 \mathrm{E}-01$

$2.68 \mathrm{E}-02$

$8.23 \mathrm{E}+00$

1. $00 \mathrm{~B}+01$

1. $34 \mathrm{~B}+00$

$2.62 \mathrm{E}-01$

$6.02 \mathrm{E}+00$

$5.62 \mathrm{E}+00$

$6.19 \mathrm{E}-01$

$4.97 \mathrm{E}+01$

1. $93 \mathbf{E}=01$

$1.63 \mathrm{E}-01$

$4.26 \mathrm{E}+00$

1. $30 \mathrm{E}+00$

1. $34 \mathrm{E}+00$

3. $21 \mathrm{E}-01$

3. $94 \mathrm{E}-01$

$1-71 E+01$

4. $45 E+01$

9. $46 \mathrm{E}-03$

$4.51 \mathrm{E}-07$ 
4 Ni trophenol

NNi trosodiphen

XYlene

12Dichloro

13Dichloro

14Dichloro

Methylcyclo

2Nitrophenol

EndosulfanI $\mathrm{I}$

Aldehyde

Ketone

Chlorobenzene

vinyl chloride

o-cresol

m-cresol

p-cresol

Hexachlorobenzen

Hexachloroethane

Nitrobenzene

246-Trichlorphnl

Fyridine

24-D

245-TP (Silvex)

Endrin

Heptachlor

Heptachlor epoxd

Iindane

124trimeth $\gamma$ lb

lhexanol

2hexanone

butylbenzene

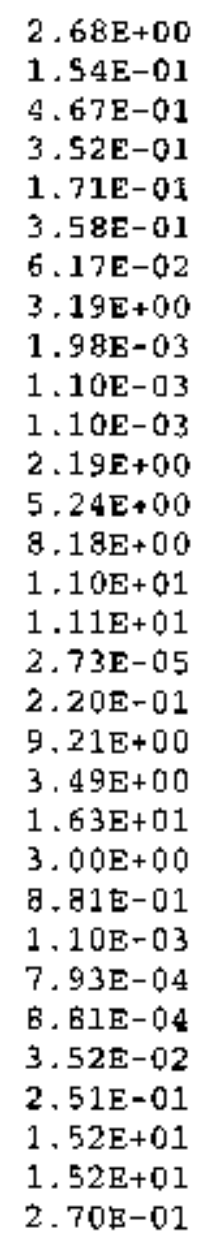

7. $70 \mathrm{E}-02$

4. $42 \mathrm{E}-03$

1. $34 \mathrm{E}-02$

1. $01 \mathrm{E}-02$

4. $90 \mathrm{E}-03$

1. $03 \mathrm{E}-02$

1. $77 \mathrm{E}-0.3$

9. $16 \mathrm{E}-02$

5. $69 \mathrm{E}-05$

3. $19 \mathrm{E}-05$

3. $19 \mathrm{E}-05$

6. $-29 \mathrm{E}-02$

1. $50 \mathrm{E}-01$

2. 35E-01

3. $15 \mathrm{E}-01$

3. $19 \mathrm{E}-01$

8. $23 \mathrm{E}-07$

$6.33 \mathrm{E}-03$

$2.64 \mathrm{E}-01$

1. $00 \mathrm{E}-01$

4. $66 \mathrm{~B}-01$

8. $62 \mathrm{E}-02$

$2.53 \mathrm{E}-02$

3. 19E-05

$2.29 \mathrm{E}-05$

2. $69 \mathrm{E}-05$

1. $01 \mathrm{E}-03$

7. 21E-03

4. 35E-01

4. $35 \mathrm{E}-01$

$7.75 \mathrm{E}-03$
$0.00 \mathrm{E}+00$

2. $17 \mathrm{E}-05$

$0.00 E+00$

$0.00 E+00$

$0.00 \mathrm{E}+00$

2. $47 E-04$

$0.00 \mathrm{E}+00$

$0.00 \mathrm{E}+00$

$0,00 E+00$

$0.00 \mathrm{E}+00$

$0.00 \mathrm{E}+00$

$0.00 \mathrm{E}+00$

2. $10 \mathrm{E}-01$

$0.00 \mathrm{E}+00$

$0.00 E+D D$

0. $.00 E+00$

1. 32 E-06

8. 86E-05

$0.00 \mathrm{E}+00$

1. $10 \mathrm{E}-03$

$0.00 \mathrm{E}+00$

c. $00 E+00$

0. $00 \mathrm{E}+00$

$0.00 \mathrm{E}+00$

1. 03 E-04

2. 44E-04

1. $32 E-03$

0. $00 \mathrm{~B}+00$

$0.00 \mathrm{E}+00$

D.

1. $00 \mathrm{E}+00$
1. $24 E+00$

2. $21 \mathrm{E}-01$

6. 70E-02

1 , 12E-01

5. $50 \mathrm{E}-02$

4. 47E-02

2. $95 \mathrm{E}-02$

1. 4 白 $\mathrm{E}+00$

9. 4 BE-03

1. $06 \mathrm{E}-01$

1. $06 \mathrm{E}-01$

3. $15 \mathrm{E}+00$

5. $01 \mathrm{E}+01$

4. $69 \mathrm{E}+00$

6. $30 \mathrm{E}+00$

6. $39 \mathrm{E}+01$

1. $03 \mathrm{E}-03$

6. $33 \mathrm{E}+00$

5. $28 \mathrm{E}+02$

o. $00 \mathrm{E}+00$

4. $66 \mathrm{E}+02$

B. $62 \mathrm{E}+00$

3. $16 \mathrm{E}+00$

1. $06 \mathrm{E}-01$

4. $57 \mathrm{E}-02$

2. $07 \mathrm{E}+00$

$3.37 E+00$

1. $44 E-01$

1. $09 \mathrm{E}+01$

1. $09 \mathrm{E}+01$

2. . 4 4E-O1 
GROUNDWATER TO RIVER

$* * * * * * * * *$ CONTAMINANT DOSES (mg/kg-day $t * t * * * *$

\begin{tabular}{|c|c|c|}
\hline TIMES & 1000 & 100000 \\
\hline Aceraphthene & $0.0 \mathrm{E}+00$ & $0.0 \mathrm{E}+00$ \\
\hline Acetone & $3.2 \mathrm{E}-03$ & $0.0 \mathrm{E}+00$ \\
\hline Antimony & $0.0 \mathrm{~B}+00$ & $2.1 \mathrm{E}-06$ \\
\hline Barium & $0.0 \mathrm{~B}+00$ & $0.0 E+00$ \\
\hline Benzene & $0.0 \mathrm{E}+00$ & $0.0 E+00$ \\
\hline Boron & $0.0 \mathrm{E}+00$ & $0.0 \mathrm{E}+00$ \\
\hline Carbazole & $0.0 E+00$ & $2.3 E-04$ \\
\hline Carbontetchl & $0.0 \mathrm{E}+00$ & $0.0 \mathrm{E}+00$ \\
\hline ch1oroform & $0.0 E+00$ & $0.0 \mathrm{E}+0 \mathrm{O}$ \\
\hline chromium III & $0.0 E+00$ & $0.0 \mathbf{E}+0.0$ \\
\hline Dielorin & $0.0 \mathrm{E}+00$ & $2.6 \mathbf{E}-0.3$ \\
\hline I sophorone & $0.0 \mathrm{E}+00$ & $0.0 \mathrm{E}+0.0$ \\
\hline Lead & $0.0 \mathrm{E}+00$ & $0.0 \mathrm{E}+00$ \\
\hline Manganese & $0.0 \mathbf{E}+00$ & $0.0 \mathrm{E}+00$ \\
\hline Naphthalene & $0.0 \mathrm{E}+00$ & $4.0 \mathrm{E}-0.3$ \\
\hline Nnitrolupropyl & $1.9 E-07$ & $0.0 E+00$ \\
\hline Phenol & $5.8 \mathbf{E}-05$ & $0.0 E+00$ \\
\hline Seleлium & $0.0 \mathbf{E}+0$ & $4.1 E-03$ \\
\hline strontiun & $0.0 E+00$ & $0.0 \mathrm{E}+00$ \\
\hline Tetrachloroethen & $0.0 \mathrm{E}+00$ & $0.0 \mathrm{E}+00$ \\
\hline Tin & $0.0 \mathrm{E}+00$ & $0.0 E+00$ \\
\hline Toluene & $0.0 E+00$ & $0.0 \mathrm{E}+00$ \\
\hline Trichloroechene & $0.0 E+00$ & $0.0 \mathrm{E}+00$ \\
\hline $\mathrm{U}-233$ & $0.0 E+00$ & $1+3 E-03$ \\
\hline $\mathrm{U}-234$ & $0.0 E+00$ & $1.5 E-03$ \\
\hline $\mathrm{U}-235$ & $0.0 E+00$ & $2.0 E=03$ \\
\hline$(j-236$ & $0,0 E+00$ & 2. $0 \mathrm{E}-03$ \\
\hline $\mathrm{U}-238$ & $0.0 \mathrm{E}+00$ & 2. $0 \mathrm{E}-0.3$ \\
\hline Vanadium & $0.0 \mathrm{E}+00$ & $0.0 \mathrm{E}+00$ \\
\hline $124 \mathrm{Tx}$ jchlorb & D. $0 E+00$ & $0.0 \mathrm{E}+00$ \\
\hline Molybdenum & $0.0 E+00$ & $1.6 \mathrm{E}-03$ \\
\hline benzidine & $0.0 \mathrm{E}+00$ & $0.0 \mathrm{E}+00$ \\
\hline Chlordane & $0.0 E+00$ & $0.0 E+00$ \\
\hline
\end{tabular}




\begin{tabular}{|c|c|c|}
\hline MethylPropylB & $0.0 E+00$ & $0,0 \mathrm{E}+00$ \\
\hline MethylEthylB & $0.0 \mathrm{E}+00$ & $0.0 \mathrm{E}+00$ \\
\hline 12 Dimetbyl $\mathrm{B}$ & $0.0 E+00$ & $0.0 \mathrm{E}+00$ \\
\hline PropylB & $0.0 E+00$ & $0.0 \mathrm{E}+00$ \\
\hline Aldrin & $0.0 \mathrm{E}+00$ & $0.0 \mathrm{E}+00$ \\
\hline Aroclor1221 & $0.0 E+00$ & $0.0 \mathrm{E}+00$ \\
\hline Aroclor 1232 & $0.0 \mathrm{E}+00$ & $6.1 \mathrm{E}-04$ \\
\hline $\mathrm{DDD}$ & $0.0 \mathrm{E}+00$ & $0.0 E+00$ \\
\hline DDE & $0.0 \mathrm{E}+00$ & $5.7 \mathrm{~B}-06$ \\
\hline Alpha-BHC & $0.0 \mathrm{E}+00$ & $1.0 \mathrm{D}-03$ \\
\hline Betr-BHC & $0.0 \mathrm{E}+00$ & $1.0 \mathrm{E}-0.3$ \\
\hline Delta-BHC & $0.0 \mathrm{E}+00$ & $1.0 \mathrm{E}-0.3$ \\
\hline Acenaphthylene & $0.0 \mathbf{E}+00$ & $2.0 \mathrm{E}=03$ \\
\hline Benzoic Acid & 2. $8 \mathbf{E}-12$ & $0.0 \mathrm{E}+00$ \\
\hline Dibenzofuran & $0.0 \mathrm{E}+00$ & $0.0 \mathbf{E}+0.0$ \\
\hline Diethylphth & $1.4 \mathrm{E}=01$ & $0.0 \mathrm{E}+00$ \\
\hline 24-Dimethylphe & $0.0 \mathrm{E}+00$ & $0.0 \mathrm{E}+00$ \\
\hline Dimethylphth & 3. $4 \mathrm{E}-01$ & $0.0 E+00$ \\
\hline 2Methylnaptha & $0.0 \mathrm{E}+00$ & $0.0 \mathrm{E}+00$ \\
\hline 2346 Tetrachlor & $0.0 E+00$ & $0.0 \mathrm{E}+00$ \\
\hline Benzyl Alcohol & 5. $5 E-06$ & $0.0 \mathrm{E}+00$ \\
\hline Acentonitrile & $1.7 E-17$ & $0,0 \mathrm{E}+00$ \\
\hline Acrolien & $6.3 \mathrm{E}-17$ & $0.0 \mathrm{E}+00$ \\
\hline Acylonitrle & $3.5 E-16$ & $0.0 E+00$ \\
\hline Eromodichloro & $6.1 \mathrm{E}-06$ & $0.0 E+00$ \\
\hline Eromoform & $2.6 \mathrm{E}-01$ & $0.0 \mathrm{E}+00$ \\
\hline Bromometh & $7.5 E-07$ & $0.0 \mathrm{E}+00$ \\
\hline Carbondis & $2.0 \mathrm{E}-01$ & $0.0 \mathrm{E}+00$ \\
\hline Chlorometh & $9.5 E-07$ & $0.0 \mathrm{E}+00$ \\
\hline 0 -chtorots & $0.0 \mathrm{E}+00$ & $0.0 \mathrm{E}+00$ \\
\hline Cumene & $0.0 \mathrm{E}+00$ & 6. $4 E=08$ \\
\hline Dibromochloro & $3.6 \mathrm{E}-01$ & $0.0 \mathrm{E}+00$ \\
\hline Dichlorodiflo & 4. $7 \mathbf{E}-02$ & $0.0 \mathrm{E}+00$ \\
\hline Dinbutylphthalat & $2.8 E-18$ & $0.0 \mathrm{E}+00$ \\
\hline 12sisDichloro & $0.0 \mathrm{E}+00$ & $0.0 \mathrm{E}+00$ \\
\hline 12transDichl & 4. $6 \mathbf{E}=01$ & $0.0 \mathrm{E}+00$ \\
\hline 12Dichlprop & $4.2 \mathrm{E}-01$ & $0.0 \mathrm{E}+00$ \\
\hline Ethylbenz & $0.0 \mathrm{E}+00$ & $0.0 \mathrm{E}+00$ \\
\hline
\end{tabular}




\begin{tabular}{|c|c|c|}
\hline Nhexane & $9.4 E-04$ & $1.6 \mathrm{E}-0.3$ \\
\hline Methy l Iso & $4 \cdot 5 E-16$ & $0.0 \mathrm{E}+00$ \\
\hline MethChoride & 8. $1 E-10$ & $0.0 \mathrm{E}+00$ \\
\hline PropGlycol & 2. $8 \mathrm{E}-17$ & $0.0 E+00$ \\
\hline Styrene & $0,0 E+00$ & $0.0 \mathrm{E}+00$ \\
\hline 1112Tetra & $2.0 \mathrm{E}-02$ & $0.0 \mathrm{E}+00$ \\
\hline 1122Tetra & 3. $4 \mathrm{E}-01$ & $0.0 \mathrm{E}+00$ \\
\hline Trichloplo & $1.8 \mathrm{E}-01$ & $0.0 \mathrm{E}+00$ \\
\hline 123Trichlopr & $3.0 \mathrm{E}-01$ & $0.0 \mathrm{E}+0$ \\
\hline Trimethbenz & $0.0 E+00$ & $0.0 \mathrm{E}+00$ \\
\hline 135Trimeth & $0.0 \mathrm{E}+00$ & $0.0 \mathrm{E}+0 \mathrm{C}$ \\
\hline acetophenone & $4.3 E-01$ & $0.0 \mathrm{E}+0 \mathrm{r}$ \\
\hline Ethylehlorid & $9.8 \mathrm{E}-02$ & $0.0 \mathrm{E}+0 \mathrm{C}$ \\
\hline 2Hexanone & $1.2 \mathrm{~B}-07$ & $0.0 E+00$ \\
\hline Metacrylate & $6.1 \mathrm{E}-10$ & $0.0 \mathrm{E}+00$ \\
\hline Methonal & $2.8 \mathrm{E}-17$ & $0.0 \mathrm{~B}+0$ \\
\hline Cyanide & $0.0 E+00$ & $0.0 \mathrm{E}+00$ \\
\hline Dibenz $(a, h)$ & $0.0 \mathrm{E}+00$ & $0.0 \mathrm{E}+00$ \\
\hline 24Dinitrotoluene & $3.4 \mathrm{E}-02$ & $0.0 \mathrm{E}+00$ \\
\hline 26Dini trotoluene & $4.48-02$ & $0.0 \mathrm{E}+0$ \\
\hline Endosulfan & $0.0 E+00$ & $5.7 E-05$ \\
\hline 4Ni trobenzenamin & $0.08+00$ & $1.4 \mathrm{E}-0$ \\
\hline 4Nitrophenol & $0.0 \mathrm{E}+00$ & $0.0 \mathrm{E}+0$ \\
\hline MN1 trosodiphen & $0.0 \mathrm{E}+00$ & $0.0 \mathrm{E}+00$ \\
\hline Xylene & $0.0 \mathbf{E}+00$ & $0.0 \mathrm{E}+00$ \\
\hline 12Dichloro & $0.0 \mathbf{E}+00$ & $0.0 \mathrm{E}+0$ \\
\hline 13Dichloro & $0.0 E+00$ & $8.9 E-2$ \\
\hline 14Dichloro & $0.0 \mathrm{E}+00$ & $0.0 \mathbf{E}+00$ \\
\hline Methylcyclo & $1.8 E-03$ & 1.8E-0 \\
\hline 2Nitrophenol & $0.0 \mathrm{E}+0.0$ & $0.0 \mathrm{E}+0$ \\
\hline EndosulfanII & $0.0 \mathrm{E}+00$ & $5.7 \mathrm{E}-0$ \\
\hline Aldenyde & $0.0 \mathrm{E}+00$ & $3.2 \mathrm{E}-0 \mathrm{x}$ \\
\hline Ketone & $0.0 \mathrm{E}+00$ & $3.2 \mathrm{E}-0$ \\
\hline Chlorobenzene & $0.0 \mathrm{E}+00$ & $0.0 \mathrm{E}+0$ \\
\hline Vinyl Chloride & $0.0 \mathrm{E}+00$ & $0,0 \mathrm{E}+00$ \\
\hline o-cresol & $2.3 \mathrm{E}-01$ & $0.0 \mathrm{E}+00$ \\
\hline mi-cresol & $3.1 \mathrm{E}-01$ & $0.0 \mathrm{E}+0$ \\
\hline p-cresol & 3. $2 \mathrm{E}-01$ & $0,0 \mathrm{E}+0$ \\
\hline
\end{tabular}




$\begin{array}{lll}\text { Hexachlorobenzen } & 0.0 \mathrm{E}+00 & 0.0 \mathrm{E}+00 \\ \text { Hexachloroethane } & 0.0 \mathrm{E}+00 & 0.0 \mathrm{E}+00 \\ \text { Nitrobenzene } & 2.6 \mathrm{E}-01 & 0.0 \mathrm{E}+00 \\ \text { 246-Trichlorphnl } & 0.0 \mathrm{E}+00 & 0.0 \mathrm{E}+00 \\ \text { PYridine } & 2.3 \mathrm{E}-12 & 0.0 \mathrm{E}+00 \\ 24-\mathrm{D} & 8.6 \mathrm{E}-02 & 0.0 \mathrm{E}+00 \\ 245-\mathrm{TP} \text { (Silvex) } & 2.5 \mathrm{E}-02 & 0.0 \mathrm{E}+00 \\ \text { Endrits } & 0.0 \mathrm{E}+0 \mathrm{D} & 3.2 \mathrm{E}-05 \\ \text { Heptachlor } & 0.0 \mathrm{E}+00 & 4.1 \mathrm{E}-21 \\ \text { Heptachlor epoxd } & 0.0 \mathrm{E}+00 & 2.7 \mathrm{E}-05 \\ \text { Lindane } & 0.0 \mathrm{E}+00 & 1.0 \mathrm{E}-03 \\ \text { 124trimethylb } & 0.0 \mathrm{E}+00 & 0.0 \mathrm{E}+00 \\ \text { lhexanol } & 9.1 \mathrm{E}-08 & 0.0 \mathrm{E}+00 \\ \text { 2-Hexanone } & 1.7 \mathrm{E}-01 & 0.0 \mathrm{E}+00 \\ \text { butylbenzene } & 0.0 \mathrm{E}+00 & 0.0 \mathrm{E}+00\end{array}$

CONTAMINANT CONCENTRATIONS IN RIVEF (ING/1)

$\begin{array}{lcc}\quad \text { TIMES } & 1000, & 100000 . \\ \text { Aceraphthene } & 0.0 \mathrm{E}+00 & 0.0 \mathrm{E}+00 \\ \text { Acetone } & 1.1 \mathrm{E}-01 & 0.0 \mathrm{E}+00 \\ \text { Antimony } & 0.0 \mathrm{E}+00 & 7.4 \mathrm{E}-05 \\ \text { Barium } & 0.0 \mathrm{E}+00 & 0.0 \mathrm{E}+00 \\ \text { Eenzene } & 0.0 \mathrm{E}+00 & 0.0 \mathrm{E}+00 \\ \text { Boron } & 0.0 \mathrm{E}+00 & 0.0 \mathrm{E}+00 \\ \text { Carbazole } & 0.0 \mathrm{E}+00 & 7.9 \mathrm{E}-03 \\ \text { Carbontetchl } & 0.0 \mathrm{E}+00 & 0.0 \mathrm{E}+00 \\ \text { Chloroform } & 0.0 \mathrm{E}+00 & 0.0 \mathrm{E}+00 \\ \text { Chromium III } & 0.0 \mathrm{E}+00 & 0.0 \mathrm{E}+00 \\ \text { Dieldrin } & 0.0 \mathrm{E}+00 & \mathbf{8 . 1 \mathrm { E } - 0 2} \\ \text { Isophorone } & 0.0 \mathrm{E}+00 & 0.0 \mathrm{E}+00 \\ \text { Lead } & 0.0 \mathrm{E}+00 & 0.0 \mathrm{E}+00 \\ \text { Manganese } & 0.0 \mathrm{E}+00 & 0.0 \mathrm{E}+00 \\ \text { Naphthalene } & 0.0 \mathrm{E}+00 & 1.4 \mathrm{E}-01 \\ \text { NnitroNpropy1 } & 6.3 \mathrm{E}-06 & 0.0 \mathrm{E}+00 \\ \text { Phenol } & 2.0 \mathrm{E}-03 & 0.0 \mathrm{E}+00 \\ \text { Selenium } & 0.0 \mathrm{E}+00 & 7.9 \mathrm{E}-02\end{array}$




\begin{tabular}{|c|c|c|}
\hline strontium & $0.0 E+00$ & $0.0 \mathrm{E}+00$ \\
\hline Tetrachloroethen & $0.0 E+00$ & $0.0 \mathbf{E}+00$ \\
\hline Tin & $0.0 \mathrm{E}+00$ & $0.0 \mathrm{E}+00$ \\
\hline Toluene & $0.0 \mathrm{E}+00$ & $0 . O E+Q B$ \\
\hline Trich1oroethene & $0.0 \mathrm{E}+0 \mathrm{O}$ & $0.0 \mathrm{E}+0 \mathrm{O}$ \\
\hline $\mathrm{U}-233$ & $0.0 \mathrm{E}+\Theta \theta$ & 4. $5 \mathbf{E}-02$ \\
\hline$U-234$ & $0.0 \mathrm{E}+00$ & $5.2 \mathbf{E}-02$ \\
\hline $\mathrm{U}-235$ & $0.0 E+00$ & $6.9 \mathbf{E}-02$ \\
\hline$v-236$ & $0.0 \mathrm{E}+00$ & $6.9 \mathrm{E}-02$ \\
\hline $\mathrm{U}=238$ & $0.0 \mathrm{E}+00$ & $6.9 E-02$ \\
\hline Vanadium & $0.0 \mathrm{E}+00$ & $0.0 \mathrm{E}+00$ \\
\hline $124 \mathrm{Trichlorb}$ & $0.0 E+00$ & $0.9 \mathrm{E}+0 \mathrm{O}$ \\
\hline Molyböenum & $0.0 \mathrm{E}+00$ & $5.5 E-02$ \\
\hline benzidire & $0 . O E+O B$ & $0.0 \mathrm{E}+00$ \\
\hline Chlordane & $0.0 \mathrm{E}+00$ & $0.0 \mathbf{E}+00$ \\
\hline Methyl PropylB & $0.0 \mathrm{E}+00$ & $0.0 \mathbf{E}+0 \theta$ \\
\hline Methylethyls & $0.0 \mathrm{E}+00$ & $0.0 \mathrm{E}+00$ \\
\hline 12DiMethylB & $0.0 \mathbf{E}+00$ & $0.0 \mathrm{E}+00$ \\
\hline Propyle & $0.0 \mathrm{E}+00$ & $0.0 \mathrm{E}+00$ \\
\hline Alarin & $0.0 E+00$ & $0.0 \mathrm{E}+00$ \\
\hline Aroclor 1221 & C. $G E+00$ & $0.0 \mathbf{E}+00$ \\
\hline Aroclor 1232 & $0.0 \mathrm{E}+00$ & $2.1 E-02$ \\
\hline DDD & $0.0 E+00$ & $0.0 \mathrm{E}+0 \mathrm{O}$ \\
\hline DDE & $0.0 \mathrm{E}+\Theta Q$ & 1. $8 E-64$ \\
\hline Alpha-BHC & $0.0 E+00$ & $3.5 \mathbf{E}-02$ \\
\hline Beta-BHC & $0.0 \mathrm{E}+00$ & $3.5 E-02$ \\
\hline Delta-BHC & $0.0 \mathrm{E}+00$ & $3.5 \mathbf{E}-02$ \\
\hline Accenaphthylene & $0.0 \mathrm{E}+00$ & $7.1 \mathrm{E}-02$ \\
\hline Benzolc Acid & $9.8 E-11$ & $0.0 \mathbf{E}+00$ \\
\hline Dibenzofuran & $0.0 \mathrm{E}+00$ & $0.0 \mathrm{E}+00$ \\
\hline Diethylphth & $4.7 \mathrm{E}+0 \mathrm{O}$ & $0.0 E+00$ \\
\hline 24-Dimethylphe & $0 . Q E+Q \beta$ & $0.0 \mathrm{E}+00$ \\
\hline Dimethylphth & $1.2 \mathbf{E}+01$ & $0.0 \mathrm{E}+00$ \\
\hline 2Methylnaptha & $0.0 \mathrm{E}+00$ & $\theta . \theta \mathbf{E}+\theta \theta$ \\
\hline 2346 Tetrachlor & $0.0 \mathrm{E}+00$ & $\theta . \theta \mathbf{E}+\theta \theta$ \\
\hline Berizyl Alcohol & $1.9 E-04$ & $0.0 \mathrm{E}+00$ \\
\hline Acentondtrile & $6.1 E-16$ & $0.0 \mathrm{E}+00$ \\
\hline Acrolien & $2.2 \mathbf{E}-15$ & $0.0 \mathbf{E}+00$ \\
\hline
\end{tabular}




\begin{tabular}{|c|c|c|}
\hline Acylonitrle & $1,2 \mathrm{E}-14$ & $\begin{array}{l}0.0 E+00 \\
0.0 E+00\end{array}$ \\
\hline Bromodichloro & $2,1 \mathrm{E}-04$ & $0.0 E+00$ \\
\hline Bromoform & $9.0 \mathrm{E}+00$ & $0,0 E+00$ \\
\hline Bromometh & $2.6 \mathrm{E}-05$ & $0,0 \mathrm{E}+00$ \\
\hline CarbonDis & $6.9 E+00$ & $0,0 \mathrm{E}+00$ \\
\hline chlorometh & 3. 3E-0.5 & $0.0 \mathrm{E}+00$ \\
\hline D-Chlorotu & $0.0 E+D 0$ & $0.0 E+00$ \\
\hline Cumene & $0.0 \mathrm{E}+00$ & $2.2 \mathrm{E}-06$ \\
\hline Dibromochloro & $1.2 \mathrm{E}+01$ & $0.0 E+00$ \\
\hline Diehlorodiflo & 1. $6 \mathrm{E}+00$ & $0.0 \mathrm{E}+00$ \\
\hline Dinbutylphthalat & B. $9 E-17$ & $0.0 \mathrm{E}+00$ \\
\hline 12eisDichloro & $0.0 \mathrm{E}+00$ & $0.0 \mathrm{E}+00$ \\
\hline 12transpichl & $1.6 \mathrm{E}+01$ & $0.0 \mathrm{E}+00$ \\
\hline 120ichlprop & 1. $5 E+01$ & $0,0 E+00$ \\
\hline Ethylbenz & $0.0 \mathrm{E}+00$ & $0.0 \mathrm{E}+00$ \\
\hline Nhexane & 3. $3 \mathrm{E}-02$ & $5.6 \mathrm{E}-02$ \\
\hline MethylIso & $1,6 \mathrm{E}-14$ & $0.0 \mathrm{E}+00$ \\
\hline MethChoride & $2 . B E-0 B$ & $0.0 E+\square 0$ \\
\hline Propglyenl & $9.6 \mathrm{E}-16$ & $0.0 \mathrm{E}+00$ \\
\hline styrene & $0.0 \mathrm{E}+00$ & $0.0 \mathrm{E}+00$ \\
\hline 1112 Tetra & $6.9 \mathrm{E}-01$ & $0.0 \mathrm{E}+00$ \\
\hline 1122 Tetra & $1,2 \mathrm{E}+01$ & $0.0 \mathrm{E}+00$ \\
\hline Trichloflo & $6.3 E+00$ & $0.0 E+00$ \\
\hline 123Trichlopr & 1. $0 \mathrm{E}+0 \mathrm{l}$ & $0.0 \mathrm{E}+00$ \\
\hline Trimethbenz & $0.0 E+00$ & $0.0 \mathrm{E}+00$ \\
\hline 135Trimeth & $0.0 \mathrm{E}+00$ & $0.0 \mathrm{E}+00$ \\
\hline acetophenone & 1. $5 \mathrm{E}+01$ & $0.0 \mathrm{E}+00$ \\
\hline Ethylchlorid & $3,4 \mathrm{E}+00$ & $0.0 \mathrm{E}+00$ \\
\hline 2Hexanone & $4.2 \mathrm{E}-06$ & $0.0 \mathrm{E}+00$ \\
\hline Metacrylate & $2.1 \mathrm{E}-0 \mathrm{~B}$ & $0.0 E+00$ \\
\hline Methonal & $9.8 \mathrm{E}-16$ & $0,0 E+00$ \\
\hline Cyanide & $0,0 E+00$ & $0.0 \mathrm{E}+00$ \\
\hline Dibenz $[\mathbf{a}, \mathbf{h}]$ & $0.0 \mathrm{E}+00$ & $0.0 \mathrm{E}+00$ \\
\hline 24Dinitrotoluene & $1.2 \mathrm{E}+00$ & $0.0 \mathrm{E}+00$ \\
\hline 260initrotoluene & $1,6 \mathrm{E}+00$ & $0.0 \mathrm{E}+00$ \\
\hline Endosulfạ & $0.0 \mathrm{E}+00$ & $2.0 \mathrm{E}-03$ \\
\hline $4 \mathrm{Ni}$ trobenzenallin & $0.0 \mathrm{E}+00$ & $4.7 \mathrm{E}-0 \mathrm{~B}$ \\
\hline ANitrophenol & $0.0 \mathrm{E}+00$ & $0.0 E+00$ \\
\hline
\end{tabular}




$\begin{array}{lll}\text { NNitrosodiphen } & 0.0 \mathrm{E}+00 & 0.0 \mathrm{E}+00 \\ \text { Xylene } & 0.0 \mathrm{E}+00 & 0.0 \mathrm{E}+00 \\ \text { 12Dichloro } & 0.0 \mathrm{E}+00 & 0.0 \mathrm{E}+00 \\ \text { 13Dichloro } & 0.0 \mathrm{E}+00 & 3.1 \mathrm{E}-20 \\ \text { 14Dichloro } & 0.0 \mathrm{E}+00 & 0.0 \mathrm{E}+00 \\ \text { Methylcyclo } & 6.2 \mathrm{E}-02 & 6.2 \mathrm{E}-02 \\ \text { 2Nitrophenol } & 0.0 \mathrm{E}+00 & 0.0 \mathrm{E}+00 \\ \text { EndosulfanII } & 0.0 \mathrm{E}+00 & 2.0 \mathrm{E}-03 \\ \text { Aldehyde } & 0.0 \mathrm{E}+00 & 1.1 \mathrm{E}-03 \\ \text { Ketone } & 0.0 \mathrm{E}+00 & 1.1 \mathrm{E}-03 \\ \text { Chlorobenzene } & 0.0 \mathrm{E}+00 & 0.0 \mathrm{E}+00 \\ \text { Vinyl Chlorlde } & 0.0 \mathrm{E}+00 & 0.0 \mathrm{E}+00 \\ \text { o-cresol } & 0.2 \mathrm{E}+00 & 0.0 \mathrm{E}+00 \\ \text { In-cresol } & 1.1 \mathrm{E}+01 & 0.0 \mathrm{E}+00 \\ \text { p-cresol } & 1.1 \mathrm{E}+01 & 0.0 \mathrm{E}+00 \\ \text { 14Dichlorobenzen } & 0.0 \mathrm{E}+00 & 0.0 \mathrm{E}+00 \\ \text { Hexachlorobenzen } & 0.0 \mathrm{E}+00 & 0.0 \mathrm{E}+00 \\ \text { Hexachloroethane } & 0.0 \mathrm{E}+00 & 0.0 \mathrm{E}+00 \\ \text { Nitrobenzene } & 9.2 \mathrm{E}+00 & 0.0 \mathrm{E}+00 \\ \text { 246-Trichlorphn1 } & 0.0 \mathrm{E}+00 & 0.0 \mathrm{E}+00 \\ \text { Pyridine } & 8.2 \mathrm{E}-11 & 0.0 \mathrm{E}+00 \\ \text { 24-D } & 3.0 \mathrm{E}+00 & 0.0 \mathrm{E}+00 \\ \text { 245-TP \{silvex) } & 8.0 \mathrm{E}-01 & 0.0 \mathrm{E}+00 \\ \text { Endrin } & 0.0 \mathrm{E}+00 & 1.1 \mathrm{E}-03 \\ \text { Heptachlor } & 0.0 \mathrm{E}+00 & 1.4 \mathrm{E}-19 \\ \text { Heptachlor epoxd } & 0.0 \mathrm{E}+00 & \mathrm{~B} . \mathrm{BE}-04 \\ \text { Lindane } & 0.0 \mathrm{E}+00 & 3.5 \mathrm{E}-02 \\ \text { l24tritnethylb } & 0.0 \mathrm{E}+00 & 0.0 \mathrm{E}+00 \\ \text { lhexanol } & 3.2 \mathrm{E}-0 \mathrm{0} & 0.0 \mathrm{E}+00 \\ \text { 2hexanone } & 3.2 \mathrm{E}-06 & 0.0 \mathrm{E}+00 \\ \text { butylbenzene } & 0.0 \mathrm{E}+00 & 0.0 \mathrm{E}+00 \\ & & \end{array}$

